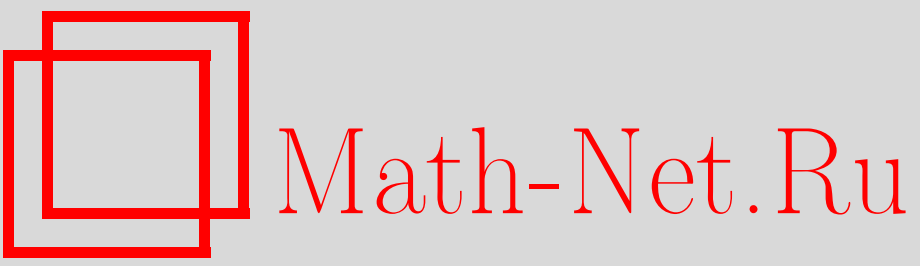

В. Н. Денисов, О поведении решений параболических уравнений при больших значениях времени, УМН, 2005, том 60, выпуск 4, 145-212

DOI: https://doi.org/10.4213/rm1448

Использование Общероссийского математического портала Math-Net.Ru подразумевает, что вы прочитали и согласны с пользовательским соглашением

http://www.mathnet.ru/rus/agreement

Параметры загрузки:

IP : 34.229 .108 .108

26 апреля 2023 г., 14:50:34 


\title{
О ПОВЕДЕНИИ РЕШЕНИЙ ПАРАБОЛИЧЕСКИХ УРАВНЕНИЙ ПРИ БОЛЬШИХ ЗНАЧЕНИЯХ ВРЕМЕНИ
}

\author{
В. Н. ДЕнисов
}

Статья посвящена обзору классических и новых результатов о стабилизации решений (задачи Коши и смешанных задач) для линейных параболических уравнений второго порядка. Приведены доказательства ряда новых результатов о точных достаточных условиях на поведение младших коэффициентов параболического уравнения, которые гарантируют стабилизацию решения задачи Коши для параболического уравнения в классе ограниченных или растущих начальных функций.

Библиография: 93 названия.

\section{СОДЕРЖАНИЕ}

Введение

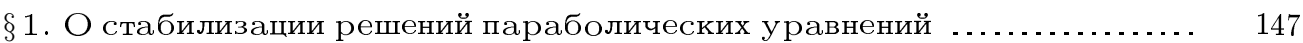

$\S 2$. Некоторые свойства решений эллиптических уравнений $\ldots \ldots \ldots \ldots \ldots . \quad 178$

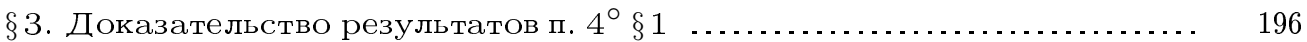

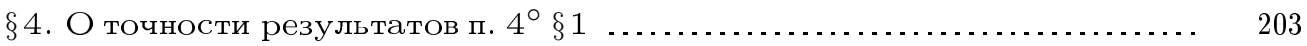

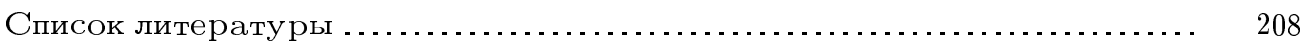

\section{Введение}

Настояшая работа посвяшена обзору ряда вопросов, связанных с нелокальным поведением (при большом времени) решений задачи Коши и смешанных задач для параболических уравнений, в основном второго порядка.

Систематические исследования по качественной теории уравнений параболического типа стали возможными благодаря фундаментальным работам, посвяшенньм обоснованию вопросов разрешимости задачи Коши и смешанных задач для таких уравнений и систем. Из громадного числа работ по корректности постановок упомянутых вьше задач отметим работы С.Н. Бернштейна [1], И.Г. Петровского [2], А.Н. Тихонова [3], М.И. Вишика [4], А. М. Ильина, А. С. Калашникова и О. А. Олейник [5], О. А. Ладыженской [6], В. А. Ильина [7], В. А. Кондратьева [8], а также работы В.П. Михайлова [9] и С.Д. Эйдельмана [10]. Среди зарубежных ученых отметим фундаментальные работы Д. Аронсона и Дж. Серрина [11], Д. Аронсона [12], [13], А. Фридмана [14].

Работа выполнена при финансовой поддержке Российского фонда фундаментальных исследований (грант № 03-01-00138). 
Одними из первых работ по качественному исследованию поведения при большом времени решений параболических уравнений являются работы А.Н. Тихонова [15] и А. Н. Колмогорова, И. Г. Петровского и Н. С. Пискунова [16]. В работе [15] изучалось поведение при $t \rightarrow \infty$ решения первой краевой задачи для уравнения теплопроводности, а в работе [16] изучалось поведение при $t \rightarrow \infty$ решения задачи Коши для почти линейного параболического уравнения второго порядка с одной пространственной переменной. Обзор по обширной тематике, инициированньй работой [16], содержится, например, в [17]. В настоящей работе мы рассмотрим случай линейных уравнений параболического типа. Будем говорить, что решение $u(x, t)$ задачи Коши или смешанной задачи для параболического уравнения стабилизируется в точке $x \in \mathbb{R}^{N}$, если в этой точке существует предел

$$
\lim _{t \rightarrow \infty} u(x, t)
$$

Если предел (1) сушествует равномерно относительно $x$ на компакте $K$ в $\mathbb{R}^{N}$, то говорят о равномерной на $K$ стабилизации. Если предел (1) существует равномерно относительно $x$ во всем $\mathbb{R}^{N}$, то говорят, что $u(x, t)$ равномерно в $\mathbb{R}^{N}$ стабилизируется. Отметим, что исследование поведения при большом времени решений задачи Коши и смешанных задач (даже в случае уравнения теплопроводности) потребовало значительных усилий, и ответы на вопросы о необходимых и достаточных условиях стабилизации (даже задачи Коши для уравнения теплопроводности) получены сравнительно недавно [18]. Отметим особо работы [19] В. В. Жикова и [20] С. Л. Каменомостской (S. Kamin), в которых получены критерии поточечной (равномерной в $\mathbb{R}^{N}$ ) стабилизации решения задачи Коши для параболического уравнения второго порядка с дивергентной эллиптической частью без младших членов в случае, когда начальная функция $u(x, 0)$ является ограниченной (или, более обще, $\left.u(x, 0) \in L^{\infty}\left(\mathbb{R}^{N}\right)\right)$.

Одним из первых вопросы о достаточных условиях стабилизации решения задачи Коши и смешанных задач изучил М. Кржижанский [21]-[23], которьй применил метод, основанный на принципе максимума и построении соответствующих вспомогательных функций сравнения (барьеров). В работе А. М. Ильина [24] изучены достаточные условия на младшие коэффициенты параболического уравнения второго порядка, при выполнении которых решение задачи Коши стабилизируется к нулю при любой ограниченной начальной функции, стремящейся к нулю при $|x| \rightarrow \infty$. При этом установлено, что достаточные условия стабилизащии в [24] являются неулучшаемыми.

Обзор работ по стабилизации решения задачи Коши и смешанных задач, вьполненных до 1961 г., содержится в $\S 12$ статьи [5]. В п. $4^{\circ} \oint 1$ настояшей работы будет приведен ряд результатов, которые являются развитием и уточнением результатов работ [5], [24].

Для случая, когда область содержит бесконечность, поведение решения первой и второй смешанной задачи (задачи Неймана) было исследовано в работах [25]-[28] для параболического уравнения второго порядка с дивергентной эллиптической частью и без младших членов. Отметим, что в вопросах стабилизации решения задачи Коши и смешанных задач для параболических уравнений большую роль играет наличие (или отсутствие) в соответствуюшем параболическом уравнении младших членов. В связи с этим обстоятельством развитие теории стабилизации решений параболических уравнений идет в основном по двум направлениям, в первом из которых изучаются условия на начальную функцию и старшие коэффициенты уравнения (и еше на об- 
ласть, в случае смешанных задач), когда младшие коэффициенты уравнения не влияют на стабилизацию, а во втором направлении изучаются условия на младшие коэффициенты параболического уравнения, при которых решение стабилизируется при произвольной функции $u(x, 0)$ из некоторого класса единственности соответствующей задачи.

Отметим также, что в большинстве работ по стабилизации решений параболических уравнений накладьвается жесткое требование ограниченности (или даже суммируемости по Лебегу) начальной функции в соответствующей области и требование ограниченности коэффициентов параболического уравнения. Вопросы о необходимых и достаточных условиях поточечной стабилизации решения задачи Коши (даже для уравнения теплопроводности) в случае растущих начальных функций (из класса А.Н. Тихонова [3] или степенного класса) были решены в работе [29] (см. также [30]). Влияние младших коэффициентов параболического уравнения на стабилизацию решения задачи Коши и смешанных задач в случае ограниченных начальных функций исследовано в работах Р.З. Хасьминского [31] и А.М. Ильина и Р.З. Хасьминского $[32]$.

Точные достаточные условия на младшие коэффициенты параболического уравнения, гарантируюшие стабилизацию решения задачи Коши с любой начальной функщией $u_{0}(x)$, растущей на бесконечности не быстрее некоторой фиксированной степени $|x|^{m}, m>0$, были получены в работах [33]-[35].

В настоящее время отсутствуют обзорные работы, посвяшенные последовательному изложению всех основных фактов теории стабилизации решений параболических уравнений.

Обзор по работам, посвященным влиянию характеристик области и ее гранищы на стабилизацию решений смешанных задач (в основном задачи Неймана) для параболических уравнений без младших членов, можно найти в работах [36], [37]. Обзору работ по стабилизащии решения задачи Коши для параболического уравнения без младших членов и с ограниченными или растушими начальными функциями посвящена работа [38].

Настоящую работу мы посвяшаем обзору работ по стабилизации решений параболических уравнений, вьполненных за последние десятилетия, которые находятся в области наших интересов и развивают результаты основателей и первых творцов этой замечательной ветви теории уравнений в частных производных.

В статье принята сквозная нумерация в каждом параграфе, а при ссылке на формулу $\left(N_{1}\right)$ из $\S N_{2}$ мы пишем $\left(N_{1}, N_{2}\right)$.

\section{§ 1. О стабилизации решений параболических уравнений}

$\mathbf{1}^{\circ}$. Определения и исходные факты. Приведем список применяемых далее обозначений:

$$
\begin{gathered}
x=\left(x_{1}, \ldots, x_{N}\right) \in \mathbb{R}^{N}, \quad \mathbb{R}_{+}^{1}=\{t: t>0\}, \quad \overline{\mathbb{R}}_{+}^{1}=\{t: t \geqslant 0\}, \\
\mathbb{R}_{+}^{N+1}=\left\{x, t: x \in \mathbb{R}^{N}, t>0\right\}, \quad \overline{\mathbb{R}}_{+}^{N+1}=\left\{x, t: x \in \mathbb{R}^{N}, t \geqslant 0\right\},
\end{gathered}
$$

$H_{\left(t_{1}, t_{2}\right]}=\left\{x, t: x \in \mathbb{R}^{N}, t_{1}<t \leqslant t_{2}\right\}-$ слой в $\mathbb{R}_{+}^{N+1}$, в частности, $H=H_{(0, T]}$, $\Omega$ - ограниченная область в $\mathbb{R}^{N}, B_{R}^{x_{0}}=\left\{x \in \mathbb{R}^{N}:\left|x-x_{0}\right|<R\right\}$ - открытьй шар с центром в $x=x_{0}$ радиуса $R, \bar{B}_{R}^{x_{0}}=\left\{x \in \mathbb{R}^{N}:\left|x-x_{0}\right| \leqslant R\right\}$, в частности, 
$B_{R} \equiv B_{R}^{0}$; объем шара $B_{R}^{x_{0}}$ в $\mathbb{R}^{N}$ вычисляется по формуле $\left|B_{R}^{x_{0}}\right|=R^{N} \omega_{N} N^{-1}$, где $\omega_{N}=2 \pi^{N / 2}[\Gamma(N / 2)]^{-1}-$ площадь сферы радиуса 1 в $\mathbb{R}^{N}, \Gamma(\nu)$ - интеграл Эйлера второго рода; $S_{\lambda_{1}}^{x_{0}}=\left\{x \in \mathbb{R}^{N}: \frac{\lambda_{1}}{2}<\left|x-x_{0}\right|<2 \lambda_{1}\right\}$ - открытьй шаровой слой в $\mathbb{R}^{N}$, в частности,

$$
S_{\lambda_{1}} \equiv S_{\lambda_{1}}^{0}, \quad a(x)=a_{i k}(x) \quad(i, k=1, \ldots, N),
$$

$a(x, t)=a_{i k}(x, t)(i, k=1, \ldots, N)$ - квадратные матрицы размера $N \times N$ с вещественными коэффициентами,

$$
(a(x) \xi, \xi)=\sum_{i, k=1}^{N} a_{i k}(x) \xi_{i} \xi_{k}, \quad(a(x, t) \xi, \xi)=\sum_{i, k=1}^{N} a_{i k}(x, t) \xi_{i} \xi_{k}
$$

- квадратичные формы, порождаемые матрицами $a(x)$ и $a(x, t)$ соответственно. Всегда будет предполагаться симметричность матриц $a(x)$ и $a(x, t)$, т.е.

$$
a_{i k}(x)=a_{k i}(x), \quad a_{i k}(x, t)=a_{k i}(x, t) \quad(i, k=1, \ldots, N) ;
$$

$\nabla V=\left(V_{x_{1}}, \ldots, V_{x_{N}}\right)=\operatorname{grad} V(x)-$ градиент скалярной функщии $V(x)$,

$$
\begin{gathered}
V_{x_{i}} \equiv \frac{\partial}{\partial x_{i}} V \quad(i=1, \ldots, N) \\
V_{x_{i} x_{k}}=\frac{\partial^{2} V}{\partial x_{i} \partial x_{k}} \quad(i, k=1, \ldots, N), \\
\operatorname{div} V=\sum_{i=1}^{N} V_{x_{i}}
\end{gathered}
$$

Пусть $Q$ - область в $\mathbb{R}^{N+1}$, под пространством $W_{2}^{1,0}(Q)$ будем понимать [12], [39] пополнение множества финитных функций $C_{0}^{\infty}\left(\mathbb{R}^{N+1}\right)$ по норме

$$
\|f\|_{W_{2}^{1,0}(Q)}=\left[\int_{Q}\left(f^{2}(x, t)+(\nabla f, \nabla f)\right) \mathrm{d} x \mathrm{~d} t\right]^{1 / 2},
$$

где $(\nabla f, \nabla f)$ - скалярньй квадрат вектора $\nabla f,(x, y)$ - скалярное произведение в $\mathbb{R}^{N}$.

Аналогично, пространство $W_{2}^{1,1}(Q)$ есть пополнение множества финитных функций $C_{0}^{\infty}\left(\mathbb{R}^{N+1}\right)$ по норме [39]

$$
\|f\|_{W_{2}^{1,1}(Q)}=\left[\int_{Q}\left(f^{2}(x, t)+f_{t}^{2}(x, t)+(\nabla f, \nabla f)\right) \mathrm{d} x \mathrm{~d} t\right]^{1 / 2} .
$$

Пусть $L(x)$ - оператор вида

$$
L(x)=\sum_{i, k=1}^{N} \frac{\partial}{\partial x_{i}}\left(a_{i k}(x) \frac{\partial}{\partial x_{k}}\right), \quad a_{i k}(x)=a_{k i}(x),
$$

где $x=\left(x_{1}, \ldots, x_{N}\right) \in \mathbb{R}^{N}, a_{i k}(x)$ - измеримые и ограниченные функции в $\mathbb{R}^{N}$ и вьполняется условие

$$
\lambda^{-1}|\xi|^{2} \leqslant(a(x) \xi, \xi) \leqslant \lambda|\xi|^{2}, \quad \lambda>0, \quad|\xi|^{2}=\sum_{i=1}^{N} \xi_{i}^{2}
$$


Будем рассматривать также оператор

$$
\mathscr{L}(x)=\sum_{i, k=1}^{N} a_{i k}(x) \frac{\partial^{2}}{\partial x_{i} \partial x_{k}},
$$

где на симметрическую матрицу $a_{i k}(x)$ наложены те же условия (2).

Если коэффициенты в (1) или в (3) зависят еше от $t$, т.е. $a_{i k}(x, t)$, то будем писать в (1) и в $(3) L(x, t), \mathscr{L}(x, t)$, где $t \geqslant 0$, вместо $L(x)$ или $\mathscr{L}(x)$.

$\mathrm{B} \mathbb{R}_{+}^{N+1}$ рассмотрим уравнение

$$
\sum_{i, k=1}^{N}\left(a_{i k}(x, t) u_{x_{i}}\right)_{x_{k}}+\sum_{i=1}^{N} b_{i}(x, t) u_{x_{i}}+c(x, t) u-u_{t}=0
$$

или, кратко,

$$
L(x, t) u+(b(x, t), \nabla u)+c(x, t) u-u_{t}=0,
$$

удовлетворяющее начальному условию

$$
u(x, 0)=u_{0}(x), \quad x \in \mathbb{R}^{N}, \quad\left|u_{0}(x)\right| \leqslant M .
$$

Всегда будем предполагать ${ }^{1}$, что $\lambda^{-1}|\xi|^{2} \leqslant(a(x, t) \xi, \xi) \leqslant \lambda|\xi|^{2}(\lambda>0)$,

$$
\left|b_{i}(x, t)\right| \leqslant M, \quad|c(x, t)| \leqslant M, \quad c(x, t) \leqslant 0 \quad \text { в } \quad \mathbb{R}_{+}^{N+1} .
$$

Под обобщенным решением задачи Коши $(4),(5)$ в $\mathbb{R}_{+}^{N+1}$ будем понимать (см., например, [12], [39]) такую функцию $u(x, t)$, которая при всех $R>0, T>0$ принадлежит пространству $W_{2}^{1,0}\left(B_{R} \times(0, T)\right)$ и при каждом $T>0$ удовлетворяет интегральному тождеству

$$
\int_{\mathbb{R}_{+}^{N+1}}\left[(a \nabla u, \nabla \eta)-\eta((b, \nabla u)+c u)-\eta_{t} u\right] \mathrm{d} x \mathrm{~d} t=\int_{\mathbb{R}^{N}} u_{0}(x) \eta(x, 0) \mathrm{d} x
$$

при всех пробных функциях $\eta(x, t) \in C_{0}^{\infty}\left(\mathbb{R}^{N+1}\right)$, носитель которых лежит в полупространстве $\left\{x, t: x \in \mathbb{R}^{N}, t<T\right\}$. Известно (см., например, [12], [39]), что если функция $u_{0}(x)$ является ограниченной (точнее, если $u_{0}(x) \in L^{\infty}\left(\mathbb{R}^{n}\right)$ ) и если ее норму обозначить через $\left\|u_{0}\right\|_{L^{\infty}\left(\mathbb{R}^{N}\right)}$, то решение задачи Коши (4), (5) существует, ограничено и такое решение единственно и принадлежит $C\left(\mathbb{R}_{+}^{N+1}\right)$ и

$$
\|u(\cdot, t)\|_{L^{\infty}\left(\mathbb{R}^{N}\right)} \leqslant C\left\|u_{0}\right\|_{L^{\infty}\left(\mathbb{R}^{N}\right)} .
$$

Рассмотрим случай недивергентного уравнения, т.е. когда параболическое уравнение имеет вид

$$
\sum_{i, k=1}^{N} a_{i k}(x, t) u_{x_{i} x_{k}}+\sum_{i=1}^{N} b_{i}(x, t) u_{x_{i}}+c(x, t) u-u_{t}=0 \quad \text { в } \mathbb{R}_{+}^{N+1},
$$

\footnotetext{
${ }^{1}$ Более слабые условия см. в [12].
} 
или, кратко,

$$
\mathscr{L}(x, t) u+(b(x, t), \nabla u)+c(x, t) u-u_{t}=\begin{array}{lll}
0 & \text { в } \mathbb{R}_{+}^{N+1},
\end{array}
$$

где $u(x, t)$ - классическое решение (т.е. $u(x, t)$ непрерьвна в $\overline{\mathbb{R}}_{+}^{N+1}$ и имеет непрерьвные производные, входящие в (8)), удовлетворяющее уравнению (8) и начальному условию

$$
u(x, 0)=u_{0}(x), \quad\left|u_{0}(x)\right| \leqslant M, \quad x \in \mathbb{R}^{N} .
$$

В дальнейшем будем предполагать, что выполнены следующие условия (см., например, [14]) на коэффициенты уравнения (9):

1) оператор $\mathscr{L}(x, t)$ является параболическим в $H_{(0, T]}$ для любого $T>0$, т.е. для каждой точки $(x, t) \in \mathbb{R}_{+}^{N+1}$ выполнены неравенства $(2)$;

$2)$ коэффициенты уравнения (9) - непрерьвные в $H_{(0, T]}$ функции, удовлетворяющие равномерному условию Гёльдера в $H_{(0, T]}$;

3) в $H_{(0, T]}$ вьполнены неравенства (6),

и будем считать, что функция $u(x, t)$ в $(9)$ имеет в $\mathbb{R}_{+}^{N+1}$ непрерывные производные по переменным $x$ до второго порядка включительно и непрерьвные производные первого порядка по $t$.

В работах [5], [14] установлено, что если $\mathscr{L}(x, t)$ - параболический оператор с непрерьвньми коэффищиентами и выполнены условия

$$
\left|a_{i k}(x, t)\right| \leqslant M, \quad\left|b_{i}(x, t)\right| \leqslant M, \quad c(x, t) \leqslant M\left(1+|x|^{2}\right)
$$

то сушествует не более одного решения задачи Коши $(9),(10)$ в $H_{(0, T]}$, удовлетворяюшего условию

$$
|u(x, t)| \leqslant B \exp \left\{\beta|x|^{2}\right\}
$$

с некоторыми постоянньми $\beta>0$ и $B>0$.

Пусть $u(x, t)$ - решение задачи Коши (4), (5) (или (9), (10)). Будем говорить, что решение $u(x, t)$ стабилизируется в точке $x \in \mathbb{R}^{N}$, если сушествует предел

$$
\lim _{t \rightarrow \infty} u(x, t)=V(x)
$$

Если предел (12) сушествует равномерно по $x \in \mathbb{R}^{N}$, то будем говорить, что решение $u(x, t)$ стабилизируется равномерно по $x \in \mathbb{R}^{N}$; если предел (12) сушествует равномерно по $x$ на $K$, где $K$ - произвольный компакт $K \subset \mathbb{R}^{N}$, то будем говорить, что решение $u(x, t)$ стабилизируется равномерно по $x$ на любом компакте $K$ в $\mathbb{R}^{N}$.

$\mathrm{B} \mathbb{R}^{N}$ рассмотрим эллиптическое уравнение

$$
\sum_{i, k=1}^{N}\left(a_{i k}(x) V_{x_{k}}\right)_{x_{i}}+\sum_{i=1}^{N} b_{i}(x) V_{x_{i}}+c(x) V=0
$$

или, кратко,

$$
L(x) V+(b(x), \nabla V)+c(x) V=0 \quad \text { в } \quad \mathbb{R}^{N} .
$$

Будем считать, что коэффищиенты уравнения (13) - ограниченные и измеримые функции в $\mathbb{R}^{N}, a_{i k}(x)=a_{k i}(x)(i, k=1, \ldots, N)$ и выполнено условие эллиптичности (2). Под локальным решением уравнения (13) в $\mathbb{R}^{N}$ будем понимать решение 
в стандартном определении [40]. Именно, функция $V(x)$ назьвается обобщенньм решением уравнения (13) в области $\Omega \subseteq \mathbb{R}^{N}$, если $V(x) \in W_{2}^{1}\left(\Omega^{1}\right) \cap L^{\infty}\left(\Omega^{1}\right)$, где $\Omega^{1}-$ произвольная ограниченная подобласть $\Omega^{1} \subset \Omega$ такая, что $\overline{\Omega^{1}} \subset \Omega$, и справедливо интегральное тождество

$$
\int_{\Omega}[-(a \nabla V, \nabla \varphi)+\varphi((b, \nabla V)+c V)] \mathrm{d} x=0
$$

при любой пробной функщии $\varphi(x) \in C_{0}^{\infty}(\Omega)$, имеющей компактньй носитель в $\Omega$.

ЗАмЕчАнИЕ 1. Если коэффициенты уравнения (4) не зависят от $t$, т.е. уравнение имеет вид

$$
L(x) u+(b(x), \nabla u)+c(x) u-u_{t}=0 \quad \text { в } \mathbb{R}_{+}^{N+1},
$$

и решение задачи Коши (15), (5) стабилизируется равномерно по $x$ в некоторой области $\Omega \subseteq \mathbb{R}^{N}$, то легко видеть, что функция $V(x)$, определяемая в (12), удовлетворяет уравнению

$$
L(x) V+(b(x), \nabla V)+c(x) V=0, \quad x \in \Omega \subseteq \mathbb{R}^{N},
$$

в смысле интегрального тождества (14).

Путем замены $W(x, t)=u(x, t)-V(x)$, где $u(x, t)-$ решение задачи Коши $(15)$, (5), а $V(x)$ - решение уравнения (13), мы сведем вопрос о существовании предела (12) к случаю, когда предельная функция в (12) является нулевой, т.е.

$$
\lim _{t \rightarrow \infty} W(x, t)=0 .
$$

Аналогичное замечание можно сделать и для случая, когда коэффициенты уравнения (4) равномерно по $x \in \mathbb{R}^{N}$ стремятся при $t \rightarrow \infty$ к предельньм функциям, зависящим от $x$.

ЗАмЕчАниЕ 2. Если коэффициенты уравнения (9) имеют предельные функции

$$
\lim _{t \rightarrow \infty} a_{i k}(x, t)=a_{i k}(x), \quad \lim _{t \rightarrow \infty} b_{i}(x, t)=b_{i}(x), \quad \lim _{t \rightarrow \infty} c(x, t)=c(x)
$$

равномерно по $x$ в некоторой области $\Omega \subseteq \mathbb{R}^{N}$ и соответствующее эллиптическое уравнение

$$
\mathscr{L}(x) V+(b(x), \nabla V)+c(x) V=0
$$

имеет классическое решение $V(x)$, определяемое для всех $x$ из области $\Omega\left(\right.$ в $\left.\mathbb{R}^{N}\right)$, и если это решение $V(x)$ ограничено вместе со своими производньми, входящими в уравнение, то путем замены $W(x, t)=u(x, t)-V(x)$ мы сведем вопрос о сушествовании предела $(12)$ в $\Omega\left(\right.$ в $\left.\mathbb{R}^{N}\right)$ к вопросу о существовании нулевого предела $(17)$ в $\Omega\left(\right.$ в $\left.\mathbb{R}^{N}\right)[5]$.

$2^{\circ}$. О стабилизации решений параболических уравнений с младшими членами. Недивергентный случай. В 1938 г. А.Н. Тихонов доказал [15], что если $\Omega$ - ограниченная область в $\mathbb{R}^{N}, \Omega \subset \mathbb{R}^{N}, G=\Omega \times(0, \infty)$ - прямой полуцилиндр с основанием $\Omega$ и $u(x, t)$ - решение первой смешанной задачи для уравнения теплопроводности в цилиндре $G$ :

$$
\begin{gathered}
\Delta u-u_{t}=0, \quad(x, t) \in G=\Omega \times(0, \infty), \\
\left.u\right|_{\partial \Omega}=\varphi(x), \quad t>0, \quad u(x, 0)=0,
\end{gathered}
$$


то решение $u(x, t)$ этой задачи стабилизируется в $G$ равномерно по $x \in \Omega$ к функции $V(x)$ :

$$
\lim _{t \rightarrow \infty} u(x, t)=V(x),
$$

которая является решением уравнения Лапласа в области $\Omega$ и принимает на границе $\partial \Omega$ значения функции $\varphi(x)$, т.е.

$$
\Delta V=0 \quad \text { в } \Omega,\left.\quad V\right|_{\partial \Omega}=\varphi(x) .
$$

В работе В. Фулкса [41] сформулированньй выше результат А.Н. Тихонова был обобщен следующим образом.

Пусть $u(x, t)$ - решение уравнения теплопроводности в полуцилиндре $G$. Пусть при $t=0 u(x, 0)=0$, и пусть $\psi(x, t) \geqslant 0$ - непрерывная граничная функция $\left.u\right|_{\partial \Omega}=\psi(x, t)$, $t>0$, где $\psi(x, t)$ не убьвает по $t$ и сходится при $t \rightarrow+\infty$ к непрерьвной функции $\varphi(x)=\lim _{t \rightarrow \infty} \psi(x, t), x \in \partial \Omega$. Тогда решение $u(x, t)$ положительно, не убьвает по $t$ и стабилизируется равномерно по $x \in \Omega$ к функции $V(x)$, являюшейся решением задачи Дирихле (18) для уравнения Лапласа в области $\Omega$.

В работе Р. Нарасимхана [42] исследован вопрос о достаточных условиях асимптотической устойчивости при $t \rightarrow \infty$ (обобщающей понятие асимптотической устойчивости, исследованной А. М. Ляпуновым для обыкновенных дифференциальных уравнений) решений первой краевой задачи для параболических систем второго порядка с коэффициентами, зависяшими только от $x$. Асимптотическая устойчивость решений краевых задач для общих параболических уравнений второго порядка была также исследована в работе [43]. Уравнения в [42] и [43] содержат слабые нелинейности в правых частях.

В работе М. Кржижанского [21] доказано, что регулярное решение однородного параболического уравнения

$$
a(x, t) u_{x x}+b(x, t) u_{x}+c(x, t) u-u_{t}=0, \quad 0<x<1, \quad t>0,
$$

где $a(x, t) \geqslant a_{0}>0, c(x, t) \leqslant 0$ и коэффициенты уравнения (19) непрерывны внутри полосы $\{x, t: 0 \leqslant x \leqslant 1, t \geqslant 0\}$, стабилизируется к нулю равномерно по $x \in[0,1]$, если сушествуют пределы

$$
\lim _{t \rightarrow \infty} u(0, t)=\lim _{t \rightarrow \infty} u(1, t)=0 .
$$

Аналогичный результат был установлен М. Кржижанским в [22] и для случая, когда уравнение (19) рассматривается в четверть-пространстве $\{x, t: 0 \leqslant x<\infty, t>0\}$.

А. Фридман доказал [14], [44], [45] теоремы о равномерной стабилизации к нулю при $t \rightarrow+\infty$ решений краевых задач для параболического уравнения вида (9) с правой частью $f(x, t)$, рассматриваемых или в полуцилиндре, или в расширяюшейся области и при условии, что граничные функции стремятся к нулю при $t \rightarrow \infty$. Там же сфформулированы и доказаны теоремы, обобщаюшие сформулированный выше результат А.Н. Тихонова [15] и В. Фулкса [41] на случай неоднородного параболического уравнения.

В $[5 ; \S 12]$ дан обзор результатов о стабилизации решений задачи Коши и смешанных задач для параболических уравнений. Так как мы далее обобшим ряд результатов из [5], то для удобства читателя мы приведем ряд результатов из [5; 12$]$.

Предполагается, что коэффициенты уравнения (9) ограничены и коэффициент $c(x, t)$ удовлетворяет неравенству (6) и что рассматриваемые решения и $u_{0}(x)$ ограничены в $\overline{\mathbb{R}}_{+}^{N+1}$. 
ТеОРема 1 [5; $\S 12]$. Пусть $и(x, t)$ является решением задачи Коши (9), (10) или решением уравнения (9) в иилиндре $G=\Omega \times(0, \infty)$, где $\Omega$ - ограниченная область в $\mathbb{R}^{N}$, удовлетворяющим начальному условию $u(x, 0)=u_{0}(x), x \in \Omega, u$ одному из граничных условий

$$
\left.u\right|_{\partial \Omega}=0, \quad t>0
$$

uлu

$$
\left.l(u) \equiv\left(\frac{\partial u}{\partial \nu}+a u\right)\right|_{\partial \Omega}=0, \quad t>0,
$$

где $\partial \Omega \times(0, \infty)$ - боковая поверхность иилиндра $G, a(x) \leqslant 0, \nu$ - направление в $\mathbb{R}^{N}$, составляющее острый угол с внутренней нормалью к гранище области $\Omega$.

Пусть

$$
c(x, t) \leqslant-C_{0}<0
$$

где $C_{0}-$ постоянная. Тогда

$$
\lim _{t \rightarrow \infty} u(x, t)=0
$$

равномерно по $x \in \Omega$.

В теореме $2 \S 12$ работы [5] доказано, что для случая первой краевой задачи условие $(21)$ можно отбросить. Пусть функция $u(x, t)$ удовлетворяет в цилиндре $G=$ $\Omega \times(0, \infty)$ уравнению $(9)\left(\Omega\right.$ - ограниченная область в $\left.\mathbb{R}^{N}\right)$, начальному условию $u(x, 0)=u_{0}(x), x \in \Omega$, и пусть $\left.u\right|_{\partial \Omega}=0$ при $t>0$, тогда сушествует предел $(22)$, равномерный по $x \in \Omega$.

Если дополнительно потребовать, чтобы выполнялось условие

$$
a(x, t) \leqslant-a_{0}<0 \text { для } x \in \partial \Omega, t>0,
$$

тогда, как доказано в теореме $3 \S 12$ [5], решение уравнения (9), удовлетворяющее начальному условию $u(x, 0)=u_{0}(x), x \in \Omega$, и на боковой поверхности цилиндра краевому условию

$$
\left.\left(\frac{\partial u}{\partial \nu}+a(x, t) u\right)\right|_{\partial \Omega}=0, \quad t>0,
$$

имеет предел (22) равномерно по $x \in \Omega$.

Таким образом, в теореме 1 можно отбросить условие $(21)$ на коэффищиент $c(x, t)$, если вьполнено условие (23). При доказательстве указанных теорем 1-3 из [5; 12$]$ применяется приншип максимума в ограниченной области для смешанных задач и принцип максимума для полосы $H(0, T]$ в случае задачи Коши. Соответствуюшие варианты принципа максимума доказаны в $[5 ; \S 1]$.

ЗАмечАнИЕ 1 . В теоремах 2 и $3[5 ; \S 12]$ условия удовлетворения $u(x, t)$ уравнению $(9)$ в цилиндре $G=\Omega \times(0, \infty)$ и соответственно условиям (20) или $(24)$ можно заменить на условия удовлетворения функцией $u(x, t)$ следуюшим неравенствам (теорема 2):

$$
\begin{gathered}
\mathscr{L}(x, t) u+(b(x, t), \nabla u)+c(x, t) u-u_{t} \leqslant 0 \text { в } G, \\
\left.u\right|_{\partial \Omega} \geqslant 0, \quad t>0,
\end{gathered}
$$


или неравенствам (теорема 3)

$$
\begin{gathered}
\mathscr{L}(x, t) u+(b(x, t), \nabla u)+c(x, t) u-u_{t} \leqslant 0 \quad \text { в } G \\
\frac{\partial u}{\partial \nu}+\left.a(x, t) u\right|_{\partial \Omega} \leqslant 0, \quad t>0
\end{gathered}
$$

тогда предел (22) в утверждениях этих теорем следует заменить на следуюший предел:

$$
\lim _{t \rightarrow \infty}\left[\inf _{x} u(x, t)\right] \geqslant 0
$$

Это замечание основано на том факте, что принцип максимума в цилиндре $G=$ $\Omega \times(0, \infty)$ справедлив не только для решений $u(x, t)$ уравнения $(9)$, но и для функций $u(x, t)$, которые являются суперпараболическими функциями, т.е. удовлетворяют неравенствам (25) или (26).

В работе [46] установлен принщип максимума для суперпараболических функций, понимаемых и в слабом смысле, т.е. когда соответствуюшие неравенства трактуются в интегральном смысле.

В теореме 4 (см. [ $5 ; \S 12])$ доказано, что если $u(x, t)$ - решение задачи Коши $(9),(10)$ с ограниченной начальной функцией $u_{0}(x)$ и если сушествует такая положительная в $\mathbb{R}^{N}$ функция $V(x)$, что

$$
\begin{aligned}
\mathcal{L}(x, t) V+(b(x, t), & \nabla V)+c(x, t) V \leqslant 0, \quad(x, t) \in \mathbb{R}_{+}^{N+1}, \\
& \lim _{|x| \rightarrow \infty} V(x)=+\infty
\end{aligned}
$$

то $\lim _{t \rightarrow \infty} u(x, t)=0$ равномерно по $x$ на любом компакте $K$ в $\mathbb{R}^{N}$.

Утверждение теоремы 4 носит условный характер, ибо требуется указать условия на коэффициенты уравнения (9), которые гарантировали бы сушествование функции $V(x)>0$, удовлетворяюшей соотношениям $(27)$ и $(28)$.

С этой целью была установлена теорема 5 (см. $[5 ; \S 12]$ ), которая утверждает, что если $u(x, t)$ - ограниченное решение задачи Коши $(9),(10)$ с ограниченной начальной функцией, коэффициент $c(x, t)$ удовлетворяет неравенству $c(x, t) \leqslant-C_{0}<0$ для всех $x \in G_{1}$, где $G_{1}$ - ограниченная область в $\mathbb{R}^{N}$, и существует положительная функция $V(x), x \in \mathbb{R}^{N}$, удовлетворяюшая соотношению (27) при $|x| \geqslant R, t>0$ и такая, что вьполнено условие $(28)$, то $\lim _{t \rightarrow \infty} u(x, t)=0$ равномерно по $x$ на любом компакте $K$ в $\mathbb{R}^{N}$.

Легко видеть, что достаточньм условием существования функции $V(x)$ в теореме 5 с требуемьми свойствами является расходимость следуюшего интеграла:

$$
\lim _{R \rightarrow \infty} \int_{R}^{\infty} r \exp \left(-\int_{r_{0}}^{r} \frac{q(y)}{y} \mathrm{~d} y\right) \mathrm{d} r=+\infty,
$$

где

$$
q(y)=\sup _{\substack{|x|=y \\ t>0}} \frac{\sum_{i=1}^{N} a_{i i}(x, t)+b_{i}(x, t) x_{i}}{\sum_{i, k=1}^{N} a_{i k}(x, t)\left(x_{i} x_{k} / y^{2}\right)}
$$

при этом в качестве функции $V(|x|)$ в теореме 5 следует взять функцию

$$
V(|x|)=\int_{R}^{|x|} r \exp \left\{-\int_{r_{0}}^{r} \frac{q(y)}{y} \mathrm{~d} y\right\} \mathrm{d} r .
$$


Известно, что решение задачи Коши для уравнения теплопроводности $\Delta u-u_{t}=0$ с начальной функцией $u(x, 0)=u_{0}(x)$, которая стремится к нулю при $|x| \rightarrow \infty$, само стремится к нулю при $t \rightarrow \infty$ (см. [24]).

Тот же результат имеет место и для уравнений с постоянными коэффициентами

$$
\sum_{i, k=1}^{N} a_{i k} u_{x_{i} x_{k}}+\sum_{i=1}^{N} b_{i} u_{x_{i}}+c u-u_{t}=0
$$

при условии, что $c \leqslant 0$. Это легко следует из явной формулы для решения. Однако подобное утверждение о существовании предела $\lim _{t \rightarrow \infty} u(x, t)=0$ решения задачи Коши (9), (10) уже не имеет места для параболического уравнения (9) с переменными коэффициентами, даже если выполнено условие $c(x, t) \leqslant 0$.

В работе А. М. Ильина [24] установлена следуюшая теорема: если $u(x, t)$ является решением уравнения (9), удовлетворяюшим условиям

1) $u(x, 1)=u_{0}(x) \rightarrow 0$ при $|x| \rightarrow \infty$;

2) уравнение (9) является равномерно параболичным (т.е. вьполнено неравенство (2));

3) коэффициенты $b_{i}(x, t)$ ограничены в полосе $H_{[1, T]}$ при любом $T>1,\left|b_{i}(x, t)\right| \leqslant$ $M$ при $|x| \leqslant r_{0}, r_{0}>0$

4) $\sum_{i=1}^{N}\left[a_{i i}(x, t)+x_{i} b_{i}(x, t)\right]>\delta>0$ для любого $t>1$ и $|x| \geqslant r_{0}>0$;

5) $c(x, t) \leqslant 0,(x, t) \in \mathbb{R}_{+}^{N+1}$, то $\lim _{t \rightarrow \infty} u(x, t)=0$ равномерно по $x$ в $\mathbb{R}^{N}$.

В [24] на примерах показано, что при невьполнении хотя бы одного из перечисленных условий 1)-5) теоремы утверждение может оказаться неверньм.

При доказательстве этой теоремы в [24] был установлен обобщенный принцип максимума, в условиях которого требуется, чтобы ограниченная функция $u(x, t)$ удовлетворяла неравенству (25) всюду в области $G=\Omega \times(0, T)$, кроме конечного числа поверхностей $S_{k}(k=1, \ldots, m)$, при этом функция $u(x, t)$ должна быть непрерьвной в замкну той области $\bar{G}$ и иметь нормальные производные на поверхностях $S_{k}$ по обе стороны этих поверхностей так, чтобы выполнялось следуюшее условие на скачок нормальной производной:

$$
\frac{\partial u}{\partial \nu_{+}}<\frac{\partial u}{\partial \nu_{-}} .
$$

При этом сохраняется классическое доказательство принципа максимума (см. [5; $\S 1$, теоремы $1,8,9])$. Отметим только, что функция $u(x, t)$ в силу $(29)$ не может принимать на поверхностях $S_{k}$ минимальное значение.

Подобным образом можно ослабить и условия теоремы 4 из [5; $\S 12]$ и взять в качестве $V(x)$ непрерьвную функцию, удовлетворяюшую неравенству $(25)$ всюду, кроме конечного числа поверхностей $S_{k}(k=1, \ldots, m)$, на которых вьполняются неравенства (29).

Используя методику работы [21], Ю. Н. Черемных установил [47] в одномерном случае точные по порядку оценки при $t \rightarrow \infty$ решения первой краевой задачи для уравнения (19) в криволинейном цилиндре с нулевыми граничными условиями. В эти оценки входит интеграл от величины, обратной квадрату переменной по $t$ ширины $\psi(t)$ соответствуюшей полуполосы.

Сформулируем один из результатов работы [47]. 
Рассмотрим уравнение (19), заданное в области $G$, граница которой $(\bar{G} \backslash G)$ состоит из отрезка $a \leqslant x \leqslant b$ оси $x$, прямой $x=0$ и гладкой кривой $x=\psi(t), t \geqslant 0$, для которой:

$$
\text { 1) } \left.\left.\psi(t) \in C^{(1)} \quad(t \geqslant 0) ; \quad 2\right) \quad 0<\psi(t) \leqslant 1 ; \quad 3\right) \quad-\frac{B}{4 H} \leqslant \psi^{\prime} \leqslant 0,
$$

и пусть $u(x, t)$ - решение уравнения $(19)$ в $G$ и вьполнены условия

$$
u(0, t)=\left.u(x, t)\right|_{x=\psi(t)}=0 \text { при } t \geqslant 0 .
$$

Тогда в области $G$ имеет место оценка

$$
|u(x, t)| \leqslant 2 \max _{0 \leqslant x \leqslant \psi(0)}|u(x, 0)| \exp \left\{-\frac{a_{0}}{2 H} \int_{0}^{t} \frac{\mathrm{d} \tau}{\psi^{2}(\tau)}\right\} .
$$

В работе [47] установлен целый ряд точных результатов об асимптотике при $t \rightarrow \infty$ решения первой краевой задачи в криволинейных по $t$ областях, а также приведены примеры, показьвающие точность полученных в [47] результатов.

Большой интерес представляет случай, когда коэффициенты $b_{i}(x, t)$ уравнения $(9)$ (или (19)) отличны от нуля и функция $\psi(t)$ стремится к бесконечности. Первый результат в случае гладких коэффициентов и одной пространственной переменной получен в работе [47]. В этой работе показано, что возможно логарифмическое расширение $G$ области при $t \rightarrow \infty$. Окончательньй результат принадлежит А. М. Ильину [48], которьй доказал, что $\psi(t)$ должно расти не быстрее чем логарифмически (см. [48; теоремы 1,3$]$ ).

В работе Р. З. Хасьминского [31] была дана классификация дифференциальных операторов вида

$$
p(x)=\sum_{i, k=1}^{N} a_{i k}(x) \frac{\partial^{2}}{\partial x_{i} \partial x_{k}}+\sum_{i=1}^{N} b_{i}(x) \frac{\partial}{\partial x_{i}}
$$

относительно принадлежности оператора $p(x)$ одному из классов $\left(A_{1}\right),\left(A_{2}\right),\left(A_{3}\right)$ (определения $\left(A_{i}\right), i=1,2,3$, мы приводим ниже) и установлена связь между стабилизацией решения задачи Коши вида

$$
\begin{gathered}
p(x) u+c(x) u-u_{t}=0, \quad(x, t) \in \mathbb{R}_{+}^{N+1}, \\
u(x, 0)=u_{0}(x), \quad x \in \mathbb{R}^{N}
\end{gathered}
$$

и принадлежностью оператора $p(x)$ одному из классов $\left(A_{i}\right), i=1,2,3$.

Предполагается, что

$$
(a(x), \xi, \xi) \geqslant \gamma(x)|\xi|^{2},
$$

где $\gamma(x)>0$ и $\gamma(x)$ непрерьвна в $\mathbb{R}^{N}, c(x) \leqslant 0$.

Пусть $\Omega$ - некоторая ограниченная область в $\mathbb{R}^{N}$ с достаточно гладкой гранищей $\partial \Omega$. Будем говорить, что оператор $p(x)(30)$ принадлежит классу $\left(A_{1}\right)$, если для области $\Omega \subset \mathbb{R}^{N}$ сушествует не менее двух различных ограниченных решений внешней задачи Дирихле для эллиптического уравнения $p(x) u=0$. Оператор $p(x)$ принадлежит классу $\left(A_{2}\right)$, если $p(x) \notin\left(A_{1}\right)$ и уравнение $p(x) u=-1$ не имеет положительного решения в $\mathbb{R}^{N} \backslash \Omega$. Оператор $p(x)$ принадлежит классу $\left(A_{3}\right)$, если $p(x) \notin\left(A_{1}\right)$ и $p(x) \notin\left(A_{2}\right)$. 
Примером оператора из класса $\left(A_{2}\right)$ может служить оператор Лапласа в $\mathbb{R}^{2}$. Оператор Лапласа в $\mathbb{R}^{N}$ при $N \geqslant 3$ дает пример оператора класса $\left(A_{1}\right)$. Примером оператора $p(x)$ из класса $\left(A_{3}\right)$ может служить оператор

$$
p(x)=\frac{\partial^{2}}{\partial x^{2}}-\operatorname{arctg} x \frac{\partial}{\partial x} .
$$

В работе [31] установлены следуюшие утверждения.

Пусть $u(x, t)$ является ограниченным решением задачи Коши (31), (32), и пусть $u_{0}(x)$ является финитной. Тогда справедливы следуюшие утверждения:

a) если $p(x) \in\left(A_{1}\right)$, то

$$
\lim _{t \rightarrow \infty} u(x, t)=0 \text { и } \int_{0}^{\infty}|u(x, t)| \mathrm{d} t<\infty ;
$$

б) если $p(x) \in\left(A_{2}\right)$, то

$$
\lim _{t \rightarrow \infty} u(x, t)=0,
$$

но при $c(x) \equiv 0$ и $u_{0}(x) \geqslant 0$

$$
\int_{0}^{\infty} u(x, t) \mathrm{d} t=\infty
$$

Пусть начальная функция $u_{0}(x)$ ограничена (но может быть не финитна), тогда:

в) если $p(x) \in\left(A_{3}\right)$ и $c(x) \equiv 0$, то

$$
\lim _{t \rightarrow \infty} u(x, t)=\int_{\mathbb{R}^{N}} u_{0}(x) l(x) \mathrm{d} x,
$$

где $l(x)>0$ - единственное решение сопряженного уравнения $p^{*}(x) l(x)=0$, для которого

$$
\int_{\mathbb{R}^{N}} l(x) \mathrm{d} x=1
$$

г) если $p(x) \in\left(A_{2}\right)$ или $p(x) \in\left(A_{3}\right), c(x) \leqslant 0$ и $c(x) \not \equiv 0$, то

$$
\lim _{t \rightarrow \infty} u(x, t)=0 .
$$

Таким образом, в случае, когда коэффициенты уравнения (31) не зависят от $t$, сформулированные выше утверждения дают достаточно полную классификацию поведения решений при $t \rightarrow \infty$, по крайней мере для случая финитных функций $u_{0}(x)$. Но если коэффициенты уравнения (31) зависят еще и от $t$, то картина зависимости поведения решения от коэффищиентов уравнения оказьвается более сложной. Это видно из цитируемых ниже результатов работы [32].

В работе [32] А. М. Ильина и Р. З. Хасьминского получены достаточные условия на коэффициенты уравнения (9), при выполнении которых остаются верными утверждения, аналогичные приведенным выше результатам а)-г) работы [31]. 
Рассмотрим дифференциальньй оператор

$$
p(x, t)=\sum_{i, k=1}^{N} a_{i k}(x, t) \frac{\partial^{2}}{\partial x_{i} \partial x_{k}}+\sum_{i=1}^{N} b_{i}(x, t) \frac{\partial}{\partial x_{i}} .
$$

Предположим, что выполнено условие

$$
(a(x, t) \xi, \xi) \geqslant \gamma(x)|\xi|^{2},
$$

где $\gamma(x)>0$ - непрерьвная функция в $\mathbb{R}^{N}, c(x, t) \leqslant 0$,

$$
\left|a_{i k}(x, t)\right| \leqslant M\left(1+|x|^{2}\right), \quad\left|b_{i}(x, t)\right| \leqslant M(1+|x|) \text { для любого } t \geqslant 0 .
$$

В теореме 1 работы [32] установлено, что если существует функция $V=V(|x|)>0$ такая, что

$$
\begin{gathered}
p(x, t) V \leqslant 0 \text { при }|x| \geqslant R>0 \text { и каждом } t>0, \\
\lim _{|x| \rightarrow \infty} V(|x|)=0
\end{gathered}
$$

то сушествует предел $\lim _{t \rightarrow \infty} u(x, t)=0$ решения задачи Коши (9), (10) равномерно по $x$ на любом компакте $K$ в $\mathbb{R}^{N}$.

ЗАмечАниЕ. Достаточным условием существования функции $V(|x|)$, удовлетворяющей (35) и (36), является сходимость следуюшего интеграла:

$$
\int_{r_{0}}^{\infty} r \exp \left\{-\int_{r_{0}}^{r} g(y) \mathrm{d} y\right\} \mathrm{d} r<\infty
$$

где

$$
g(y)=\inf _{\substack{|x|=y \\ t \geqslant 0}} \frac{\sum_{i=1}^{N}\left(a_{i i}(x, t)+b_{i}(x, t) x_{i}\right)}{\sum_{i, k=1}^{N} a_{i k}(x, t)\left(x_{i} x_{k} / y^{2}\right)} .
$$

При этом, как легко видеть, функщия

$$
V(|x|)=\int_{|x|}^{\infty} r \exp \left\{-\int_{r_{0}}^{r} g(y) \mathrm{d} y\right\} \mathrm{d} r
$$

удовлетворяет условиям (35), (36).

Предполагая, что для некоторых ограниченных областей $\Omega$ и $\Omega_{1}$ из $\mathbb{R}^{N}$ таких, что $\Omega \subset \Omega_{1}$, справедливы соотношения

$$
\begin{array}{lll}
c(x, t)<-C_{0}<0 & \text { при } & x \in \Omega, \\
c(x, t) \equiv 0 & \text { при } & x \in \Omega_{1} \backslash \Omega,
\end{array}
$$

авторы [32] устанавливают теорему 2, в которой утверждается, что решение задачи Коши (9), (10) имеет предел

$$
\lim _{t \rightarrow \infty} u(x, t)>0
$$

если существует функция $V(|x|)$ такая, что выполнены условия (35) и (36) и $\inf u_{0}(x)>0$.

Если же $u(x, t)$ - решение задачи $(31),(32)$ и $\lim _{|x| \rightarrow \infty} u_{0}(x)=k$, то

$$
\lim _{t \rightarrow \infty} u(x, t)=V(x),
$$

где $V(x)$ - единственное решение эллиптического уравнения

$$
p(x) V(x)+c(x) V(x)=0,
$$

для которого сушествует предел $\lim _{|x| \rightarrow \infty} V(x)=k$.

Основньм результатом работы [32] являются следуюшие теоремы 3 и $3^{\prime}$. 
ТеОрема 3 [32]. Если и $(x, t)$ - решение уравнения $(9)$ при $c(x, t) \equiv 0$ и $и(x, 0)=$ $u_{0}(x)$, где $u_{0}(x)$ - ограниченная функция, и при $|x| \geqslant R_{0} u t \geqslant 0$ существует функция $V(|x|, t)$, для которой:

1) $\left(p\left(x, t-t_{0}\right)-\frac{\partial}{\partial t}\right) V(|x|, t) \leqslant 0$

2) $V(|x|, 0) \geqslant 1$;

3) $\int_{0}^{\infty} V(|x|, t) \mathrm{d} t<\infty$

mо $\lim _{t \rightarrow \infty} u(x, t)=\mu$, где $\mu-$ постоянная, причем $\mu>0$, если $u_{0}(x) \geqslant 0, u$ сходимость равномерна на каждом компакте $K$ в $\mathbb{R}^{N}$.

В [32] указаны достаточные условия (в терминах коэффициентов уравнения (9)), при выполнении которых легко построить функцию $V(x, t)$, удовлетворяющую требованиям 1)-3) теоремы 3.

ТеОРема $3^{\prime}[32]$. Если $и(x, t)$ - решение уравнения $(9)$ при $c(x, t) \equiv 0$, коэффичиенты $a_{i k}(x, t)$ уравнения (9) ограничены всюду при $t \geqslant t_{0} u$

$$
\sum_{i=1}^{N}\left[a_{i i}(x, t)+b_{i}(x, t) x_{i}\right]<-\beta<0,
$$

то существует предел

$$
\lim _{t \rightarrow \infty} u(x, t)=\mu
$$

решения задачи Коши (9), (10), равномерный по $x$ накаждом компакте $K$ в $\mathbb{R}^{N}$, причем $\mu>0$, если $u_{0}(x) \geqslant 0$ в $\mathbb{R}^{N}$.

В работе [32] доказана также теорема, устанавливающая связь результатов о стабилизации решения задачи Коши (9), (10) с предельньм поведением при $y \rightarrow+\infty$ решения эллиптического уравнения в полупространстве $\mathbb{R}_{+}^{N+1}=\left\{x \in \mathbb{R}^{N}: y>0\right\}$ :

$$
a_{1}(y) V_{y y}+b(y) V_{y}+p(x) V+c(x) V=0, \quad(x, y) \in \mathbb{R}_{+}^{N+1},
$$

удовлетворяющего граничному условию

$$
V(x, 0)=V_{0}(x)
$$

где $V_{0}(x)$ - непрерывная и ограниченная в $\mathbb{R}^{N}$ функция, $p(x)$ определено в $(30)$. Здесь считается, что $a_{1}(y)>0$, a $b(y)$ удовлетворяет условию Гёльдера при всех $y>0$.

ТеОрема 7 [32]. Пусть для решения задачи Коши

$$
\begin{gathered}
p(x) u+c(x) u-u_{t}=0, \quad(x, t) \in \mathbb{R}^{N}, \\
\left.u\right|_{t=0}=u_{0}(x), \quad x \in \mathbb{R}^{N},
\end{gathered}
$$

существует предел $\lim _{t \rightarrow \infty} u(x, t)=\mu$ для каждого $x \in \mathbb{R}^{N}$, где $p(x)$ определено в (30). Если выполнены условия

$$
\begin{gathered}
\int_{0}^{\infty} \exp \left\{-\int_{0}^{t} \frac{b(y)}{a_{1}(y)} \mathrm{d} y\right\} \mathrm{d} t=\infty \\
\int_{0}^{\infty} \mathrm{d} t \int_{t}^{\infty} \frac{1}{a_{1}(u)} \exp \left\{\int_{t}^{u} \frac{b(s)}{a_{1}(s)} \mathrm{d} s\right\} \mathrm{d} u=\infty,
\end{gathered}
$$


то для решения задачи (38), (39) существует предел

$$
\lim _{y \rightarrow \infty} V(x, y)=\mu \text {. }
$$

В работе [49] изучалось поведение при большом времени $t$ положительных решений линейного параболического неравенства

$$
p(x, t) u+c(x, t) u-u_{t} \leqslant 0 \text { в } \mathbb{R}_{+}^{N+1},
$$

где оператор $p(x, t)$ определен в (33), при условии, что коэффищиент $c(x, t)$ положителен для некоторых значений $(x, t)$ в $\mathbb{R}_{+}^{N+1}$, при этом в [49] устанавливались теоремы о достаточных условиях на поведение коэффищиентов в неравенстве (40), при выполнении которых решение $u(x, t)$ этого неравенства не стабилизируется в том смысле, что справедливо

$$
\lim _{t \rightarrow \infty} u(x, t)=+\infty .
$$

В [49] установлен результат (теорема 1), согласно которому если выполнены следуюшие условия:

1) существует положительная и непрерьвная в $\mathbb{R}^{N}$ функция $\gamma(x)$, для которой имеют место неравенства (34),

2) $\left|a_{i k}(x, t)\right| \leqslant M(x),\left|b_{i}(x, t)\right| \leqslant M(x)$, где $M(x)$ - некоторая положительная и непрерывная в $\mathbb{R}^{N}$ функция,

3) сушествует функция $V(x)>0$, удовлетворяющая условиям

$$
p(x, t) V \leqslant 0
$$

при достаточно больших $x,|x| \geqslant R$, и всех $t>0$ и

$$
\lim _{|x| \rightarrow \infty} V(x)=+\infty
$$

4) коэффициент $c(x, t)$ удовлетворяет неравенству $c(x, t) \geqslant C_{1}$ при $|x|<\delta$, где $C_{1}>0, \delta>0$,

$5)$ функция $u(x, t)$ является решением неравенства $(40)$ и $u(x, 0) \not \equiv 0$, то справедливо предельное соотношение (41).

Более точно, сушествуют функция $\psi(x)>0$ и число $\beta>0$, для которых справедливо неравенство

$$
u(x, t) \geqslant \psi(x) \exp (\beta t) \text { при } t \geqslant 1 .
$$

ЗАмечАниЕ. Согласно терминологии, введенной Н. Мейерсом и Дж. Серрином [50], функцию $V(x)>0$, удовлетворяюшую условиям (42) и (43), назьвают антибарьером, отвечающим неравенству (40) (или уравнению (9)) на бесконечности.

Если функция $V(x)>0$ удовлетворяет условиям (35) и (36), то эту функцию $V(x)$ называют барьером, отвечающим неравенству (40) (или уравнению (9)) на бесконечности [50].

В случае оператора Лапласа барьер существует при $N \geqslant 3$, а антибарьер - при $N=1,2$.

Итак, в работе [49] (теорема 1) доказано, что если антибарьер существует и $c(x, t) \geqslant 0, c(x, t) \not \equiv 0$, то положительное решение неравенства (40) стремится к бесконечности при $t \rightarrow \infty$ экспоненциально.

Если для уравнения (9) существует барьер, то это утверждение, вообще говоря, неверно (см. [49; теорема 4]), и если дополнительно предположить, что коэффищиент $c(x, t)$ является достаточно большим, то (см. [49; теорема 2]) справедливо предельное равенство (41). 
ЗАмЕчАнИЕ. Используя понятие барьера (антибарьера) для уравнения (9), мы могли бы дать другую, эквивалентную, переформулировку теорем 4 и 5 из $\S 12$ работы [5] и теорем 1 и 2 из работы [32] в терминах существования антибарьера (или соответственно барьера) для уравнения (9). Мы этого не будем делать и отметим только, что понятие антибарьера было впервые введено в работе М. Кржижанского [51] и затем с успехом использовалось в качественной теории уравнений в частных производных другими авторами, среди которых мы особо выделим работы С. Симода и М. Нагумо [52], а также Н. Мейерса и Дж. Серрина [50] и работу П. Бесала и Р. Файфа [49] и Т. Кузано [53].

Далее мы обобшим концепцию антибарьера, перенеся это понятие с классических решений суперпараболических неравенств на обобшенные решения соответствуюших суперпараболических неравенств с дивергентной эллиптической частью. Это мы сделаем ниже, в п. $4^{\circ}$ настоящего параграффа.

$3^{\circ}$. О необходимых и достаточных условиях стабилизации решения задачи Коши для параболических уравнений с младшими членами. В работе В. В. Жикова [54] рассмотрены параболические уравнения, коэффициенты которых либо суть гладкие периодические функции аргументов $x$ и $t$, либо не зависят от $t$ и являются квазипериодическими функциями аргумента $x$.

В работе [54] изучены следуюшие задачи Коши:

$$
\begin{gathered}
L(x, t) u+(b(x, t), \nabla u)-u_{t}=0 \text { в } \mathbb{R}_{+}^{N+1}, \\
u(x, 0)=u_{0}(x) \in L^{\infty}\left(\mathbb{R}^{N}\right)
\end{gathered}
$$

и

$$
\begin{gathered}
L(x, t) u+(b(x, t), \nabla u)+c(x, t) u-u_{t}=0 \quad \text { в } \mathbb{R}_{+}^{N+1}, \\
u(x, 0)=u_{0}(x) \in L^{\infty}\left(\mathbb{R}^{N}\right) .
\end{gathered}
$$

Предполагается, что вьполнено условие (2) параболичности и что решения задач $(44),(45)$ и (46), (47) являются ограниченньми функциями в $\mathbb{R}_{+}^{N+1}$.

Наряду с задачей (44), (45), в [54] рассмотрен и ее частный случай

$$
\begin{gathered}
\mathscr{L}(x, t) u-u_{t}=0, \\
u(x, 0)=u_{0}(x) \in L^{\infty}\left(\mathbb{R}^{N}\right) .
\end{gathered}
$$

Как отмечено в [54], одной из желательных формулировок задач об асимптотике и стабилизации является следуюшая: найти параболический оператор

$$
B=\sum_{i, k=1}^{N} \widehat{a}_{i k} \frac{\partial^{2}}{\partial x_{i} \partial x_{k}}+\sum_{i=1}^{N} \lambda_{i} \frac{\partial}{\partial x_{i}}-\frac{\partial}{\partial t}
$$

с постоянными коэффищиентами $\widehat{a}_{i k}, \lambda_{i}$ и периодическую функцию $\rho(x)$ такие, что если $V(x, t)$ являетс решением задачи Коши

$$
\begin{gathered}
B V=0 \quad \text { в } \mathbb{R}_{+}^{N+1}, \\
V(x, 0)=\rho(x) u_{0}(x), \quad x \in \mathbb{R}^{N},
\end{gathered}
$$


то для любого начального значения $u_{0}(x) \in L^{\infty}\left(\mathbb{R}^{N}\right)$ сушествует предел

$$
\lim _{t \rightarrow \infty}(u(x, t)-V(x, t))=0
$$

т.е. имеет место теорема о равностабилизации решений задач Коши (44), (45) и (51), (52).

При дополнительных условиях, при которых вектор $b=\left(b_{1}, \ldots, b_{N}\right)$ мало отличается от постоянного, т.е. имеет вид $b=b^{1}+\varepsilon$, где $b^{1}-$ постоянньй вектор, $\varepsilon>0-$ достаточно малое, такие результаты о равностабилизации (53) имеют место.

Предполагается [54], что коэффииценты уравнений (44) и (46) являются достаточно гладкими и периодическими с периодом единица по каждому аргументу $\left(x_{1}, \ldots, x_{N}, t\right)$.

TеОРема 1 [54]. Пусть вектор $b=\left(b_{1}, \ldots, b_{N}\right)$ мало отличается от постоянного вектора $b^{1}$. Тогда найдутся параболический оператор вида (50) с постоянными коэффициентами и гладкая периодическая функиия $\rho(x)$ такие, что если и $(x, t)$ - решение задачи (44), (45), а V(x,t) - решение задачи (51), (52), то имеет место предельное соотношение (53).

ТеОРема 2 [54]. Пусть вектор $b=\left(b_{1}, \ldots, b_{N}\right)$ мало отличается от постоянного, а функиия $c(x, t)$ мало отличается от константы. Тогда существуют параболический оператор $B$ вида (50) с постоянными коэффициентами, вещественное число $\mu$ и гладкие периодические положительные функчии $\rho(x), q(x, t)$ такие, что если и( $x, t)$ - решение задачи (46), (47), а $V(x, t)$ - решение задачи (51), (52), то имеет место равенство

$$
\lim _{t \rightarrow \infty} \sup _{x \in \mathbb{R}^{N}}\left|e^{\mu t} u(x, t)-q(x, t) V(x, t)\right|=0 .
$$

Теоремы о равностабилизации позволяют найти критерий стабилизации, изучив “методом усреднения", принадлежашего В.В. Жикову [19], решение задачи Коши (51), (52) с постоянньми коэффициентами.

На этом пути в [54] получен следующий результат.

Теорема 3 [54]. Для решения задачи Коши (44), (45) предел

$$
\lim _{t \rightarrow+\infty} u(x, t)=l \quad \text { при каждом } \quad x \in \mathbb{R}^{N}
$$

существует тогда и только тогда, когда существует предел

$$
\lim _{R \rightarrow \infty} \frac{\int_{\left(B_{1} y, y\right) \leqslant R^{2}} \rho(y) u_{0}(x+y) \mathrm{d} y}{\int_{\left(B_{1} y, y\right) \leqslant R^{2}} \rho(y) \mathrm{d} y},
$$

где $B_{1}$ - матрица, обратная матрице $\widehat{a}_{i k}$ оператора $(50),(x, y)-$ скалярное произведение в $\mathbb{R}^{N}$.

Аналогичная теорема справедлива и для решения задачи (46), (47), при этом предел (53) заменяется на предел

$$
\lim _{t \rightarrow \infty} e^{\mu t} u(x, t) q^{-1}(x, t)=l
$$


В работе [54] получены результаты и для квазипериодических коэффициентов. В этом случае уравнения считаются стационарными, т.е. коэффициенты не зависят от $t$.

Переходя к формулировке этих результатов, напомним, что функция $f(x)=$ $f\left(x_{1}, \ldots, x_{N}\right)$ назьвается квазипериодической, если она допускает представление

$$
f(x)=\varphi\left(\omega^{1} \cdot x, \ldots, \omega^{m} \cdot x\right)
$$

где $\varphi\left(y_{1}, \ldots, y_{m}\right)$ - непрерывная периодическая (с периодом единица по каждому аргументу $\left.y_{1}, \ldots, y_{m}\right)$ функция, $\omega^{1}, \ldots, \omega^{m}$ - элементы из $\mathbb{R}^{N}, \omega^{i} \cdot x=\omega_{1}^{i} x_{1}+\cdots+$ $\omega_{N}^{i} x_{N}$. Частоты $\omega^{1}, \ldots, \omega^{m}$ можно считать рационально независимыми в том смысле, что $k_{i} \omega_{j}^{i} \neq 0$ для любого $j=1, \ldots, N$ и любого целочисленного вектора $k=$ $\left(k_{1}, \ldots, k_{m}\right) \neq 0$. Следствием независимости является равенство

$$
\langle f\rangle=\langle\varphi\rangle
$$

где $\langle f\rangle$ - среднее значение почти периодической функции $f,\langle\varphi\rangle$ - среднее значение периодической функции $\varphi$.

В [54] установлен следуюший результат.

Теорема 4 [54]. Пусть $и(x, t)$ - решение задачи Коши

$$
\sum_{i, k=1}^{N} a_{i k}(x) u_{x_{i} x_{k}}-u_{t}=0, \quad u(x, 0)=u_{0}(x) \in L^{\infty}\left(\mathbb{R}^{N}\right)
$$

а матрица $\left\{a_{i k}(x)\right\}$ квазипериодична и мало отличается от постоянной. Тогда найдутся квазипериодическая функиия $\rho(x)$ и положительно определенная постоянная матрица $\left\{\widehat{a}_{i k}\right\}$ такие, что если $V(x, t)$ есть решение задачи Коши

$$
\sum_{i, k=1}^{N} \widehat{a}_{i k} V_{x_{i} x_{k}}-V_{t}=0, \quad V(x, 0)=\rho(x) u_{0}(x)
$$

то имеет место равенство (53).

В задачах, связанных с квазипериодическими функциями, обычно накладывается дополнительное условие на частоты $\omega^{i}$, а именно требование

$$
\left|k_{i} \omega_{j}^{i}\right| \geqslant C^{-1}|k|^{-\tau} \quad(j=1, \ldots, m)
$$

где $C$ и $\tau$ - положительные постоянные, $k=\left(k_{1}, \ldots, k_{m}\right)$ - любой ненулевой целочисленный вектор.

В теореме 4 такого рода условия не накладываются, однако рассмотрение уравнений с младшими членами неизбежно приводит к условиям (59). 
ТЕОрема 5 [54]. Пусть коэффициенты $a_{i k}, b_{i}$, с являются квазипериодическими и выполнено условие (59), $b_{i}$ и с мало отличаются от констант. Тогда найдутся параболический оператор с постоянными коэффичиентами вида (50), вещественное число $\mu$ и квазипериодическая функиия $\rho(x)$ такие, что если и $(x, t)-$ решение задачи Коши (46), (47), V(x,t) - решение задачи Коши (51), (52), то имеет место соотношение (54). При этом если с $\equiv 0$, то $\mu=0$.

В работе К.В. Валикова [55] теоремы, доказанные В. В. Жиковым в [54], распространены на некоторые уравнения параболического типа с младшими коэффициентами при меньших ограничениях.

Сформулируем основные результаты работы [55].

В полупространстве $\mathbb{R}_{+}^{N+1}$ рассматривается уравнение

$$
\sum_{i, k=1}^{N} a_{i k}(t) u_{x_{i} x_{k}}+\sum_{i=1}^{N} b_{i}(x, t) u_{x_{i}}-u_{t}=0
$$

с измеримьми ограниченными коэффициентами $a_{i k}(t)=a_{k i}(t), b_{i}(x, t)$ и начальное условие

$$
u(x, 0)=u_{0}(x),
$$

где $u_{0}(x)$ - измеримая функция, $u_{0}(x) \in L^{\infty}\left(\mathbb{R}^{N}\right)$.

Предполагается выполненным условие (2) равномерной параболичности.

Пусть $Q=\Omega \times(0, T), \Omega$ - область в $\mathbb{R}^{N}$. Вводится банахово пространство $L_{q, r}(Q)$ $(q, r \in[2,+\infty))$ измеримых в $Q$ функций $u(x, t)$ с нормой

$$
\|u\|_{q, r}=\left[\int_{0}^{T}\left(\int_{\Omega}|u(x, t)|^{q} \mathrm{~d} x\right)^{r / q} \mathrm{~d} t\right]^{1 / r} .
$$

Предполагается [55], что коэффищиенты $b_{i}(x, t)$ имеют вид

$$
b_{i}(x, t)=b_{i, 1}(x, t)+b_{i, 2}(x, t),
$$

где

$$
\begin{gathered}
b_{i, 1}(x, t) \in L_{q, r}\left(\mathbb{R}_{+}^{N+1}\right), \quad \frac{N}{2 q}+\frac{1}{r}=\frac{1}{2}, \\
\left|b_{i, 2}(x, t)\right| \leqslant b(x) \in L_{N}\left(\mathbb{R}^{N}\right) \text { при } N \geqslant 3, \\
b_{0,2}(x, t)=0 \text { при } \quad N=1,2 .
\end{gathered}
$$

В формулируемых ниже теоремах 1 и 2 из [55] предполагается, что число

$$
\mu_{0}=\|b\|_{N, \mathbb{R}^{N}}
$$

удовлетворяет условию малости

$$
\mu_{0}<\frac{N-2}{N^{2}(2 N-2) \lambda} \text { при } N \geqslant 3,
$$

где $\lambda$ - постоянная из неравенства (2). 
ТеОрема 1 [55]. Пусть постоянные $\widehat{a}_{i k}$ такие, что

$$
\lim _{R \rightarrow \infty} \frac{1}{R} \int_{0}^{R} a_{i k}(t) \mathrm{d} t=\widehat{a}_{i k}
$$

Тогда для существования поточечного в $\mathbb{R}^{N}$ предела

$$
\lim _{t \rightarrow \infty} u(x, t)=l
$$

решения задачи (60), (61) необходимо и достаточно, чтобы существовал предел

$$
\lim _{R \rightarrow \infty} \frac{\sqrt{\operatorname{det} B}}{\omega_{N} R^{N}} \int_{(B x, x)<R^{2}} u_{0}(y) \mathrm{d} x=l,
$$

где $B$ - матрица, обратная матриче $\widehat{a}_{i k}(65),(x, y)$ - скалярное произведение $\boldsymbol{\beta} \mathbb{R}^{N}$.

В теореме 2 из [55] на $a_{i k}(t)$ не налагается никаких ограничений, кроме измеримости и равномерной параболичности, однако от коэффициентов $b_{i}(x, t)$ дополнительно требуется, чтобы $b_{i, 2}(x, t) \equiv 0$ для всех размерностей.

Tеорема 2 [55]. Для равномерной стабилизации и $(x, t)$ задачи $(60),(61)$ к постоянной $l$ (66) необходимо и достаточно, чтобь равномерно по $\beta \in \mathbb{R}^{N}$ существовал предел

$$
\lim _{R \rightarrow \infty} \frac{1}{R^{N}} \int_{\beta+K_{R}} u_{0}(x) \mathrm{d} x=l,
$$

где $K_{R}-$ куб в $\mathbb{R}^{N}, K_{R}=\left\{x: x \in \mathbb{R}^{N}, 0<x_{i}<R, i=1, \ldots, N\right\}, \beta+K_{R}-$ сдвиг на вектор $\beta$ куба $K_{R}$.

Ограничения (63), (64) в размерностях один и два, а в теореме 2 и во всех размерностях оставляют в стороне уравнения с независимыми от $t$ коэффициентами $b_{i}$. При $N \geqslant 2$ вместо (60) в [55] рассмотрено уравнение

$$
\Delta u+\sum_{i=1}^{N} b_{i}(x) u_{x_{i}}-u_{t}=0
$$

в котором вектор $b(x)=\left(b_{1}(x), \ldots, b_{N}(x)\right)$ потенциален, т.е. имеет вид

$$
b(x)=\operatorname{grad} B(x),
$$

где $B(x)$ - ограниченная в $\mathbb{R}^{N}$ функция и $B(x) \in W_{2}^{1, \operatorname{loc}}\left(\mathbb{R}^{N}\right)$. Положим $c(x)=$ $\exp B(x)$.

ТЕОРЕМа 3 [55]. Пусть функиия $B(x)$ имеет на бесконечности радиальный предел

$$
\lim _{\omega \rightarrow \infty} B(\omega x)=\bar{B}(\varphi)
$$

для почти всех х на единичной сфере в $\mathbb{R}^{N}, \varphi-$ совокупность угловых координат в $\mathbb{R}^{N}$. Для существования точечного в $\mathbb{R}^{N}$ предела (66) задачи Коши (67), (61) необходимо и достаточно, чтобь существовал предел

$$
\lim _{R \rightarrow \infty} \frac{R^{-N} \int_{|x|<R} c(x) u_{0}(x) \mathrm{d} x}{R^{-N} \int_{|x|<R} c(x) \mathrm{d} x}=l .
$$


Теорема 4 [55]. Для существования равномерного в $\mathbb{R}^{N}$ предела (66) решения задачи Коши (67), (61) необходимо и достаточно, чтобы равномерно по $\beta \in \mathbb{R}^{N}$ существовал предел

$$
\lim _{R \rightarrow \infty} \frac{\int_{\beta+K_{R}} c(x) u_{0}(x) \mathrm{d} x}{\int_{\beta+K_{R}} c(x) \mathrm{d} x}=l .
$$

Рассмотрим одномерное уравнение

$$
\left(a(x) u_{x}\right)_{x}+b(x) u_{x}-c(x) u_{t}=0
$$

в предположении равномерной параболичности

$$
c(x) \geqslant \frac{1}{\lambda}, \quad \lambda^{-1} \leqslant a(x) \leqslant \lambda, \quad x \in \mathbb{R}^{1}, \quad \lambda>0
$$

и при условии $b(x) \in L_{2, \text { loc }}\left(\mathbb{R}^{1}\right)$.

Положим

$$
\begin{gathered}
h(x)=\exp \int_{0}^{x} \frac{b(\xi)}{a(\xi)} \mathrm{d} \xi, \quad p(x)=c(x) h(x), \\
q(x)=a(x) h(x), \quad r(x)=\int_{0}^{x} \frac{\mathrm{d} \xi}{q(\xi)}
\end{gathered}
$$

и обозначим через $s(x)$ функцию, обратную к $r(x)$. Допустим, что функция $h(x)$ ограничена в $\mathbb{R}^{1}$.

ТЕОРЕМА 5 [55]. Пусть выполнено условие

$$
\lim _{R \rightarrow \pm \infty} \frac{1}{r(R)} \int_{0}^{R} p(x) \mathrm{d} x=A_{ \pm}^{2}
$$

где $A_{ \pm}-$некоторые константы. Для поточечной стабилизации и $(x, t)$, т.е. существования предела (66) решения задачи Коши (68), (61), необходимо и достаточно, чтобы существовал предел

$$
\lim _{R \rightarrow \infty} \frac{1}{\left(A_{+}+A_{-}\right) R} \int_{s\left(-A_{-}^{-1} R\right)}^{s\left(A_{+}^{-1} R\right)} p(x) u_{0}(x) \mathrm{d} x=l .
$$

Отметим, что для уравнения (68) при $b(x) \equiv 0$ теорема о стабилизации получена в работе $\Phi$. О. Порпера, С.Д. Эйдельмана [56]. Случай $a(x) \equiv 1, b(x)=0$ в условиях сушествования пределов

$$
\lim _{R \rightarrow \pm \infty} R^{-1} \int_{0}^{R} p(x) \mathrm{d} x=p_{ \pm}^{2}, \quad \lim _{R \rightarrow \pm \infty} R^{-1} \int_{0}^{R} \frac{\mathrm{d} x}{q(x)}=q_{ \pm}^{-2}
$$

изучался в работе В.В. Жикова [19] и, с других позищий, в работе А.К. Гушина и В. П. Михайлова [57], где впервые получены интересные критерии стабилизащии в одномерном случае.

Условия (69) слабее (70), так как они могут выполняться, когда пределы (70) не сушествуют. К.В. Валиков обобшил результаты [55] в работе [58]. 
$\mathrm{B} \mathbb{R}_{+}^{N+1}$ рассматриваются две задачи Коши:

$$
\begin{gathered}
L(x, t) u+b(x, t) u-c(x, t) u_{t}=0, \quad(x, t) \in \mathbb{R}_{+}^{N+1}, \\
u(x, 0)=u_{0}(x) \in L^{\infty}\left(\mathbb{R}^{N}\right)
\end{gathered}
$$

и

$$
\begin{gathered}
L(x, t) V-c(x, t) V_{t}=0, \quad(x, t) \in \mathbb{R}_{+}^{N+1} \\
V(x, 0)=u_{0}(x) \in L^{\infty}\left(\mathbb{R}^{N}\right)
\end{gathered}
$$

Считается [58], что

$$
b_{i k}(x, t) \in L_{q, r}\left(\mathbb{R}_{+}^{N+1}\right)
$$

где норма $L_{q, r}\left(\mathbb{R}_{+}^{N+1}\right)$ введена равенством $(62)$ при $\Omega=\mathbb{R}_{+}^{N+1}$ и некоторых $q, r \geqslant 2$, связанных соотношениями (63) и

$$
\lambda^{-1} \leqslant c(x, t) \leqslant \lambda, \quad \frac{\partial c}{\partial t}(x, t) \leqslant 0, \quad \frac{\partial c}{\partial t}(x, t) \in L_{1, \mathrm{loc}}\left(\overline{\mathbb{R}}_{+}^{N+1}\right) .
$$

Будем говорить, что решения $u$ и $V$ задач $(71),(72)$ и (73), (74) близки (равномерно близки) при $t \rightarrow+\infty$, если для любого $x \in \mathbb{R}^{N}$ (равномерно по $x \in \mathbb{R}^{N}$ )

$$
\lim _{t \rightarrow \infty}(u(x, t)-V(x, t))=0
$$

Близость решений параболических уравнений исследовалась в работах [58]-[60], [72], [73]. Интересные результаты о близости получены в [59] методом В. В. Жикова [19], [54]. Этим же методом в [58] устанавливаются следуюшие результаты.

Tеорема 1 [58]. Пусть $q \in(N, \infty)$ при $N \geqslant 2 u q \in[2,+\infty)$ nрu $N=1$. Тогда решения задач (71), (72) и (73), (74) равномерно близки при $t \rightarrow+\infty$, т.е. существует предел (76) равномерно по $x \in \mathbb{R}^{N}$.

Рассмотрим случай $q=N \geqslant 3(r=\infty)$ при условии

$$
\left|b_{i}(x, t)\right| \leqslant b_{i}(x) \in L_{N}\left(\mathbb{R}^{N}\right) .
$$

ТеОрема 2 [58]. Если выполнено условие малости

$$
\|b\|_{N, \mathbb{R}^{N}}<\frac{N-2}{(2 N-2) \lambda}
$$

$\lambda$ - постоянная из (2), то решения $u(x, t), V(x, t)$ задач $(71),(72)$ u (73), (74) соответственно близки при $t \rightarrow \infty$ (т.е. существует предел $(76))$. Здесь $b(x)=$ $\left(b_{1}(x), \ldots, b_{N}(x)\right), a\|\cdot\|_{p, \Omega}-$ норма в $L_{p}(\Omega)$.

Из теорем 1 и 2 следует, что если критерий стабилизащии известен для решений задачи $(73),(74)$, то без всяких изменений этот критерий переносится на случай задачи (71), (72). Отметим, что в теореме 1 [58] отсутствует условие малости коэффициентов $b_{i}(x, t)$, которое было сформулировано в теореме 1 работы [54]. 
Однако сказанное здесь о снятии условий малости в [58] не относится к теоремам 4 и 5 работы [54], в которых рассматриваются уравнения со стационарными квазипериодическими коэффициентами.

$4^{\circ}$. О стабилизации решения задачи Коши для параболического уравнения с младшими коэффициентами и с дивергентной главной частью. $\mathrm{B} \mathbb{R}_{+}^{N+1}$ рассмотрим задачу Коши для уравнения

$$
\sum_{i, k=1}^{N}\left(a_{i k}(x) u_{x_{i}}\right)_{x_{k}}+\sum_{i=1}^{N} b_{i}(x) u_{x_{i}}+c(x) u-u_{t}=0
$$

или, кратко,

$$
L(x) u+(b(x), \nabla u)+c(x) u-u_{t}=0, \quad(x, t) \in \mathbb{R}^{N+1},
$$

с начальньм условием

$$
u(x, 0)=u_{0}(x), \quad x \in \mathbb{R}^{N},
$$

где $u_{0}(x)$ - непрерьвная и ограниченная в $\mathbb{R}^{N}$ функция, $\left|u_{0}(x)\right| \leqslant C$.

Будем считать, что коэффициенты уравнения (77) измеримы и ограничены в $\mathbb{R}^{N}$, выполнено условие (2) и

$$
c(x) \leqslant 0 \quad \text { в } \quad \mathbb{R}^{N} .
$$

Далее мы сформулируем точные достаточные условия на младшие коэффициенты уравнения (77), которые гарантируют стабилизацию к нулю

$$
\lim _{t \rightarrow \infty} u(x, t)=0
$$

равномерно по $x$ на любом компакте $K \subset \mathbb{R}^{N}$ решения задачи Коши $(77),(78)$ при любой ограниченной начальной функции $u_{0}(x)$ (теоремы $\left.1-3\right)$, и точные достаточные условия на младшие коэффициенты уравнения (77), которые гарантируют сушествование предела (80) решения задачи $(77),(78)$ и для любой непрерьвной функции, удовлетворяющей условию роста

$$
\left|u_{0}(x)\right|<C\left(1+|x|^{m}\right), \quad C>0, \quad m>0
$$

(теоремы 4 и 5 ).

ЗАмечАниЕ. Для случая, когда в уравнении (77) $L=\Delta$ - оператор Лапласа, результаты, аналогичные формулируемьм ниже теоремам 1 и 2 , установлены в [33]. Теорема 3 является новой, и мы приводим ее доказательство в $\oint 4$. Для случая, когда в уравнении (77) коэффициенты $b_{i}(x) \equiv 0$, утверждения, аналогичные формулируемым ниже теоремам 4 и 5 , доказаны в [34].

Будем говорить, что коэффициенты $b_{k}(x)(k=1, \ldots, N)$ удовлетворяют условиям $\left(\mathrm{B}_{1}\right)$, если сушествуют $B>0$ и $\varepsilon \in(0,1)$, для которых

$$
\sup _{x \in \mathbb{R}^{N}}(1+|x|)^{1+\varepsilon}\left|b_{k}(x)\right|=B \text {. }
$$


Будем говорить, что коэффициенты $b_{k}(x)(k=1, \ldots, N)$ удовлетворяют условиям $\left(\mathrm{B}_{2}\right)$, если сушествует постоянная $B>0$, для которой

$$
\sup _{x \in \mathbb{R}^{N}}(1+|x|) \sum_{i=1}^{N}\left|b_{i}(x)\right|=B .
$$

Будем говорить, что ограниченньй в $\mathbb{R}^{N}$ коэффициент $c(x)$ удовлетворяет условию $\left(\mathrm{C}_{1}\right)$, если сушествует постоянна $\alpha>0$, для которой

$$
c(x) \leqslant-\alpha^{2} \text { при }|x|<1 .
$$

Будем говорить, что ограниченньй в $\mathbb{R}^{N}$ коэффищиент $c(x)$ удовлетворяет условию $\left(\mathrm{C}_{2}\right)$, если сушествует постоянная $\alpha>0$, для которой

$$
c(x) \leqslant-\frac{\alpha^{2}}{|x|^{2}} \text { при }|x|>1 .
$$

Будем говорить, что ограниченный в $\mathbb{R}^{N}$ коэффищиент $c(x)$ удовлетворяет условию $\left(\mathrm{C}_{3}\right)$, если сушествуют постоянные $\alpha>0$ и $S>0$, для которых

$$
c(x) \leqslant-\frac{\alpha^{2}}{|x|^{2}(\ln |x|)^{S}} \text { при }|x|>2 .
$$

Имеет место следуюший результат [33], [34].

Tеорема 1. Пусть $N=1$ или $N=2$, коэффициенты $b_{i}(x)(i=1, \ldots, N)$ уравнения (77) удовлетворяют условиям $\left(\mathrm{B}_{1}\right)$, а коэффициент $c(x)$ удовлетворяет условию $\left(\mathrm{C}_{1}\right)$. Тогда решение задачи Коши $(77),(78)$ стабилизируется $к$ нулю (т.е. существует предел (80)) равномерно по $x$ на каждом компакте $K$ в $\mathbb{R}^{N}$.

ЗАмечАниЕ 1 . Если в уравнении $(77) c(x) \equiv 0$, то утверждение теоремы 1 может не выполняться.

Действительно, решение задачи Коши $\Delta u-u_{t}=0,\left.u\right|_{t=0}=1$ имеет вид $u(x, t) \equiv 1$, и оно не стабилизируется к нулю ни в одной точке $x \in \mathbb{R}^{N}$.

ЗАмечАние 2 . Условие $\left(\mathrm{C}_{1}\right)$ на $c(x)$ можно сформулировать иначе: сушествует постоянная $\alpha>0$ такая, что неравенство (84) имеет место при всех $x$, для которых $\left|x-x_{0}\right|<1$. Однако с помошью линейной замены мы легко сведем этот случай $\mathrm{K}$ случаю, когда $x_{0}=0$.

ЗАмечаниЕ 3 . Теорема 1 не имеет места при размерностях $N \geqslant 3$, ибо справедливо следующее утверждение, доказанное нами в [33].

Лемма 1. При $N \geqslant 3$ существуют коэффициент $c(x)$ уравнения

$$
\Delta u+c(x) u-u_{t}=0, \quad(x, t) \in \mathbb{R}_{+}^{N+1},
$$

удовлетворяющий условию $\left(\mathrm{C}_{1}\right)$, и ограниченная в $\mathbb{R}^{N}$ начальная функиия

$$
u(x, 0)=u_{0}(x), \quad x \in \mathbb{R}^{N},
$$

для которых решение задачи Коши (87), (88) не имеет предела (80) ни в одной mочке $x \in \mathbb{R}^{N}$.

С тем чтобы получить аналог теоремы 1 при $N \geqslant 3$, мы усилим условия на коэффициенты уравнения (77). 
ТеОрема 2. Если коэффициенты $b_{i}(x)(i=1, \ldots, N)$ уравнения $(77)$ удовлетворяют условию $\left(\mathrm{B}_{2}\right)$, коэффициент $c(x)$ удовлетворяет условию $\left(\mathrm{C}_{2}\right)$, то решение задачи Коши (77), (78) стабилизируется к нулю равномерно по х на любом компакте $K$ в $\mathbb{R}^{N}$.

Следующее утверждение, доказанное нами в [33], показьвает, что утверждение теоремы 1 нельзя усилить, заменив в нем стабилизацию (80), равномерную на каждом компакте $K$ в $\mathbb{R}^{N}$, на стабилизацию, равномерную во всем $\mathbb{R}^{N}$.

Лемма 2. При $N=1$ или $N=2$ существуют коэффициент с $(x)$, удовлетворяюший условию $\left(\mathrm{C}_{1}\right)$, и ограниченная в $\mathbb{R}^{N}$ начальная функиия, для которых решение задачи Коши (87), (88) не имеет равномерного в $\mathbb{R}^{N}$ предела (80).

$\mathrm{B} \mathbb{R}_{+}^{N+1}$ рассмотрим решение уравнения

$$
\Delta u+\sum_{i=1}^{N} b_{i}(x) u_{x_{i}}+c(x) u-u_{t}=0,
$$

удовлетворяющее условию

$$
u(x, 0)=u_{0}(x), \quad x \in \mathbb{R}^{N} .
$$

Лемма 3. I) При $N=1$ существуют ограниченный в $\mathbb{R}^{1}$ коэффичиент $b_{1}(x)$, имеющий при $|x| \geqslant 1$ вид

$$
b_{1}(x)= \begin{cases}\frac{\beta}{x} & \text { при } x \geqslant 1 \text { u } \beta>1, \\ -\frac{\beta}{x} & \text { при } x \leqslant 1 \text { u } \beta<-1,\end{cases}
$$

и коэффициент $c(x)$, удовлетворяющий условию $\left(\mathrm{C}_{1}\right)$, и ограниченная в $\mathbb{R}^{1}$ начальная функиия $u_{0}(x)$, для которых решение задачи Коши (89), (90) не стабилизируется к нулю ни в одной точке $x \in \mathbb{R}^{1}$.

II) При $N=2$ существуют ограниченные в $\mathbb{R}^{N}$ коэффициенты $b_{1}(x, y), b_{2}(x, y)$, имеющие при $x^{2}+y^{2} \geqslant 1$ следующий вид:

$$
b_{1}(x, y)=\frac{x}{\sqrt{x^{2}+y^{2}}}, \quad b_{2}(x, y)=\frac{y}{\sqrt{x^{2}+y^{2}}},
$$

коэффициент $c(x, y)$, удовлетворяющий условию $\left(\mathrm{C}_{1}\right)$, и ограниченная в $\mathbb{R}^{2}$ начальная функиия $u_{0}(x, y)$, для которых решение задачи Коши (89), (90) не стабилизируется $к$ нулю ни в одной точке $x, y \in \mathbb{R}^{2}$.

Ради краткости мы докажем в $§ 4$ лемму 3 только в случае $N=2$. Лемма 3 утверждает, что условие $\left(\mathrm{B}_{1}\right)$ на коэффициенты уравнения $(77)$ в теореме 1 является точным, ибо в условии $\left(\mathrm{B}_{1}\right)$ нельзя положить $\varepsilon=0$.

Лемма 4. При $N \geqslant 3$ существуют коэффиииент $c(x)$, удовлетворяющий условию $\left(\mathrm{C}_{2}\right)$, и ограниченная в $\mathbb{R}^{N}$ начальная функция $u_{0}(x)$, для которых решение задачи Коши (87), (88) не имеет равномерного в $\mathbb{R}^{N}$ предела (80).

Таким образом, лемма 4, доказанная в [33], является аналогом леммы 2 к теореме 1 [33], и она утверждает, что в теореме 2 тоже нельзя заменить существование предела (80), равномерного на компактах $K$ в $\mathbb{R}^{N}$, на существование равномерного в $\mathbb{R}^{N}$ предела (80). 
Лемма 5. Существуют ограниченные в $\mathbb{R}^{N}$ коэффициенты $b_{i}(x)(i=1, \ldots, N)$, имеющие при $|x| \geqslant 1$ следующий вид:

$$
b_{i}(x)=m \frac{x_{i}}{|x|^{2-\varepsilon}}, \quad 0<\varepsilon<1, \quad m>0,
$$

коэффициент $c(x)$, удовлетворяющий условию $\left(\mathrm{C}_{2}\right)$, и ограниченная в $\mathbb{R}^{N}$ начальная функиия $u_{0}(x)$, для которых решение задачи Коши (89), (90) не стабилизируется $к$ нулю при $t \rightarrow \infty$ ни в одной точке $x \in \mathbb{R}^{N}$.

Лемма 5 показьвает, что условие $\left(\mathrm{B}_{2}\right)$ на коэффициенты $b_{i}(x)$ в теореме 2 является точным. Лемма 5 будет доказана в $\S 4$.

ЛЕмма 6. Если коэффициент $c(x)$ в уравнении (87) имеет вид

$$
c(x)= \begin{cases}-\alpha^{2}|x|^{-2-\varepsilon} & \text { npu }|x|>1,0<\varepsilon<1, \\ 0 & \text { npu }|x| \leqslant 1\end{cases}
$$

то существует ограниченная в $\mathbb{R}^{N}$ начальная функция $u_{0}(x)$, для которой решение задачи Коши (87), (88) не стабилизируется $\kappa$ нулю ни в одной точке $x \in \mathbb{R}^{N}$.

Лемма 6 доказана нами в [33], и она показьвает, что условия $\left(\mathrm{C}_{2}\right)$ на коэффищиент $c(x)$ в теореме 2 являются точными по порядку убывания относительно $|x|^{-2}$ при $|x| \rightarrow \infty$. Однако если наложить условия $\left(\mathrm{C}_{3}\right)$ на коэффициент $c(x)$, то можно доказать, что теорема 2 допускает уточнение (теорема 3 ) для задачи $(89),(90)$.

Имеет место следуюшее утверждение.

ТЕОрема 3. Если $N \geqslant 3$ и коэффичиенты $b_{i}(x)(i=1, \ldots, N)$ удовлетворяют условиям $\left(\mathrm{B}_{2}\right)$, а коэффициент $c(x)$ удовлетворяет условиям $\left(\mathrm{C}_{3}\right)$ при $S \in(0,1]$, то решение задачи Коши (89), (90) стабилизируется к нулю равномерно по $x$ на любом компакте $K$ в $\mathbb{R}^{N}$.

Эта теорема является новой и будет доказана в $\S 3$.

Неравенство $0<S \leqslant 1$ в теореме 3 является точным, ибо имеет место следующее утверждение.

Лемма 7. При $N \geqslant 3$ существуют коэффициенты $b_{i}(x)(i=1, \ldots, N)$, удовлетворяющие условиям $\left(\mathrm{B}_{2}\right)$, коэффичиент $c(x)$, удовлетворяющий условиям $\left(\mathrm{C}_{3}\right)$ при $S>1$, и ограниченная в $\mathbb{R}^{N}$ начальная функиия, для которьх решение задачи Коши (89), (90) не стабилизируется $\kappa$ нулю ни в одной точке $x \in \mathbb{R}^{N}$.

Лемма 7 будет доказана в $\S 4$.

Теперь перейдем к рассмотрению случая, когда начальная функция $u_{0}(x)$ в задаче Коши (77), (78) удовлетворяет условию роста (81).

Имеет место следуюшее утверждение [35].

ТЕОРема 4. Если и ${ }_{0}(x)$ удовлетворяет условию (81) и коэффичиенты $b_{i}(x)$ $(i=1, \ldots, N)$ удовлетворяют условиям $\left(\mathrm{B}_{2}\right)$, тогда существует постоянная $\alpha_{0}^{2}=\alpha_{0}^{2}(m, B, N, \lambda)>0$ такая, что если для коэффициента с $(x)$ выполнено условие $\left(\mathrm{C}_{2}\right)$ nрu

$$
\alpha^{2}>\alpha_{0}^{2},
$$


то решение задачи Коши (77), (78) имеет предел (80) равномерно по х на каждом компакте $K$ в $\mathbb{R}^{N}$.

В случае, когдав уравнении (77) $L=\Delta$, т.е. главная часть (77) - оператор Лапласа, мы указываем точный вид постоянной $\alpha_{0}^{2}$ в условии (93) теоремы 4.

Теорема 5 [35]. Если начальная функция $u_{0}(x)$ удовлетворяет условию роста (81), в уравнении $(77) L(x)=\Delta$, коэффичиенты $b_{i}(x)(i=1, \ldots, N)$ удовлетворяют условию $\left(\mathrm{B}_{2}\right)$, тогда если $c(x)$ удовлетворяет условию $\left(\mathrm{C}_{2}\right)$ при

$$
\alpha^{2}>\alpha_{0}^{2}=m(m+N-2+B)
$$

то решение задачи Коши (77), (78) имеет предел (80) равномерно по х на каждом компакте $K$ в $\mathbb{R}^{N}$.

Теоремы 4 и 5 будут доказаны в $\S 3$. В случае, когда в уравнении $(77) b_{i}(x)=0$, а для коэффищиента $c(x, t)$ вьполнено условие $\left(\mathrm{C}_{2}\right)$ при всех $t>0$, теорема 4 доказана в работе [34].

Неравенство (94) является точным, как это вытекает из следующего утверждения, которое доказьвается аналогично лемме 2 из [35].

Лемма 8. Для любых $m>0, B>0$ существуют ограниченные в $\mathbb{R}^{N}$ коэффиииенты $b_{i}(x)(i=1, \ldots, N)$, имеющие при $|x|>2$ следующий вид:

$$
b_{i}(x)=B \frac{x_{i}}{|x|^{2}},
$$

и коэффициент $c(x)$, удовлетворяющий условию $\left(\mathrm{C}_{2}\right)$ при

$$
\alpha^{2}=\alpha_{0}^{2}=m(m+N-2+B),
$$

для которых решение задачи Коши $(77),(78)$ с $L(x)=\Delta$ не стабилизируется $\kappa$ нулю ни в одной точке $x \in \mathbb{R}^{N}$.

ЛЕмма 9. Для любых $m>0, B>0$ существуют начальная функиия $u_{0}(x)$, удовлетворяющая условию (81), коэффициенты $b_{k}(x)(k=1, \ldots, N)$, удовлетворяющие условиям $\left(\mathrm{B}_{2}\right)$, и коэффициент $c(x)$, удовлетворяющий условию $\left(\mathrm{C}_{2}\right)$ при

$$
\alpha^{2}>m(m+N-2+B),
$$

для которых решение задачи Коши (89), (90) не имеет равномерного в $\mathbb{R}^{N}$ предела (80).

Лемма 9 (см. [35]) является аналогом лемм 1 и 4 и показьвает, что утверждение теорем 4 и 5 нельзя усилить и получить в условиях теорем 4 и 5 существование равномерного в $\mathbb{R}^{N}$ предела (80).

Доказательства теорем $1-5$, сформулированных в настоящем пункте, основаны на применении понятия обобщенного решения (антибарьера), отвечающего уравнению $(77)$, т.е. такого $V(x)$, что:

$$
\text { 1) } V(x)>0 ; \quad \text { 2) } \quad V(x) \in W_{2}^{1, \text { loc }}\left(\mathbb{R}^{N}\right) ; \quad \text { 3) } \lim _{t \rightarrow \infty} V(x)=\infty
$$

и $V(x)$ удовлетворяет стационарному уравнению (13) в смысле тождества (14). Эти доказательства будут приведены в $§ 3$.

При доказательстве теорем 1-5 применяются следуюшие утверждения, которые имеют и самостоятельный интерес. 
ЛЕмма 10. Пусть выполнены условия теоремы 1, тогда существует функиия $\Gamma(x)$ такая, что:
1) $\Gamma(x)>0$ в $\mathbb{R}^{N}$
2) $\quad \Gamma(x) \in W_{2}^{1, \text { loc }}\left(\mathbb{R}^{N}\right)$;
3) $\lim _{|x| \rightarrow \infty} \Gamma(x)=+\infty$;

4) $\int_{\mathbb{R}^{N}}[(a \nabla \Gamma, \nabla \varphi)-\varphi((\nabla \Gamma, b)+c \Gamma)] \mathrm{d} x=0$

для любой финитной функиии $\varphi(x)$ из $C_{0}^{\infty}\left(\mathbb{R}^{N}\right)$.

ЛЕмма 11. Пусть выполнены условия теоремь 2, тогда существует функиия $\Gamma(x)$ такая, что:
1) $\quad \Gamma(x)>0$ в $\mathbb{R}^{N}$
2) $\quad \Gamma(x) \in W_{2}^{1, \text { loc }}\left(\mathbb{R}^{N}\right)$
3) $\lim _{|x| \rightarrow \infty} \Gamma(x)=+\infty$;

4) $\int_{\mathbb{R}^{N}}[(a \nabla \Gamma, \nabla \varphi)-\varphi((\nabla \Gamma, b)+c \Gamma)] \mathrm{d} x=0$

для любой финитной функиии $\varphi(x)$ из $C_{0}^{\infty}\left(\mathbb{R}^{N}\right)$.

ЛЕмма 12. Пусть выполнены условия теоремы 4, тогда для каждого $m>0$ существует постоянная $\alpha_{0}=\alpha_{0}(m, N, B, \lambda)>0$ и существует отвечающее этому $\alpha_{0}$ решение уравнения

$$
L(x) \Gamma+(b(x), \nabla \Gamma)+q_{\alpha_{0}}(x) \Gamma=0 \quad \text { e } \quad \mathbb{R}^{N},
$$

$2 \partial e$

$$
q_{\alpha_{0}}(x)= \begin{cases}-\frac{\alpha_{0}^{2}}{|x|^{2}} & \text { npu }|x|>2, \\ 0 & \text { npu }|x| \leqslant 2,\end{cases}
$$

удовлетворяющее при $|x| \geqslant 1$ неравенству

$$
\Gamma_{\alpha_{0}}(x) \geqslant C|x|^{m}
$$

Доказательство лемм 10-12 будет дано в $\S 2$.

$5^{\circ}$. Стабилизации решений параболических уравнений без младших членов. Влияние начальных функций и областей. Вопросам, связанньп с условиями сушествования предела при $t \rightarrow \infty$ решени задачи Коши и смешанных задач в терминах свойств начальной функции $u_{0}(x)$ в случае, когда младшие коэффициенты уравнения не влияют на стабилизацию, посвяшено большое число работ.

Стабилизации решения Коши без младших членов с ограниченной или растушей начальной функцией посвящена обзорная статья [38].

Начало интересных исследований по стабилизации решения задачи Коши для уравнения теплопроводности положено в 50-х годах М. Кржижанским [23]. В частности, им построен пример ограниченной начальной функции, для которой решение задачи Коши

$$
\Delta u-u_{t}=0, \quad u(x, 0)=u_{0}(x)
$$

не имеет предела при $t \rightarrow \infty$. 
В работе [10] (см. также [60]) показано, что из оценок Нэша модуля непрерьвности решения и момента фундаментального решения уравнения

$$
\begin{gathered}
L(x, t) u-u_{t}=0, \\
u(x, 0)=u_{0}(x)
\end{gathered}
$$

вытекает достаточное условие существования равномерного в $\mathbb{R}^{N}$ предела (80), которое выражается в терминах сушествования равномерного по $x$ в $\mathbb{R}^{N}$ предела шарового среднего:

$$
\lim _{R \rightarrow \infty} \frac{N}{\omega_{N} R^{N}} \int_{|x-y| \leqslant R} u_{0}(y) \mathrm{d} y=A .
$$

В. Д. Репников и С. Д. Эйдельман [18] показали, что условие (99) в случае уравнения (97) с переменньми коэффициентами $a_{i k}(x, t)$ и ограниченной $u_{0}(x)$ является и необходимьм условием равномерной в $\mathbb{R}^{N}$ стабилизации решения задачи $(97),(98)$.

Доказательству этого критерия стабилизации решения задачи (96) посвящена работа В. Д. Репникова и С. Д. Эйдельмана [61].

В 1969 г. Ю.Н. Дрожжинов [62] установил, что такой же критерий стабилизации имеет место и для случая полуограниченных начальных функций из класса, которьй обеспечивает представление решения задачи Коши (96) в виде интеграла Пуассона.

В работе Ю.Н. Дрожжинова и Б.И. Завьялова [63] в качестве следствия из установленных ими теорем тауберова типа получен критерий существования квазипредела решения задачи (96) с непрерьвной начальной функцией $u_{0}(x)$ из класса Шварца $S^{1}\left(\mathbb{R}^{1}\right)$

При $\alpha>-1$ рассмотрим интегральные средние Чезаро-Рисса порядка $\alpha$ от начальной функщии $u_{0}(x)$ :

$$
S_{R}^{\alpha} u_{0}(x)=\frac{2}{B(N / 2, \alpha+1) \omega_{N} R^{N}} \int_{|x-y| \leqslant R} u_{0}(y)\left(1-\frac{|x-y|^{2}}{R^{2}}\right)^{\alpha} \mathrm{d} y .
$$

Во многих работах (см. библиографию в [38]) достаточные условия существования предела (80) решения задачи (97), (98) выражались в терминах сушествования предела

$$
\lim _{R \rightarrow \infty} S_{R}^{\alpha} u_{0}(x)
$$

средних (100).

Необходимое условие стабилизации равномерной в $\mathbb{R}^{1}$ стабилизации решения задачи Коши (96) с начальной функцией $u_{0}(x)$, удовлетворяюшей условию $(81)$, дает следующее утверждение, доказанное в [64].

ТеОрема [64]. Пусть $u_{0}(x), x \in \mathbb{R}^{1}$, удовлетворяет условию (81) и решение задачи (96) имеет предел (80) равномерно в $\mathbb{R}^{1}$, тогда существует предел

$$
\lim _{R \rightarrow \infty} \frac{n+1}{R} \int_{|x-y| \leqslant R}\left(1-\frac{|x-y|}{R}\right)^{n} u_{0}(y) \mathrm{d} y
$$

средних иелого порядка, $n \geqslant m$, равномерно по $x$ на каждом сегменте $[a, b] \subset \mathbb{R}^{1}$.

В [65] установлено более точное утверждение. 
ТЕОРема [65]. Если начальная функиия $u_{0}(x)$ непрерывна в $\mathbb{R}^{N}$ и удовлетворяет условию (81) и решение задачи Коши (96) имеет равномерный в $\mathbb{R}^{N}$ предел (80), то при

$$
\alpha>m-1
$$

существует предел (101) шаровых средних Чезаро-Рисса порядка а от и й , равномерный по $x$ на любом компакте $K \subset \mathbb{R}^{N}$.

$\mathrm{B}[65]$ доказано, что условие $\alpha>m-1$ является точным и не может быть заменено на $\alpha>m-1-\varepsilon, \varepsilon>0$.

В работе [66] (см. также [67]) установлено, что необходимое условие равномерной в $\mathbb{R}^{N}$ стабилизации решения задачи Коши (96) начальной функцией $u_{0}(x)$ из тихоновского класса нельзя выразить в терминах равномерной сходимости шаровых средних Чезаро-Рисса какого-либо порядка $\alpha \geqslant 0$, ибо, как доказано в [68], для любого $\alpha \geqslant 0$ сушествует непрерьвная в $\mathbb{R}^{N}$ начальная функция $u_{0}(x)$, удовлетворяюшая условию А.Н. Тихонова

$$
\left|u_{0}(x)\right| \leqslant C(\varepsilon) e^{\varepsilon|x|^{2}}
$$

и такая, что решение задачи Коши (96) не стабилизируется равномерно в $\mathbb{R}^{N}$, а ее шаровые средние Чезаро-Рисса (100) порядка $\alpha$ не имеют предела (101) ни в одной точке $x \in \mathbb{R}^{N}$.

Необходимые и достаточные условия поточечной стабилизации решени задачи Коши (96) с начальной функцией из класса А.Н. Тихонова установлены в работе В.Д. Репникова [29], и эти условия выражаются в терминах средних типа (101), когда порядок $\alpha(R)$ стремится к бесконечности при $R \rightarrow \infty$ согласованно с $C(\varepsilon)$, характеризующим класс функций (103) при $\varepsilon \rightarrow 0$.

Далее мы будем рассматривать ограниченные функции $u_{0}(x)$.

Случай простейшего уравнения с переменньми коэффициентами вида

$$
\Delta u-p(x) u_{t}=0, \quad u(x, 0)=u_{0}(x),
$$

рассмотрен в работах А.К. Гущина и В.П. Михайлова [57], [69].

В предположении, что

$$
\lim _{R \rightarrow \infty} R^{-N} \int_{|x-y| \leqslant R}\left|p(y)-p_{0}\right| \mathrm{d} y=0, \quad p_{0}>0,
$$

равномерно по $x \in \mathbb{R}^{N}$, в [69] доказано, что существование предела (99) в какой-либо точке $x \in \mathbb{R}^{N}$, а следовательно, и в каждой точке $x \in \mathbb{R}^{N}$ (равномерно на любом компакте $K$ в $\mathbb{R}^{N}$ ) необходимо и достаточно для стабилизации решения задачи (104) в этой точке (и равномерно на любом компакте $K \subset \mathbb{R}^{N}$ ).

В работах Е.Б. Сандакова [70], [71] аналогичные результаты были получены для параболического уравнения высокого порядка.

В работе $\Phi$. О. Порпера и С. Д. Эйдельмана [56] были получены результаты о стабилизации решения задачи Коши для одномерного уравнения

$$
\left(T(x)(S(x) u(x, t))_{x}\right)_{x}-q(x) u_{t}=0, \quad(x, t) \in \mathbb{R}_{+}^{2},
$$

которые являются весьма близкими к утверждениям работы А. К. Гушина и В. П. Михайлова [57], где изучалась задача Коши для одномерного параболического уравнения с ограниченной начальной функщией и были получены интересные результаты о необходимых и достаточных условиях стабилизашии решения задачи Коши. 
В работе $\Phi$. О. Порпера и С.Д. Эйдельмана [72] замечено, что при получении критерия равномерной в $\mathbb{R}^{1}$ стабилизации решения задачи Коши для уравнения (105) от условий на коэффищиенты можно освободиться. Это не относится к случаю теорем о близости решений при $t \rightarrow \infty$. Многомерньй случай изучен в [73].

В работах В. В. Жикова [19] и С. Л. Каменомостской [20] содержатся наиболее точные и полные результаты по стабилизации решения задачи Коши для параболических уравнений второго порядка без младших коэффициентов и с ограниченной начальной функцией $u_{0}(x) \in L^{\infty}\left(\mathbb{R}^{N}\right)$.

В них, в частности, доказано, что для равномерной стабилизации решения задачи Коши $(97),(98)$ с ограниченной в $\mathbb{R}^{N}$ начальной функцией необходимо и достаточно, чтобы сушествовал равномерный в $\mathbb{R}^{N}$ предел шарового среднего (99) от $u_{0}(x) \in$ $L^{\infty}\left(\mathbb{R}^{N}\right)$.

В предположении, что коэффишиенты $a_{i k}(x, t)$ удовлетворяют условию, что сушествуют пределы

$$
\lim _{R \rightarrow \infty} \frac{1}{R^{N+1}} \int_{0}^{R} \mathrm{~d} \tau \int_{|x| \leqslant R}\left[a_{i k}(x, \tau)-\widehat{a}_{i k}\right]^{2} \mathrm{~d} x=0,
$$

где $\widehat{a}_{i k}$ - положительно определенная матрица, в [20] доказано, что существование поточечного в $\mathbb{R}^{N}$ предела

$$
\lim _{R \rightarrow \infty} \frac{\int_{(B y, y) \leqslant R^{2}} u_{0}(x+y) \mathrm{d} y}{\int_{(B y, y) \leqslant R^{2}} \mathrm{~d} y}=l
$$

где $B$ - матрица, обратная матрице $\widehat{a}_{i k}$, необходимо и достаточно для существования поточечного в $\mathbb{R}^{N}$ предела (80).

Исследованию зависимости от неограниченной области $\Omega \subset \mathbb{R}^{N}$ коэффициентов уравнения без младших членов

$$
L(x, t) u-u_{t}=0, \quad(x, t) \in G=\Omega \times(0, \infty),
$$

удовлетворяющего условиям

$$
\left.\frac{\partial u}{\partial n}\right|_{\partial \Omega}=0, \quad t>0, \quad u(x, 0)=u_{0}(x), \quad x \in \Omega,
$$

посвящены работа А. К. Гушина, В.П. Михайлова и Ю.А. Михайлова [26] и работы А. К. Гушина [27], [74]-[76].

В работах А.К. Гущина [27], [74], [75] решена следуюшая задача: найти функшию $U(t), t>0$, и такой класс начальных функщий $u_{0}(x)$, что при каждой функщии $u_{0}(x)$ из этого класса решение задачи (107), (108) стремится к нулю не медленнее функции $U(t)$, т.е.

$$
\|u(\cdot, t)\|_{L^{\infty}(\Omega)} \leqslant C U(t),
$$

и для любой неотрицательной начальной функции $u_{0}(x) \not \equiv 0$ решение стремится к нулю не быстрее функции $U(t)$, т.е.

$$
\|u(\cdot, t)\|_{L^{\infty}(\Omega)} \geqslant C_{u_{0}} U(t),
$$


где $C=C_{u_{0}}>0$ зависит от $u_{0}$.

Установлено [74], что для широкого класса неограниченных областей максимальная скорость стабилизации задачи (107)-(109) определяется функцией

$$
V(R)=\operatorname{mes} \Omega(R, 0),
$$

где

$$
\Omega(R, x)=\{\xi \in \Omega:|\xi-x| \leqslant R\},
$$

и в качестве функции $U(t)$ в оценках (109) и (110) можно взять функцию

$$
U(t)=\frac{1}{V(\sqrt{t})} .
$$

Можно считать, что начало координат $O \in \Omega$. От коэффициентов уравнения зависят лишш постоянные $C$ и $C_{u_{0}}$ в оценках (109) и (110).

Для областей $\Omega$, “не сужаюшихся на бесконечности” (т.е., грубо говоря, таких, из которых можно выкатить на бесконечность шар некоторого радиуса), классом начальных функций, обеспечивающим максимальную скорость стабилизации, определяемую из неравенств (109) и $(110)$, является пространство $L_{1}(\Omega)[74]$, и постоянная в (110) имеет вид $C_{0}\left\|u_{0}\right\|_{L_{1}(\Omega)}$. Условие $u_{0} \in L_{1}(\Omega)$ неулучшаемо, если $u_{0}(x) \geqslant 0$ [28], [75].

Для “сужаюшихся” областей, для которых $\lim V(r) / r=0$, максимальньй порядок стремления решения к нулю обеспечивается условием

$$
\int_{\Omega}|x|\left|u_{0}(x)\right| \mathrm{d} x<\infty
$$

на начальную функцию [27].

В работе А.К. Гушина [76] установлен критерий равномерной в области $\Omega \subset \mathbb{R}^{N}$ стабилизации решения второй смешанной задачи $(107),(108)$. При условиях, что $u_{0}(x) \in L^{\infty}\left(\mathbb{R}^{N}\right), \Omega \in \mathscr{U}\left(a V^{(n-1) / n}\right)$ (см. [76]), этот критерий является близким к соответствуюшему критерию для задачи Коши [19].

Теорема [76]. Для равномерной относительно $x$ из $\Omega \subset \mathbb{R}^{N}$ стабилизации решени задачи (107), (108), т.е. для существования предела

$$
\lim _{t \rightarrow \infty} u(x, t)=l \quad \text { равномерно по } x \text { из } \Omega,
$$

необходимо и достаточно, чтобь

$$
\lim _{R \rightarrow \infty} \frac{1}{V(x, R)} \int_{\Omega(x, R)} u_{0}(y) \mathrm{d} y=l \quad \text { равномерно по } x \text { из } \Omega, V(x, R)=\operatorname{mes} \Omega(R, x) .
$$

Вопросы стабилизации решения первой смешанной задачи

$$
\begin{gathered}
L(x, t) u-u_{t}=0 \text { в } \quad D=\Omega \times(0, \infty), \\
\left.u\right|_{\partial \Omega}=0, \quad t>0, \\
\left.u\right|_{t=0}=u_{0}(x), \quad x \in \Omega,
\end{gathered}
$$

были изучены в работах $[21],[22],[25],[47]$, [48] и в работах [77], [78]. 
Наиболее сильньй результат получен в работе [25] Ф.Х. Мукминова. В предположении, что неограниченная область $\Omega \subset \mathbb{R}^{N}$ удовлетворяет условию: сушествует постоянная $\theta>0$ такая, что для всякой точки $x$ гранишы $\partial \Omega$ справедливо неравенство

$$
\operatorname{mes}(\{y:|x-y|<r\} \backslash \Omega) \geqslant \theta r^{N}, \quad r>0,
$$

и для некоторого класса начальных функций, включающего в себя все ограниченные функции $u_{0}(x)$, в работе $\Phi . Х$. Мукминова [25] установлено, что необходимым и достаточным условием равномерной стабилизации к нулю решения задачи (111)-(113) является следуюшее условие:

$$
\lim _{R \rightarrow \infty} R^{-N} \int_{|x-y|<R} u_{0}(y) \mathrm{d} y=0
$$

равномерно по $x$ из $\Omega$ таким, что $\operatorname{dist}(x, \partial \Omega) \geqslant R+1$.

Доказательство опирается на полученную в [25] оценку функции Грина.

Вопросы стабилизации решения третьей смешанной задачи для параболического уравнения второго порядка

$$
\begin{gathered}
L(x, t) u-u_{t}=0 \text { в } \quad G=\Omega \times(0, \infty), \\
\left.\left(\sum_{i, k=1}^{N} a_{i k} \frac{\partial u}{\partial x_{i}} \nu_{k}-\nu_{0} u\right)\right|_{(x, t) \in \Gamma}=0, \\
u(x, 0)=u_{0}(x), \quad x \in \Omega,
\end{gathered}
$$

были изучены в работах [79]-[81], [14], где $\Gamma=\partial G \cup\{t>0\}, \nu=\left(\nu_{0}, \ldots, \nu_{N}\right)-$ вектор внешней к Г нормали и $D_{0}-$ проекция сечения области $\Omega$ гиперплоскостью $t=0$.

Наиболее сильные результаты получены в работе В. Г. Ушакова [81], в которой исследовано асимптотическое поведение при $t \rightarrow \infty$ решения третьей смешанной задачи (114)-(116) в нецилиндрической области. Эти результаты аналогичны по форме сформулированному вьше результату А. К. Гущина [74], т.е. справедливы оценки (109) и (110).

\section{§. Некоторые свойства решений эллиптических уравнений}

Здесь мы докажем леммы 10-12, сформулированные в п. $4^{\circ} \S 1$, и другие необходимые факты о решениях эллиптических уравнений.

$\mathrm{B} \mathbb{R}^{N}$ рассмотрим эллиптическое уравнение

$$
\sum_{i, k=1}^{N}\left(a_{i k}(x) \Gamma_{x_{k}}\right)_{x_{i}}+\sum_{i=1}^{N} b_{i}(x) \Gamma_{x_{i}}+q(x) \Gamma=0,
$$

или, кратко,

$$
L(x) \Gamma+(b(x), \nabla \Gamma)+q(x) \Gamma=0,
$$

где $a_{i k}(x)=a_{k i}(x)$, вьполнено условие $(2,1)$ эллиптичности, коэффициенты уравнения (1) измеримы и ограничены в $\mathbb{R}^{N}$. Пусть при $N=1$ или $N=2$ коэффициенты $b_{i}(x)$ удовлетворяет условиям $\left(\mathrm{B}_{1}\right)$, а коэффициент $q(x)$ определен равенством

$$
q(x)= \begin{cases}-\alpha^{2} & \text { при }|x|<1, \\ 0 & \text { при }|x| \geqslant 1 .\end{cases}
$$


Напомним [40], что под решением уравнения (1) в $\mathbb{R}^{N}$ мы понимаем такую функцию $\Gamma(x)$, которая принадлежит классу $W_{2}^{1, \operatorname{loc}}\left(\mathbb{R}^{N}\right)$ и при любой пробной функции $\varphi(x) \in$ $C_{0}^{\infty}\left(\mathbb{R}^{N}\right)$ удовлетворяет интегральному тождеству

$$
\int_{\mathbb{R}^{N}}[(a(x) \nabla \Gamma, \nabla \varphi)-\varphi((\nabla \Gamma, b)+\Gamma q)] \mathrm{d} x=0 .
$$

Докажем лемму 10 при $N=2$, ибо в случае $N=1$ доказательство проводится аналогично, но требует отдельной записи.

ДокАЗАТЕЛЬСТво ЛЕмМЫ 10 будем подразделять на отдельные пункты.

$1^{\circ}$. Докажем, что сушествует ограниченное и положительное в $\mathbb{R}^{2}$ решение уравнения (1).

Пусть $B_{n}=\{x:|x|<n\}, n \in \mathbb{N},-$ последовательность кругов в $\mathbb{R}^{2}$. Пусть фиксировано некоторое $M>0$. В каждом круге $B_{n}$ рассмотрим задачу

$$
\begin{gathered}
L(x) V_{n}+\left(b(x), \nabla V_{n}\right)+q(x) V_{n}=0 \quad \text { в } \quad B_{n}, \quad n \in \mathbb{N}, \\
\left.V_{n}\right|_{|x|=n}=M .
\end{gathered}
$$

Известно [40], [82], что при каждом $n$ решение задачи (4), (5) сушествует и единственно. Из усиленного принципа максимума [82] вытекает, что для $V_{n}(x)$ справедливы неравенства

$$
0<V_{n}(x)<M
$$

и что семейство функций $\left\{V_{n}(x)\right\}, n \in \mathbb{N}$, является невозрастаюшим: $V_{n_{2}} \leqslant V_{n_{1}}$ при $n_{2}>n_{1}, x \in B_{n}$.

Из оценок Э. Де Джорджи (см. [40], [82]) следует, что $V_{n}(x)$ удовлетворяет условию Гёльдера равномерно по $x$ в каждом круге $B_{r}, r<n$. Это означает, что семейство функций $\left\{V_{n}(x)\right\}$ удовлетворяет лемме Арцела [83] на каждом компакте $K$ в $B_{n}$. Применяя диагональньй процесс, получим, что сушествует подпоследовательность $\left\{V_{n_{k}}(x)\right\}$ последовательности $\left\{V_{n}(x)\right\}$, которая сходится в $\mathbb{R}^{2}$ к некоторой функции $V(x)$, причем сходимость является равномерной на каждом компакте $K$ в $\mathbb{R}^{2}$. Из этого следует, что $V_{n_{k}}(x) \underset{n_{k} \rightarrow \infty}{\longrightarrow} V(x)$ в $L^{2}\left(B_{r}\right)$ при каждом $r>0$.

Из оценки [84]

$$
\int_{B_{r_{1}}}\left|\nabla V_{n_{k}}\right|^{2} \mathrm{~d} x \leqslant c\left(r_{1}, r_{2}\right) \int_{B_{r_{2}}}\left|V_{n_{k}}\right|^{2} \mathrm{~d} x, \quad 0<r_{1}<r_{2},
$$

следует, что существует слабо сходящаяся в $W_{2}^{1, \text { lос }}\left(\mathbb{R}^{2}\right)$ подпоследовательность:

$$
V_{n_{k_{i}}}(x) \underset{n_{k_{i}} \rightarrow \infty}{\rightarrow} V(x) \text { слабо в } W_{2}^{1, \text { loc }}\left(\mathbb{R}^{2}\right) .
$$

Отсюда легко следует, что предельная функция $V(x)$ является обобщенным $W_{2}^{1, \text { loc }}\left(\mathbb{R}^{2}\right)$-решением уравнения (1), для которого справедливы неравенства

$$
0 \leqslant V(x) \leqslant M \text {. }
$$

$2^{\circ}$. Докажем, что построенное в п. $1^{\circ}$ решение $V(x)$ таково, что сушествует предел

$$
\lim _{|x| \rightarrow \infty} V(x)=m \leqslant M .
$$

Рассмотрим кольцо $S_{\lambda}=\left\{x \in \mathbb{R}^{2}: \lambda / 2<|x|<2 \lambda\right\}, \lambda>2$, и воспользуемся следуюшим известным утверждением. 
ЛЕмма 13 (О.А. Ладыженская; см. [40], [82]). Eсли $V(x) \geqslant 0$ - любое ограниченное решение уравнения

$$
L(x) V+(b, \nabla V)=0, \quad|x|<1,
$$

то существует $\delta, 0<\delta<1$, для которого

$$
\operatorname{osc}_{|x|<1 / 2} V(x) \leqslant \delta \cdot \operatorname{osc}_{|x|<1} V(x) .
$$

$\left[3 \partial e c b \operatorname{osc}_{B_{r}} V=\sup _{B_{r}} V(x)-\inf _{B_{r}} V(x)\right.$.]

Из этой леммы и неравенства (8) следует, что

$$
\operatorname{osc}_{|x|=\lambda} V(x) \leqslant \delta \cdot \operatorname{osc}_{\lambda / 2<|x|<2 \lambda} V(x) \leqslant \delta \cdot 2 M
$$

Применяя внешний принцип максимума Дж. Серрина [85] к функции $V(x)$ в области $|x|>\lambda$, будем иметь

$$
\operatorname{osc}_{|x|>\lambda} V(x) \leqslant \delta \cdot 2 M .
$$

Для удобства читателя напомним принцип максимума.

Лемма 14 Дж. Серрин [85]. Если $N=2$ и $V(x)$ - ограниченное решение $V(x)$ уравнения (10) при $|x|>\lambda-$-удвлетворяет неравенству $\left.V(x)\right|_{|x|=\lambda} \leqslant M$, то $V(x) \leqslant M n p u|x|>\lambda .^{2}$

Рассмотрим кольцо $S_{2 \lambda}=\left\{x \in \mathbb{R}^{2}: \lambda<|x|<4 \lambda\right\}$, применим лемму 13 О.А. Ладыженской и неравенство (12), при этом получим

$$
\operatorname{osc}_{|x|=2 \lambda} V(x) \leqslant \delta \operatorname{osc}_{\lambda<|x|<4 \lambda} V(x) \leqslant 2 M \delta^{2} .
$$

Из леммы 14 тогда следует, что

$$
\operatorname{osc}_{|x|>2 \lambda} \leqslant 2 M \delta^{2} .
$$

Далее по индукщии легко получаем, что

$$
\operatorname{osc}_{|x|>2^{k} \lambda} V(x)<2 M \delta^{k+1}, \quad 0<\delta<1 \quad(k=1,2, \ldots) .
$$

Из неравенства (13) и критерия Коши сушествования предела [83] получим, что существует предел (9).

$3^{\circ}$. Докажем, что предел (9) равен нулю, т.е. что $m=0$.

Вначале установим, что не сушествует точек $x_{0}$ из $\mathbb{R}^{2}$, для которых $V\left(x_{0}\right)>m$.

Предположим противное, т.е. что существует $x_{0} \in \mathbb{R}^{2}$, для которой

$$
V\left(x_{0}\right)>m \text {. }
$$

Положим $\varepsilon=\left(V\left(x_{0}\right)-m\right) / 2>0$. В силу существования предела (9) для выбранного $\varepsilon>0$ найдется $R$ такое, что при $|x| \geqslant R$ справедливо неравенство

$$
-\varepsilon<V(x)-m<\varepsilon,
$$

\footnotetext{
${ }^{2} \mathrm{~B}[85]$ рассматриваются классические решения. Легко видеть, что лемма 14 справедлива для решений уравнения $(10)$ из $W_{2}^{1, \text { loc }}\left(\mathbb{R}^{2}\right)$ в смысле интегрального тождества (3).
} 
т.е. неравенство

$$
V(x)<\frac{V\left(x_{0}\right)-m}{2}+m=\frac{V\left(x_{0}\right)+m}{2}<V\left(x_{0}\right), \quad|x| \geqslant R .
$$

Выберем натуральное $k$ так, чтобы точка $x_{0}$, в которой $V\left(x_{0}\right)>m$, попала внутрь круга $B_{k}$ радиуса $k R$. На границе круга $B_{k}$ в силу $(15)$ справедливо неравенство

$$
\left.V(x)\right|_{|x|=k R}<V\left(x_{0}\right) .
$$

По теореме Вейерштрасса [83] непрерывная в замкнутом круге $\bar{B}_{k R}$ функция $V(x)$ достигает максимального значения. В силу (16) максимум $V(x)$ не может достигаться на гранище $|x|=k R$, следовательно, максимальное значение $V(x)$ достигается во внутренней точке круга $B_{k}$. Это приводит к противоречию, ибо, согласно принципу максимума [40], [82], любое решение уравнения (1) в круге $\bar{B}_{k R}$ при $q(x) \leqslant 0$ достигает своего максимального значения на границе этого круга. Следовательно, доказано, что $V(x)$ удовлетворяет неравенству $V(x) \leqslant m, x \in \mathbb{R}^{N}$.

Из усиленного принципа максимума Хопфа [82] тогда следует, что

$$
V(x)<m \text { для всех } x \in \mathbb{R}^{2} .
$$

Рассмотрим кольцо $\bar{S}_{2 \lambda}=\left\{x \in \mathbb{R}^{2}: \lambda \leqslant|x| \leqslant 4 \lambda\right\}$. Тогда для всех $x \in \bar{S}_{2 \lambda}$ в силу (17) имеем $m-V(x)>0$. По теореме Вейерштрасса [83] непрерывная в $\bar{S}_{2 \lambda}$ функция $h(x)=1 /(m-V(x))$ является ограниченной в этом кольце, т.е. сушествует постоянная $M_{1}>0$, для которой

$$
h(x) \leqslant M_{1}, \quad x \in \bar{S}_{2 \lambda} .
$$

Последнее неравенство может быть переписано так:

$$
V(x) \leqslant m-\frac{1}{M_{1}}, \quad x \in \bar{S}_{2 \lambda} .
$$

Применяя лемму 14 [85] к решению $V(x)$ уравнения (1) во внешности круга $B_{4 \lambda}$, получим в силу (18) неравенство

$$
V(x) \leqslant m-\frac{1}{M_{1}}, \quad|x|>4 \lambda, \quad M_{1}>0 .
$$

Неравенство (19) приводит к противоречию с сушествованием предела (9), равного $m$.

Итак, доказано, что не сушествует точек $x$ из $\mathbb{R}^{2}$, для которых имеют место неравенства (14) и (17). Следовательно, доказано, что $V(x) \equiv m=$ const, $x \in \mathbb{R}^{2}$, где $m$ предел (9). Но $V(x)$ является решением уравнения (1) и ясно, что среди всех констант $m$ только $m=0$ является решением уравнения (1). Из принципа максимума [40], [82] тогда имеем $V \equiv 0$ в $\mathbb{R}^{2}$.

$4^{\circ}$. Докажем, что сушествует решение $\Gamma(x)$ уравнения $(1)$, удовлетворяюшее условиям 1)-3) леммы 10. Рассмотрим семейство функций

$$
\Gamma_{n}(x)=\frac{V_{n}(x)}{V_{n}(0)} \quad(n=1,2, \ldots)
$$


где $V_{n}(x)$ - решение задачи (4), (5). Ясно, что при каждом $n \in \mathbb{N}$ функция $\Gamma_{n}(x)$ удовлетворяет уравнению (4) в круге $B_{n}$ и $\Gamma_{n}(0)=1$. Применим к $\Gamma_{n}(x)$ неравенство Харнака [82], справедливое для любого неотрицательного решения уравнения (4):

$$
\sup _{B_{r}} \Gamma_{n}(x) \leqslant C_{1} \inf _{B_{r}} \Gamma_{n}(x)
$$

и, учитывая, что $\Gamma_{n}(0)=1$, получим неравенство

$$
\sup _{|x| \leqslant r} \Gamma_{n}(x) \leqslant C
$$

в котором постоянная $C$ зависит от $N, l, r$, но не зависит от $n$. Таким образом, семейство функций (20) является компактньм относительно равномерной сходимости на компакте $K$ в $\mathbb{R}^{2}$.

Применяя диагональный процесс, можно выделить из $\left\{\Gamma_{n}(x)\right\}$ подпоследовательность $\left\{\Gamma_{n_{k}}(x)\right\}$, которая сходится при $n_{k} \rightarrow \infty$ к некоторой функции $\Gamma(x)$ равномерно по $x$ на каждом компакте $K$ в $\mathbb{R}^{2}$. Отсюда следует, что

$$
\Gamma_{n_{k}}(x) \underset{n_{k} \rightarrow \infty}{\longrightarrow} \Gamma(x) \text { в } L^{2}\left(B_{r}\right), \quad r>0 .
$$

Из оценки (7) тогда получим, что существует подпоследовательность $\left\{\Gamma_{n_{k_{i}}}(x)\right\}$, для краткости ее снова обозначим $\Gamma_{n_{k}}(x)$, которая слабо в $W_{2}^{1, \text { lос }}\left(\mathbb{R}^{2}\right)$ сходится к $\Gamma(x)$. Отсюда легко следует, что $\Gamma(x)$ является обобшением решения уравнения $(1)$ в смысле интегрального тождества (3) и при этом $\Gamma(0)=1, \Gamma(x)>0, x \in \mathbb{R}^{2}$.

$5^{\circ}$. Докажем, что построенное в п. $4^{\circ}$ решение $\Gamma(x)$ уравнения $(1)$ таково, что

$$
\lim _{|x| \rightarrow \infty} \Gamma(x)=+\infty
$$

Вначале докажем, что функция $\Gamma(x)$ является неограниченной в $\mathbb{R}^{2}$. Предположим противное, тогда сушествует постоянная $M>0$, для которой $0 \leqslant \Gamma(x) \leqslant M, x \in \mathbb{R}^{2}$. Применяя для $\Gamma(x)$ построения п. $2^{\circ}$ и $3^{\circ}$, мы аналогично предыдушему докажем, что сушествует предел

$$
\lim _{|x| \rightarrow \infty} \Gamma(x)=0
$$

и, следовательно, из принципа максимума [82] получим, что $\Gamma(x) \equiv 0, x \in \mathbb{R}^{2}$. Получим противоречие, ибо $\Gamma(0)=1$. Полученное противоречие доказьвает, что $\Gamma(x)$ является неограниченной в $\mathbb{R}^{2}$, т.е. для каждого $n \in \mathbb{N}$ сушествует точка $x_{n} \in \mathbb{R}^{2}$, в которой

$$
\left.\Gamma(x)\right|_{x=x_{n}} \geqslant n \text {. }
$$

Теперь докажем (22). Пусть (22) неверно. Тогда существуют постоянная $A$ и последовательность $y_{n},\left|y_{n}\right| \rightarrow \infty$ при $n \rightarrow \infty$, для которой

$$
\left.\Gamma(x)\right|_{x=y_{n}} \leqslant A \text { при } n \geqslant N(A) .
$$

Используя неравенство (24), тот факт, что функция Г $(x)$ является решением уравнения

$$
L(x) \Gamma+(b, \nabla \Gamma)=0 \text { при }|x|>1,
$$


и неравенство Харнака (21), легко доказать, что существует постоянна $C_{2}>0$, для которой

$$
\left.\Gamma(x)\right|_{|x|=\left|y_{n}\right|} \leqslant C_{2} A, \quad n \geqslant N(A) .
$$

Действительно, пусть $n \geqslant N(A)$. Обозначим $\lambda_{n}=\left|y_{n}\right|$, построим в кольце $S_{\lambda_{n}}=$ $\left\{x \in \mathbb{R}^{2}: \lambda_{n} / 2<|x|<2 \lambda_{n}\right\}$ окружность $|x|=\lambda_{n}$ и заменим в уравнении (25) переменные по формулам $x_{i}=\frac{\lambda_{n}}{4} \sigma_{i}(i=1,2)$. Тогда кольцо $\bar{S}_{\lambda_{n}}$ перейдет в кольцо $2 \leqslant|\sigma| \leqslant 8$, окружность $|x|=\lambda_{n}$-в окружность $|\sigma|=4$, уравнение $(25)$ - в уравнение

$$
\sum_{i, k=1}^{N} \frac{\partial}{\partial \sigma_{i}}\left(a_{i k}\left(\frac{\lambda_{n}}{4} \sigma\right) \frac{\partial W}{\partial \sigma_{k}}\right)+\sum_{k=1}^{N} \frac{\lambda_{n}}{4} b_{k}\left(\frac{\lambda_{n}}{4} \sigma\right) \frac{\partial W}{\partial \sigma_{k}}=0 \quad \text { в } 2<|\sigma|<8
$$

где $W=\Gamma\left(\frac{\lambda_{n}}{4} \sigma\right)$.

Из условий $(2,1)$ равномерной эллиптичности и условий $\left(\mathrm{B}_{1}\right)$ легко следует, что коэффициенты преобразованного уравнения (27) имеют в кольце $2<|\sigma|<8$ верхние грани, не зависяшие от $\lambda_{n}$. Поэтому оценки для решений $W$ в кольце $2<|y|<8$ уравнения (27) будут справедливы с теми же постоянньми и в кольце $S_{\lambda_{n}}$ для решений $\Gamma(x)$ уравнения (25). Рассмотрим окружность $|\sigma|=4$. Некоторая точка $\sigma_{1}$ этой окружности является образом точки $y_{n}(24)$ при отображении $\sigma_{i}=\frac{4}{\left|y_{n}\right|} x_{i},|x|=\left|y_{n}\right|$. Впишем в кольцо $2 \leqslant|\sigma| \leqslant 8$ замкнутьй круг $\bar{B}_{1}^{\sigma_{1}}$ с центром в точке $\sigma_{1}$ и радиуса 1 и применим неравенство Харнака (21). В результате получим

$$
\sup _{\bar{B}_{1}^{\sigma_{1}}} W \leqslant C_{1} W\left(\sigma_{1}\right) \leqslant C_{1} A .
$$

Круг $B_{1}^{\sigma_{1}}$ пересекает окружность $|\sigma|=4$ в двух точках $\sigma_{2}^{\prime}$ и $\sigma_{2}^{\prime \prime}$. Возьмем любую из этих точек (например, $\sigma_{2}^{\prime}$ ) и впишем в кольцо $2 \leqslant|\sigma| \leqslant 4$ круг $\bar{B}_{1}^{\sigma_{2}^{\prime}}$ с центром в точке $\sigma_{2}^{\prime}$ радиуса 1 . Применяя неравенство Харнака $(21)$ в $B_{1}^{\sigma_{2}^{\prime}}$ и затем неравенство $(28)$, получим

$$
\sup _{\bar{B}_{1}^{\sigma_{2}^{\prime}}} W \leqslant C_{1} \inf _{\bar{B}_{1}^{\sigma_{2}}} W \leqslant C_{1}^{2} A
$$

Круг $B_{1}^{\sigma_{2}^{\prime}}$ пересекает окружность $|\sigma|=4$ в двух точках $\sigma_{3}^{\prime}$ и $\sigma_{3}^{\prime \prime}$, одна из которых (пусть это будет $\sigma_{3}^{\prime}$ ) не принадлежит кругу $B_{1}^{\sigma_{1}}$. Впишем круг $B_{1}^{\sigma_{3}^{\prime}},\left|\sigma_{3}^{\prime}\right|=4$, и, применяя неравенство Харнака $(21)$ в $\bar{B}_{1}^{\sigma_{3}^{\prime}}$, а затем неравенство $(29)$, получим

$$
\sup _{\bar{B}_{1}^{\sigma_{3}^{\prime}}} W \leqslant C_{1} \inf _{\bar{B}_{1}^{\sigma_{3}^{\prime}}} W \leqslant C_{1}^{3} A .
$$

Продолжая далее процесс построения вписанных кругов с центрами на окружности $|\sigma|=4$ и радиуса 1 , мы покроем окружность $|\sigma|=4$ конечной цепью $k_{0}$ кругов радиуса 1 и получим неравенство

$$
\sup _{|\sigma|=4} W \leqslant C_{1}^{k_{0}} A, \quad k_{0}=32 \pi .
$$


Совершая обратньй переход к переменным $x_{i}$ от переменных $\sigma_{i}: \sigma_{i}=\frac{4}{\lambda_{n}} x_{i}$, получим, что неравенство (26) доказано. При этом $C_{2}=C_{1}^{k_{0}}$.

Учитывая справедливость неравенства (26) при $|x|=\left|y_{k}\right|$ и применяя в области $|x|>\left|y_{n}\right|$ лемму 14 (внешний принцип максимума [85]), получим, что

$$
\Gamma(x) \leqslant C_{2} A \text { для }|x| \geqslant\left|y_{n}\right| \text {. }
$$

Получили противоречие, ибо, как доказано вьше, в силу (23) функци $\Gamma(x)$ является неограниченной. Следовательно, (22) доказано.

Лемма 10 доказана.

Для доказательства леммы 11 нам потребуется следующее утверждение, имеющее и самостоятельный интерес.

ЛЕмма 15. Если $и(x) \geqslant 0$ - решение уравнения

$$
L(x) u+(b(x), \nabla u)+q(x) u=0, \quad|x|<1, \quad q(x) \leqslant q_{0}<-0,
$$

удовлетворяющее условию

$$
\left.u\right|_{|x|=1}=M>0
$$

то существует $\delta(0<\delta<1)$, для которого

$$
u(0) \leqslant M \delta
$$

Для случая, когда $b_{i}(x)=0$, доказательство леммы 15 можно найти в [34], и оно основьвается только на оценках функции Грина задачи Дирихле в единичном шаре для уравнения без младших членов: $L(x) u=0,|x|<1$, приведенных в работе [86].

Для функции Грина задачи Дирихле в единичном шаре для уравнения $L u+(b, \nabla u)$ $=0$ точно такие же оценки получены в работе Г. Стампаккья [84]. Поэтому мы не будем доказьвать лемму 15, отсылая читателя к работе [34].

ЛЕмма 16. Пусть $и(x) \geqslant 0$ - любое решение уравнения (1) в кольце $S_{\lambda}=\left\{x \in \mathbb{R}^{N}: \lambda / 2<|x|<2 \lambda\right\}, \lambda>2$, əде

$$
q(x)= \begin{cases}-\frac{\alpha^{2}}{|x|^{2}} & \text { npu }|x|>1, \\ 0 & n p u|x| \leqslant 1,\end{cases}
$$

а для $b_{i}(x)$ выполнено условие $\left(\mathrm{B}_{2}\right)$, и пусть и $(x)$ удовлетворяет неравенствам

$$
\left.u\right|_{|x|=2 \lambda} \leqslant M,\left.\quad u\right|_{|x|=\lambda / 2} \leqslant M, \quad M>0 .
$$

Тогда существует $\delta(0<\delta<1)$, для которого

$$
\left.u\right|_{|x|=\lambda} \leqslant M \delta
$$


ДокАЗАТЕльство. В уравнении (1) сделаем замену переменных $x_{i}=\frac{\lambda}{4} y_{i}(i=$ $1, \ldots, N)$. Тогда уравнение (1) перейдет в уравнение

$$
L\left(\frac{\lambda}{4} y\right) V+\frac{\lambda}{4}\left(b\left(\frac{\lambda}{4} y\right), \nabla V\right)+\frac{\lambda^{2}}{4^{2}} q\left(\frac{\lambda}{4} y\right) V=0, \quad 2<|y|<8
$$

где $V=\Gamma\left(\frac{\lambda}{4} y\right)$.

Учтем, что в силу условий эллиптичности $(2,1)$ и условий $\left(\mathrm{B}_{2}\right)$ на $b_{k}(x)(k=1$, $\ldots, N)$ коэффициенты преобразованного уравнения (37) имеют верхние грани в слое $2 \leqslant|y| \leqslant 8$, не зависяшие от $\lambda$. Применяя лемму 15 к решению $V$ уравнения $(37)$ в шаре $B_{1}^{y}$ с центром в любой точке $y$ такой, что $|y|=4$, получим, что сушествует $\delta$ $(0<\delta<1)$, для которого

$$
V(y) \leqslant \delta M, \quad|y|=4 .
$$

Из произвольности $y \in\{|y|=4\}$ следует, что

$$
\sup _{|y|=4} V(y) \leqslant \delta M, \quad 0<\delta<1
$$

Возврашаясь к переменным $x$, получим, что требуемое неравенство доказано.

Лемма 16 доказана.

ДОКАЗАТЕЛЬСТВО ЛЕММЫ 11 ИЗ П. $4^{\circ} \S 1$.

$1^{\circ}$. Докажем, что сушествует ограниченное решение из $W_{2}^{1, \operatorname{loc}}\left(\mathbb{R}^{N}\right)$ уравнения (1), где коэффициент $q(x)$ определен по формуле $(36)$, а коэффищиенты $b_{i}(x)$ удовлетворяют условию $\left(\mathrm{B}_{2}\right)$.

$\mathrm{B}$ шаре $B_{n}=\left\{x \in \mathbb{R}^{N}:|x|<n\right\}, n \in \mathbb{N}$, рассмотрим уравнение

$$
L(x) V_{n}+\left(b(x), \nabla V_{n}\right)+q(x) V_{n}=0, \quad x \in B_{n},
$$

удовлетворяющее условию

$$
\left.V_{n}(x)\right|_{|x|=n}=M, \quad M>0
$$

Известно, что при каждом $n \in \mathbb{N}$ решение $V_{n}(x)$ задачи $(38),(39)$ сушествует и удовлетворяет неравенствам

$$
0<V_{n}(x) \leqslant M
$$

Из оценок Де Джорджи-Нэша (см. [40], [82]) решений линейных эллиптических уравнений следует, что $V_{n}(x)$ удовлетворяет условию Гёльдера равномерно в каждой области $\{|x|<n\}$. Это означает, что семейство $\left\{V_{n}(x)\right\}$ удовлетворяет лемме Арцела [83] на каждом компакте $K$ в области $\{|x|<n\}$.

Следовательно, сушествует подпоследовательность последовательности $\left\{V_{n}(x)\right\}$, которая равномерно по $x$ сходится на каждом компакте $K$ в $|x|<n, n \in \mathbb{N}$. Применяя диагональный процесс, получим, что существует подпоследовательность $\left\{V_{n_{k}}(x)\right\}$, которая сходится к некоторой функции $V(x)$, причем сходимость является равномерной на каждом компакте $K$ в $\mathbb{R}^{N}$. 
Из этого следует, что $V_{n_{k}}(x) \rightarrow V(x)$ в $L^{2}\left(B_{r}\right)$ при $n_{k} \rightarrow \infty$ для каждого $r>0$. Из оценки (7) вытекает, что сушествует подпоследовательность $\left\{V_{n_{k_{i}}}(x)\right\}$, которая сходится к $V(x)$ слабо в $W_{2}^{1}\left(B_{r}\right)$ при каждом $r>0$. Отсюда легко следует, что функция $V(x)$ является обобшенным решением уравнения $(1)$ в $\mathbb{R}^{N}$ и

$$
0<V(x)<M \text {. }
$$

$2^{\circ}$. Применяя лемму 16, докажем, что решение $V(x)$ уравнения $(1)$, построенное в предыдушем п. $1^{\circ}$, имеет предел

$$
\lim _{|x| \rightarrow \infty} V(x)=0 .
$$

Предположим, что $\lim _{|x| \rightarrow \infty} V(x) \neq 0$, и так как $V(x)>0$, то $V(x) \geqslant \gamma>0$. Обозначим

$$
\beta=\varlimsup_{|x| \rightarrow \infty} V(x) \geqslant \gamma>0 .
$$

Ясно, что верхний предел всегда существует.

По определению верхнего предела имеем

$$
V(x) \leqslant \beta, \quad x \in \mathbb{R}^{2} .
$$

Поэтому

$$
\left.V(x)\right|_{|x|=\lambda / 2} \leqslant \beta,\left.\quad V(x)\right|_{|x|=2 \lambda} \leqslant \beta .
$$

По лемме 16 тогда имеем

$$
\left.V\right|_{|x|=\lambda}<\delta \beta, \quad \text { где } 0<\delta<1,
$$

HO

$$
\beta=\varlimsup_{|x| \rightarrow \infty} V(x) \leqslant \delta \beta .
$$

Полученное противоречие доказьвает (41).

Из принципа максимума [82] и неравенства Харнака [82]

$$
\sup _{|x|=\lambda} V \leqslant C \inf _{|x|=\lambda} V<\varepsilon C,
$$

тогда легко получим, что $V(x) \equiv 0$ в $\mathbb{R}^{N}$.

$3^{\circ}$. Докажем, что сушествует решение $\Gamma(x)$ уравнения $(1)$, удовлетворяюшее условиям 1)-3) леммы 11.

Зафиксируем $x_{0},\left|x_{0}\right|>1$, и рассмотрим семейство функций

$$
\Gamma_{n}(x)=\frac{V_{n}(x)}{V_{n}\left(x_{0}\right)}, \quad\left|x_{0}\right|>1,
$$

где $V_{n}(x)$ - решение задачи $(38),(39)$, построенное в п. $1^{\circ}$.

Ясно, что $\Gamma\left(x_{0}\right)=1$ и функщия $\Gamma_{n}(x)$ является решением уравнения $(38)$ в $B_{n}$. Применяя неравенство Харнака [82], будем иметь

$$
\sup _{B_{r}} \Gamma_{n}(x) \leqslant C_{1} \inf _{B_{r}} \Gamma_{n}(x) \leqslant C_{1} \Gamma_{n}\left(x_{0}\right)=C_{1},
$$

где $C_{1}$ зависит от $\left(N, \lambda_{1}, r\right)$. 
Следовательно, семейство функций $\left\{\Gamma_{n}(x)\right\}$ является ограниченным по $n$ равномерно по $x$ на каждом компакте в $B_{r}$. Применяя диагональньй процесс, выделим подпоследовательность $\left\{\Gamma_{n_{k}}(x)\right\}$, которая сходится к некоторой функции $\Gamma(x)$ равномерно по $x$ на каждом компакте $K$ в $\mathbb{R}^{N}$. Отсюда следует, что $\Gamma_{n_{k}}(x) \underset{n_{k} \rightarrow \infty}{\longrightarrow} \Gamma(x)$ в $L^{2}\left(B_{r}\right)$ при каждом $r>0$. Из оценки (7) тогда получим, что существует подпоследовательность $\Gamma_{n_{k_{i}}}(x)$, которая слабо в $W_{2}^{1, \text { lос }}\left(\mathbb{R}^{N}\right)$ сходится к функции $\Gamma(x)$. Отсюда легко следует, что предельная функция $\Gamma(x)$ является обобшенным решением (из $\left.W_{2}^{1, \text { loc }}\left(\mathbb{R}^{N}\right)\right)$ уравнения $(1)$, удовлетворяюшим условию $\Gamma\left(x_{0}\right)=1$. Ясно также, что $\Gamma(x)>0$ в $\mathbb{R}^{N}$. Остается доказать, что $\Gamma(x)$ обладает свойством

$$
\lim _{|x| \rightarrow \infty} \Gamma(x)=\infty
$$

Вначале докажем, что функция $\Gamma(x)$ является неограниченной в $\mathbb{R}^{N}$. Предположим противное: существует $M>0$ такое, что $0<\Gamma(x) \leqslant M$ в $\mathbb{R}^{N}$.

Применяя для $\Gamma(x)$ построения пп. $1^{\circ}$ и $2^{\circ}$, получим, что сушествует предел $\lim _{|x| \rightarrow \infty} \Gamma(x)=0$. Из (42) тогда легко вытекает, что $\Gamma(x) \equiv 0$. Получили противоречие, ибо $\Gamma\left(x_{0}\right)=1$. Следовательно, $\Gamma(x)$ является неограниченной в $\mathbb{R}^{N}$, т.е. для любого $n \in \mathbb{N}$ сушествует последовательность $x_{n} \in \mathbb{R}^{N},\left|x_{n}\right| \rightarrow \infty(n \rightarrow \infty)$, такая, что

$$
\left.\Gamma(x)\right|_{x=x_{n}}>n
$$

Докажем, что справедливо (45). Предположим противное. Тогда сушествуют $A>$ 0 и последовательность $y_{n} \in \mathbb{R}^{N},\left|y_{n}\right| \rightarrow \infty(n \rightarrow \infty)$, для которых

$$
\left.\Gamma(x)\right|_{x=y_{n}} \leqslant A .
$$

Используя неравенство $(47)$, тот факт, что $Г(x)$ является решением уравнения

$$
L(x) \Gamma+(b(x), \nabla \Gamma)-\frac{\alpha^{2}}{|x|^{2}} \Gamma=0 \quad \text { при }|x|>1
$$

и “послойное” неравенство Харнака [82]

$$
\sup _{\lambda / 2 \leqslant|x| \leqslant 2 \lambda} \Gamma(x) \leqslant C_{1} \inf _{\lambda / 2 \leqslant|x| \leqslant 2 \lambda} \Gamma(x)
$$

с постоянной $C_{1}$, не зависяшей от $\lambda$, легко установить, что имеет место неравенство

$$
\left.\Gamma(x)\right|_{|x|=\left|y_{n}\right|} \leqslant C_{2} A, \quad y_{n} \rightarrow \infty .
$$

Доказательство неравенства (50) является аналогичным доказательству неравенства (26), и мы не будем на нем останавливаться. Отметим только, что при доказательстве неравенства (50) следует применить преобразование подобия $x_{i}=\frac{\lambda_{n}}{4} \sigma_{i}$, где $\lambda_{n}=\left|y_{n}\right|, i=1, \ldots, N$, покрыть сферу $|\sigma|=2$ конечным числом шаров единичного радиуса и использовать неравенства Харнака (44), а затем совершить обратный переход к переменньм $x_{i}$ и воспользоваться неравенством (49). 
Учитывая справедливость неравенства (50), применим к каждому слою

$$
\bar{S}_{\lambda_{n}}=\left\{x \in \mathbb{R}^{N}: \frac{\lambda_{n}}{2} \leqslant|x| \leqslant 2 \lambda_{n}\right\}
$$

неравенство Харнака (49), принцип максимума [82], получим неравенство

$$
\sup _{\left\{x:|x|>\lambda_{n}\right\}} \Gamma(x) \leqslant A C_{2}
$$

Получили противоречие, ибо, как доказано вьше, имеет место неравенство (46). Итак, (45) доказано.

Лемма 11 доказана.

$\mathrm{B} \mathbb{R}^{N}$ рассмотрим уравнение (1), где коэффициент $q(x)$ определен по формуле (36), а коэффициенты $b_{k}(x)(k=1, \ldots, N)$ удовлетворяют условиям $\left(\mathrm{B}_{2}\right)$. Справедливо следующее утверждение.

ЛЕмма 17. І. Для любого $\alpha>0$ существует положительное решение уравнения (1).

II. Для каждого полохсительного решения $\Gamma(x)$ уравнения (1) существует постоянная $l=l(N, \alpha, \lambda)>0$ такая, что

$$
\Gamma(x) \leqslant C_{3}\left(1+|x|^{l}\right) .
$$

ДокАЗАТЕЛЬСТво. І. В каждом шаре $B_{n}(n=1,2, \ldots)$ рассмотрим задачу $(38)$, $(39)$, где $q(x)$ определено равенством (36). Из принципа максимума [82] следует, что $0<V_{n}(x)<M(n=1,2, \ldots)$.

Рассмотрим при некотором $x_{0}$ таком, что $\left|x_{0}\right| \geqslant 2$, семейство функций $(43)$.

Далее, применяя неравенство Харнака (44), оценки Де Джорджи, лемму Арцела и неравенство (7), мы аналогично доказательству леммы 16 в п. $1^{\circ}$ докажем, что сушествует решение $\Gamma(x)$ уравнения $(1)$, удовлетворяющее условиям $\Gamma\left(x_{0}\right)=1$, $\Gamma(x)>0$. Таким образом, утверждение I будем считать доказанным.

Докажем утверждение II. Рассмотрим последовательность колец

$$
\bar{S}_{k}=\left\{x \in \mathbb{R}^{N}: 2^{k-1} \leqslant|x| \leqslant 2^{k}\right\}
$$

Пусть $\alpha>0$ и $\Gamma_{\alpha}(x)$ - одно из отвечаюших ему положительных решений уравнения (1).

Обозначим $M(k)=\sup _{S_{k}} \Gamma_{\alpha}(x)$ и докажем, что имеет место неравенство

$$
M(k+1) \leqslant C M(k), \quad k=1,2, \ldots,
$$

с постоянной $C$, не зависящей от $k$.

Рассмотрим более широкое кольцо

$$
\left\{x \in \mathbb{R}^{N}: 2^{k-2} \leqslant|x| \leqslant 2^{k+1}\right\} .
$$

В этом замкнутом кольце в силу теоремы Вейерштрасса [83] сушествуют точки, в которых $\Gamma_{\alpha}(x)$ достигает $M(k)$ и $M(k+1)$, и так как $\Gamma_{\alpha}(x)$ - решение уравнения $(1)$, то 
для $\Gamma(x)$ имеет место неравенство Харнака вида (49) при $\lambda=2^{n}$ по множеству $S_{k}$. Так как постоянная $C$ в неравенстве (49) не зависит от $\lambda$, то неравенство (52) доказано.

Будем итерировать неравенство (52), спускаясь по индукции от $k$ до $k-1$. Через $k$ шагов из (52) получим неравенство

$$
M(k+1) \leqslant C^{k} M(1) .
$$

Для любого $x \in \mathbb{R}^{N}$ с $|x|>1$ найдется номер $k(k=1,2, \ldots)$, для которого $2^{k-1} \leqslant$ $|x| \leqslant 2^{k}$. Так как $k-1 \leqslant \log _{2}|x| \leqslant k$, то после элементарных преобразований получим неравенство

$$
\begin{aligned}
\Gamma(x) & \leqslant M(k+1) \leqslant M(1) \cdot 2^{k \log _{2} C} \\
& \leqslant M(1) \cdot 2^{\log _{2} C \log _{2}(|x| 2)} \leqslant M(1)(|x| 2)^{\log _{2} C} \leqslant C_{3}(1+|x|)^{l},
\end{aligned}
$$

где $l=\log _{2} C=l\left(N, \alpha, \lambda_{1}\right)>0$.

Лемма 17 доказана.

ЛЕМма 18. В шаре $B_{1}$ рассмотрим задачу

$$
\begin{gathered}
L(x) u+(b(x), \nabla u)+\alpha^{2} q_{1}(x) u=0, \\
\left.u\right|_{|x|=1}=M>0,
\end{gathered}
$$

əде

$$
q_{1}(x) \leqslant q_{0}<0, \quad\left|b_{k}(x)\right|<C, \quad \alpha \geqslant \alpha_{0}>3 .
$$

Существует постоянная $k=k\left(N, \lambda_{1}, q_{0}\right)>0$, для которой

$$
u(0) \leqslant e^{-[\alpha] k} \text {. }
$$

ДокАЗАТЕЛЬСтво. В (55) сделаем замену переменных $x_{i}=y_{i} / \alpha(i=1, \ldots, N)$. Тогда уравнение (55) перейдет в уравнение

$$
\begin{gathered}
L\left(\frac{y}{\alpha}\right) W+\left(b\left(\frac{y}{\alpha}\right), \nabla W\right)+q_{1}\left(\frac{y}{\alpha}\right) W=0, \quad|y|<\alpha \\
\left.W\right|_{|y|=\alpha}=M
\end{gathered}
$$

где $W=u(y / \alpha)$.

В силу условия эллиптичности $(2,1)$ и ограничений $(57)$ получим

$$
\begin{gathered}
\frac{1}{\lambda} \leqslant\left(a\left(\frac{y}{\alpha}\right) \xi, \xi\right) \leqslant \lambda, \quad \frac{1}{\alpha}\left|b_{i}\left(\frac{y}{\alpha}\right)\right| \leqslant \frac{C}{\alpha_{0}} \leqslant C, \quad|\xi|=1, \\
q_{1}\left(\frac{y}{\alpha}\right) \leqslant q_{0}<0 .
\end{gathered}
$$

Из этих неравенств следует, что преобразованное уравнение (59) имеет в шаре $B_{\alpha}$ верхние грани коэффициентов, не зависяшие от $\alpha$. На поверхности сферы $\{|y|=\alpha-1\} \subset B_{\alpha}$ фиксируем любую точку $y_{1}$ такую, что $\left|y_{1}\right|=\alpha-1$. По лемме 15 найдется $\delta(0<\delta<1)$, для которого в центре шара $B_{1}^{y_{1}}$ имеет место неравенство

$$
W\left(y_{1}\right) \leqslant M(1-\delta), \quad\left|y_{1}\right|=\alpha-1 \text {. }
$$


Из произвольности точки $y_{1} \in\{|y|=\alpha-1\}$ следует, что для всех точек сферы $|y|=\alpha-1$ справедливо неравенство

$$
\left.W\right|_{|y|=\alpha-1} \leqslant M(1-\delta) .
$$

Применяя приншип максимума [82], получим, что в замкнутом шаре $\bar{B}_{\alpha-1}$ имеет место неравенство

$$
\left.W\right|_{|y| \leqslant \alpha-1} \leqslant M(1-\delta) .
$$

На поверхности сферы $\{|y|=\alpha-2\} \subset B_{\alpha}$ зафиксируем произвольную точку $y_{2}$, $\left|y_{2}\right|=\alpha-2$, и впишем в $B_{\alpha}$ шар $B_{1}^{y_{2}}$ с центром в $y_{2}$ радиуса 1 . В силу леммы 15 и неравенства (61) получим, что существует постоянная $\delta(0<\delta<1)$, для которой в центре $y_{2}$ шара $B_{1}^{y_{2}},\left|y_{2}\right|=\alpha-2$, имеет место неравенство

$$
W\left(y_{2}\right) \leqslant M(1-\delta)^{2} .
$$

Из произвольности точки $y_{2} \in\{|y|=\alpha-2\}$ и принципа максимума, как и выше, мы легко получим неравенство

$$
\left.W\right|_{|y| \leqslant \alpha-2} \leqslant M(1-\delta)^{2} .
$$

Продолжая аналогичные построения, за $[\alpha]$ шагов мы получим, что решение уравнения (59) удовлетворяет неравенству

$$
\left.W\right|_{|y|=\alpha-[\alpha]} \leqslant M(1-\delta)^{[\alpha]}=M e^{-[\alpha] k}
$$

где $k=\ln \frac{1}{1-\delta}$.

Так как $W(0) \leqslant \sup _{|y|=\alpha-[\alpha]} W(y)$, то, применяя (62) и возврашаясь к переменным $x$, получим, что (58) доказано.

Лемма 18 доказана.

ДокАЗАТЕЛЬСТво ЛЕммы 12. Пусть $\alpha>0$ и $\Gamma_{\alpha}(x)>0-$ решение уравнения (1) с коэффициентом $q(x)(36)$, которое мы построили в лемме 17 . Рассмотрим кольцо $S_{R}^{\prime}=\left\{x \in \mathbb{R}^{N}: R / 4<|x|<R\right\}, R>4$, и сделаем в уравнении (1) преобразование подобия $x_{i}=\frac{R}{4} y_{i}(i=1, \ldots, N)$. При этом уравнение (1) преобразуется в уравнение

$$
L\left(\frac{R}{4} y\right) W+\frac{R}{4}\left(b\left(\frac{R}{4} y\right), \nabla W\right)-\frac{\alpha^{2}}{|y|^{2}} W=0 \quad \text { в } \quad 1<|y|<4,
$$

где $W=\Gamma_{\alpha}\left(\frac{R}{4} y\right)$.

В силу условий равномерной эллиптичности $(2,1)$, условий $\left(\mathrm{B}_{2}\right)$ на $b_{k}(x)(k=1$, $\ldots, N)$ и $(36)$ получим, что верхние грани коэффициентов преобразованного уравнения (63) не зависят от $R$ и что выполнены условия (57) леммы 18 с постоянной $q_{0}=-1 / 4$.

Рассмотрим сферу $\{|y|=2\}$ и впишем в кольцо $\{1 \leqslant|y| \leqslant 4\}$ шар $B_{1}^{y_{1}}$ с центром в любой точке $y_{1} \in\{|y|=2\}$. Из леммы 18 и принципа максимума [82] получим неравенство

$$
W\left(y_{1}\right) \leqslant e^{-k[\alpha]} \sup _{1 \leqslant|y| \leqslant 4} W(y) \leqslant e^{-k[\alpha]} \sup _{1 \leqslant|y| \leqslant 4} W(y) .
$$


Из произвольности точки $y_{1} \in\{|y|=2\}$ и принципа максимума имеем неравенство

$$
\sup _{|y|=2} W(y) \leqslant e^{-k[\alpha]} \sup _{|y|=4} W(y) .
$$

Применяя обозначение $M_{R}=\sup _{|x|=R} \Gamma_{\alpha}(x)$ и возврашаясь к переменным $x$, перепишем последнее неравенство в виде

$$
M_{R / 2} \leqslant e^{-k[\alpha]} M_{R}
$$

Итерируем неравенство (64), заменяя на каждом шаге $R$ на $R / 2$. На втором шаге из (64) следует неравенство

$$
M_{R / 2} \leqslant e^{-k[\alpha]} M_{R / 2} \leqslant e^{-2[\alpha] k} M_{R}
$$

Продолжим процесс итерирования неравенства (64) $n$ раз, где $n$ выберем из условия $1 \leqslant R / 2^{n} \leqslant 2$, т.е. из условия $n \geqslant \log _{2}(R / 2)$, и, учитывая, что $|x| \leqslant R$, и проводя элементарные преобразования, получим неравенство

$$
\begin{aligned}
M_{R} & \geqslant M_{1} e^{k[\alpha] n} \geqslant e^{[\alpha] k \log _{2} \frac{R}{2}} M_{1} \geqslant M_{1} e^{(\alpha-1) k \log _{2} \frac{|x|}{2}} \\
& =2^{\log _{2} e^{k} \cdot(\alpha-1) \log _{2} \frac{|x|}{2}} M_{1}=M_{1}\left(\frac{|x|}{2}\right)^{(\alpha-1) k_{2}}=C_{4}|x|^{(\alpha-1) k_{2}},
\end{aligned}
$$

где

$$
k_{2}=\log _{2} e^{k}, \quad k=\log \frac{1}{1-\delta}, \quad C_{4}=\frac{\sup _{|x| \leqslant 1} \Gamma_{\alpha}(x)}{2^{(\alpha-1) k_{2}}}
$$

Применяя принцип максимума [82], неравенство Харнака (42) и неравенство (65) при $|x|<R$, получим

$$
\Gamma_{\alpha}(x) \geqslant \inf _{|x|=R} \Gamma(x) \geqslant \frac{1}{C_{1}} \sup _{|x|=R} \Gamma(x) \geqslant \frac{C_{3}}{C_{1}}|x|^{(\alpha-1) k_{2}}=C_{5}|x|^{(\alpha-1) k_{2}} .
$$

Используя (66), завершим доказательство леммы 12. По данному $m>0$ найдем число $\alpha_{0}$ из равенства $k_{2}(\alpha-1)=m$, т.е. $\alpha_{0}=1+m / k_{2}$, и, согласно лемме 17 , найдем решение $\Gamma_{\alpha_{0}}(x)$ уравнения (1), для которого (в силу (66)) будет справедливо неравенство

$$
\Gamma_{\alpha_{0}}(x)>C\left(|x|^{m}+1\right)
$$

Лемма 12 доказана.

СлЕДСТВИЕ. Для каждого $\alpha_{0}>0$ и каждого решения уравнения (1) $\Gamma_{\alpha_{0}}(x)$ при $\alpha_{1}>\alpha_{0}$ найдется решение $\Gamma_{\alpha_{1}}(x)$ уравнения (1), для которого имеет место неравенство

$$
\Gamma_{\alpha_{1}}(x)>\Gamma_{\alpha_{0}}(x), \quad|x| \geqslant a,
$$

əде $a=a\left(\alpha_{0}, \alpha_{1}, N, \lambda\right)$. 
ДокАЗАТЕЛЬСТВо. Из оценки (51) леммы 17 для каждого $\alpha_{0}$ и каждого решения $\Gamma_{\alpha_{0}}$ уравнения (1) получаем

$$
\Gamma_{\alpha_{0}}(x) \leqslant C_{3}(1+|x|)^{l}, \quad l=l\left(N, \alpha_{0}, \lambda_{1}\right) .
$$

Для фиксированного $\alpha_{0}$ выберем $\alpha_{1}$ из неравенства

$$
\left(\alpha_{1}-1\right) k_{2} \geqslant l\left(N, \alpha_{0}, \lambda_{1}\right)
$$

и тогда по лемме 12 найдем решение $\Gamma_{\alpha_{1}}(x)$ уравнения $(1)$, удовлетворяюшее в силу (65) неравенству

$$
\Gamma_{\alpha_{1}}(x) \geqslant C_{5}|x|^{\left(\alpha_{1}-1\right) k_{2}} .
$$

Из неравенств (68), (69) получим требуемое утверждение:

$$
\begin{aligned}
& \Gamma_{\alpha_{1}}(x)-\Gamma_{\alpha_{0}}(x) \geqslant C_{5}|x|^{\left(\alpha_{1}-1\right) k_{2}}-C_{3}|x|^{l\left(N, \alpha_{0}, \lambda\right)} \\
& \quad=|x|^{\left(\alpha_{1}-1\right) k_{2}}\left(C_{5}-C_{3}|x|^{l-k_{2}\left(\alpha_{1}-1\right)}\right)>0 \text { при }|x| \geqslant a=\left(\frac{C_{5}}{C_{3}}\right)^{\frac{1}{\left(\alpha_{1}-1\right) k_{2}-l}} .
\end{aligned}
$$

Следствие доказано.

При доказательстве теоремы 3 нам потребуется следующее утверждение.

ЛЕмма 19. Пусть коэффициенты $b_{k}(x)(k=1, \ldots, N)$ удовлетворяют условиям $\left(\mathrm{B}_{2}\right)$, коэффициент $q(x)$ определен равенством

$$
q(x)= \begin{cases}-\frac{\alpha^{2}}{|x|^{2}(\ln |x|)^{s}} & \text { npu }|x|>2,0<s \leqslant 1, \alpha>0 \\ 0 & \text { npu }|x| \leqslant 2 .\end{cases}
$$

Тогда существует функция $\Gamma(|x|)=\Gamma(x)$ такая, что:

1) $\Gamma(x)>0$; 2) $\Gamma(x) \in W_{2}^{1, \operatorname{loc}_{(\mathbb{R}}}\left(\mathbb{R}^{N}\right)$; 3$) \lim _{|x| \rightarrow \infty} \Gamma(|x|)=\infty$;

4) $\Gamma(x)$ является обобщенным решением неравенства

$$
\Delta \Gamma+(b(x), \nabla \Gamma)+q(x) \Gamma \leqslant 0 \quad \text { в } \quad \mathbb{R}^{N},
$$

т.е. имеет место интегральное неравенство

$$
\int_{\mathbb{R}^{N}}[-(\nabla \Gamma, \nabla \varphi)+((b, \nabla \Gamma)+q \Gamma) \varphi] \mathrm{d} x \leqslant 0
$$

для любой $\varphi(x) \geqslant 0$ из класса $C_{0}^{1}\left(\mathbb{R}^{N}\right)$. 
ДокАЗАТЕльство. Будем искать решение неравенств $(71),(72)$ в виде $\Gamma=\Gamma(r)$, $r=|x|$, где $\Gamma(r)>0, \Gamma_{r}(r) \geqslant 0$ при $r>0$. Учитьвая условия $\left(\mathrm{B}_{2}\right)$ и формулу $(70)$, будем иметь

$$
\begin{aligned}
\Delta \Gamma+(b, \nabla \Gamma)+q \Gamma & =\Gamma_{r r}+\frac{N-1}{r} \Gamma_{r}+\sum_{k=1}^{N} b_{k} \frac{x_{k}}{r} \Gamma_{r}+q \Gamma \\
& \leqslant \Gamma_{r r}+\frac{N-1+B}{r} \Gamma_{r}+q(r) \Gamma \text { при } r>2 .
\end{aligned}
$$

Пусть $\Gamma(r)$ - решение следуюшего уравнения:

$$
\Gamma_{r r}+\frac{N-1+B}{r} \Gamma_{r}-\frac{\alpha^{2}}{r^{2}(\ln r)^{s}} \Gamma=0, \quad r>2,
$$

удовлетворяющее условиям

$$
\left.\Gamma\right|_{r=2}=1,\left.\quad \Gamma_{r}\right|_{r=2}=0
$$

Тогда правая часть (73) равна нулю. В уравнении (74) проведем замену $t=\ln r$. Тогда $\Gamma_{r}=\frac{1}{r} \Gamma_{t}, \Gamma_{r r}=\frac{1}{r^{2}}\left(\Gamma_{t t}-\Gamma_{t}\right)$, и равенства $(74),(75)$ приобретают вид

$$
\begin{gathered}
\Gamma_{t t}+(N-2+B) \Gamma_{t}-\frac{\alpha^{2}}{t^{s}} \Gamma=0, \quad t>t_{0}=\ln 2 \\
\left.\Gamma\right|_{t=t_{0}}=1,\left.\quad \Gamma_{t}\right|_{t=t_{0}}=0
\end{gathered}
$$

Докажем, что $\Gamma(t)>1$ при $t>t_{0}$. Предположим противное, т.е. что существует $t_{1}>t_{0}$, для которого $\Gamma\left(t_{1}\right) \leqslant 1$. Так как $\Gamma\left(t_{0}\right)=1$ и $\Gamma\left(t_{1}\right)<1$, то сушествует точка $t_{2} \in\left(t_{0}, t_{1}\right)$, в которой $\Gamma(t)$ достигает максимума, т.е.

$$
\Gamma\left(t_{2}\right)>0, \quad \Gamma_{t}\left(t_{2}\right)=0, \quad \Gamma_{t t}\left(t_{2}\right) \leqslant 0 .
$$

Тогда уравнение $(75)$ в $t=t_{2}$ преврашается в равенство

$$
\Gamma_{t t}\left(t_{2}\right)=\frac{\alpha^{2}}{t_{2}^{s}} \Gamma\left(t_{2}\right)
$$

правая часть которого положительна, а левая часть не положительна.

Полученное противоречие доказьвает, что $\Gamma(t)>1$ при $t>t_{0}$. Умножим (76) на $e^{\gamma t}$, где $\gamma=N+B-2$, и проинтегрируем по $t$ от $t_{0}$ до $t$. Будем иметь

$$
\int_{t_{0}}^{t}\left(e^{\gamma \tau} \Gamma_{\tau}\right)_{\tau} \mathrm{d} \tau=\alpha^{2} \int_{t_{0}}^{t} \Gamma e^{\gamma \tau} \frac{\mathrm{d} \tau}{\tau^{s}}
$$

T.e.

$$
e^{\gamma t} \Gamma_{t}(t)-e^{\gamma t_{0}} \Gamma_{t}\left(t_{0}\right)>\frac{\alpha^{2}}{t^{s}} \int_{t_{0}}^{t} e^{\gamma \tau} \mathrm{d} \tau=\frac{\alpha^{2}}{t^{s} \gamma}\left(e^{\gamma t}-e^{\gamma t_{0}}\right)
$$


Учитывая, что $\Gamma_{t}\left(t_{0}\right)=0$ и поделив на $e^{\gamma t}$, получим

$$
\Gamma_{t} \geqslant \frac{\alpha^{2}}{\gamma t^{s}}\left(1-e^{-\gamma\left(t-t_{0}\right)}\right)
$$

Выберем $T=T\left(\gamma, \alpha, t_{0}\right)$ так, чтобы $1-e^{-\gamma\left(t-t_{0}\right)}>1 / 2$ при $t>T>t_{0}$. Достаточно положить

$$
T=\frac{\ln 2+t_{0} \gamma}{2}
$$

тогда при $t \geqslant T$ из последнего неравенства получим

$$
\Gamma_{t} \geqslant \frac{\alpha^{2}}{2 \gamma t^{s}}, \quad 0<s \leqslant 1
$$

Интегрируя это неравенство по $t$ при $s \in(0,1)$, получим

$$
\Gamma(t)-\Gamma(T)>\frac{\alpha^{2}}{2 \gamma(1-s)}\left[t^{1-s}-T^{1-s}\right], \quad t>T
$$

Так как $0<s<1$, то $\Gamma(t)>C_{1} t^{1-s}$ при достаточно большом $T_{1} \geqslant T$. Возврашаясь к переменной $r, r=e^{t}$, получим

$$
\Gamma(r) \geqslant C_{1}(\ln r)^{1-s}, \quad r \geqslant e^{T}, \quad 0<s<1
$$

Из (79) следует, что $\lim _{r \rightarrow \infty} \Gamma(r)=+\infty$.

При $s=1$ интегрируем (78) по $t$, тогда

$$
\Gamma(t)-\Gamma(T)>\frac{\alpha^{2}}{2 \gamma}(\ln t-\ln T) \rightarrow \infty, \quad t \rightarrow \infty
$$

Из (79) и (80) следует, что функци

$$
\Gamma= \begin{cases}1 & \text { при } r \leqslant 2, \\ \Gamma(r) & \text { при } r \geqslant 2,\end{cases}
$$

где $\Gamma(r)$ - решение задачи $(74),(75)$, удовлетворяет требуемым свойствам 1)-4) леммы 19.

Лемма 19 доказана.

Для доказательства теоремы 5 нам потребуется следующее утверждение.

ЛЕмма 20. Пусть коэффициенты $b_{k}(x)(k=1, \ldots, N)$ удовлетворяют условиям $\left(\mathrm{B}_{2}\right)$ и коэффициент $q(x)$ имеет вид (36). Тогда существует функция $\Gamma(r)>0$, для которой выполнены условия:

1) $\Gamma(r)>0$; 2) $\Gamma(r) \in W_{2}^{1,{ }^{l o c}}\left(\mathbb{R}^{N}\right)$; 3$) \lim _{r \rightarrow \infty} \Gamma(r)=\infty$;

$\Gamma(r)$ удовлетворяет интегральному неравенству (72) при $q(x)$, определенному $\boldsymbol{\theta}(36)$. 
ДокАЗАТЕЛЬСтво. Будем искать решение неравенства $(71)$ или $(72)$ в виде $\Gamma=$ $\Gamma(r)$, где $\Gamma(r)>0, \Gamma^{\prime}(r) \geqslant 0$ при $r>0$. Учитывая условия $\left(\mathrm{B}_{2}\right)$ и формулу $(36,1)$, будем иметь

$$
\begin{aligned}
\Delta \Gamma+(b(x), \nabla \Gamma)+q(x) \Gamma & \leqslant \Gamma_{r r}+\frac{N-1}{r} \Gamma_{r}+\sum_{k=1}^{N} b_{k}(x) \frac{x_{k}}{r} \Gamma_{r}-\frac{\alpha^{2}}{r^{2}} \Gamma \\
& \leqslant \Gamma_{r r}+\frac{N-1+B}{r} \Gamma_{r}-\frac{\alpha^{2}}{r^{2}} \Gamma .
\end{aligned}
$$

Пусть $\Gamma(r)>0$ - решение уравнения

$$
\Gamma_{r r}+\frac{N-1+B}{r} \Gamma_{r}-\frac{\alpha^{2}}{r^{2}} \Gamma=0, \quad r>1,
$$

удовлетворяющее условиям

$$
\left.\Gamma\right|_{r=1}=1,\left.\quad \Gamma_{r}\right|_{r=1}=0,
$$

тогда функция $\Gamma(|x|)$ удовлетворяет в силу (82) неравенству

$$
\Delta \Gamma+(b(x), \nabla \Gamma)+q(x) \Gamma \leqslant 0 \text { при }|x|>1 .
$$

Будем искать решение уравнения (83) в виде $\Gamma=r^{l}$. Вставляя $\Gamma=r^{l}$ в (83), получим уравнение

$$
l^{2}+(N-2+B) l-\alpha^{2}=0,
$$

которое имеет корни

$$
l_{1}=\frac{-Q+\sqrt{Q^{2}+4 \alpha^{2}}}{2}>0, \quad l_{2}=\frac{-Q-\sqrt{Q^{2}+4 \alpha^{2}}}{2},
$$

где

$$
Q=N+B-2 .
$$

Решение задачи (83), (84) имеет вид

$$
\Gamma(r)=C_{1} r^{l_{1}}+C_{2} r^{l_{2}},
$$

где

$$
C_{1}=\frac{l_{2}}{l_{1}-l_{2}}>0, \quad C_{2}=\frac{l_{1}}{l_{1}-l_{2}} .
$$

Определим теперь обобщенное решение $\Gamma_{1}(x)$ неравенства $(82)$ в $\mathbb{R}^{N}$, полагая

$$
\Gamma_{1}(x)= \begin{cases}\Gamma(|x|) & \text { при }|x|>1, \\ 1 & \text { при }|x| \leqslant 1,\end{cases}
$$

где $\Gamma(|x|)$ - решение задачи (83), (84), определенное формулой (87).

Функция (88) по построению принадлежит $W_{2}^{1, \text { loc }}\left(\mathbb{R}^{N}\right)$ и, кроме того, очевидно удовлетворяет неравенству (82) и, следовательно, и в интегральном смысле (72).

Очевидно также, что функция (88) обладает следуюшими свойствами:

1) $\left.\left.\Gamma_{1}(x)>0 ; \quad 2\right) \Gamma_{1}(x) \in W_{2}^{1, \text { loc }}\left(\mathbb{R}^{N}\right) ; \quad 3\right) \lim _{|x| \rightarrow \infty} \Gamma_{1}(|x|)|x|^{-l_{1}}=C_{1}>0$.

Так как в силу (86) $l_{1}>0$, то $\Gamma_{1}(x) \sim C_{1}|x|^{l_{1}} \rightarrow \infty$ при $|x| \rightarrow \infty$. Следовательно, лемма 20 доказана. 


\section{§. Доказательство результатов п. $4^{\circ} \S 1$}

В бесконечном по $t$ цилиндре $\{x, t:|x|<R, t>0\}$ рассмотрим уравнение

$$
L(x) u+(b(x), \nabla u)+c(x) u-u_{t}=0,
$$

где $a_{i k}(x)=a_{k i}(x)$, выполнено условие $(2,1)$ параболичности, коэффициенты уравнения (1) измеримы и ограничены при $|x|<R$ и $c(x) \leqslant 0$ при $|x|<R$.

Пусть $p(x, t)$ - решение уравнения (1), удовлетворяющее условиям

$$
\left.p\right|_{|x|=R}=0, \quad t>0, \quad p(x, 0)=\varphi(x),
$$

где $\varphi(x) \in C\left(B_{R}\right)$.

Напомним [39], что $p(x, t)$ назьвается обобщенным решением задачи (1), (2), если $V(x, t)$ принадлежит классу $W_{2}^{1,0}\left(B_{R} \times(0, T)\right), T>0$, удовлетворяет первому из равенств (2) и справедливо интегральное тождество

$\int_{0}^{T} \int_{|x| \leqslant R}\left[-\eta_{t} p+(a(x) \nabla p, \nabla \eta)-\eta((b(x), p)+c(x) p)\right] \mathrm{d} x \mathrm{~d} t=\int_{|x| \leqslant R} \varphi(x) \eta(x, 0) \mathrm{d} x$ для всех функций $\eta(x, t)$ из $W_{2}^{1,1}\left(B_{R} \times(0, T)\right)$, удовлетворяюших условиям

$$
\left.\eta\right|_{|x|<R, t=T}=0,\left.\quad \eta\right|_{|x|=R, 0<t<T}=0 .
$$
[46].

Сушествование и единственность решения задачи (1), (2) хорошо известны [5], [39],

Лемма 21. Решение задачи (1), (2) имеет предел

$$
\lim _{t \rightarrow \infty} p(x, t)=0
$$

равномерно по $x \in B_{R}$.

ЗАмЕчАниЕ. Если коэффициенты уравнения - гладкие функции, то утверждение, аналогичное лемме 21 , хорошо известно (см. теорему 2 из [5; 12$]$ ). Используя известньй результат о существовании единственной положительной собственной функции $p(x)$, отвечающей положительному собственному значению $\lambda_{0}$ первой краевой задачи

$$
L(x) p+(b(x), \nabla p)+c(x) p+\lambda p=0,\left.\quad p\right|_{|x|=2 R}=0,
$$

установленный в работах [87], [88], с помошью теоремы М.Г. Крейна, М. А. Рутмана [89], можно легко доказать лемму 21. Ради краткости не будем этого делать.

ДОКАЗАТЕЛЬСТВО ТЕОРЕМ 1-3 бУдем Проводить одновременно, ибо они оПираются только на свойства 1$)-3$ ) функций $\Gamma(x)$, приведенные в леммах 10,11 и 19 и обоснованные в $\S 2$.

Итак, пусть $\Gamma(x)>0$ - функция из класса $W_{2}^{1, \text { loc }}\left(\mathbb{R}^{N}\right)$ такая, что

$$
\lim _{|x| \rightarrow \infty} \Gamma(x)=+\infty
$$


и $Г(x)$ является обобщенным решением $($ см. $(2,1))$ из $W_{2}^{1, \text { loc }}\left(\mathbb{R}^{N}\right)$ уравнения (неравенства)

$$
L(x) \Gamma+(b(x), \nabla \Gamma)+q(x) \Gamma=0(\leqslant 0), \quad x \in \mathbb{R}^{N},
$$

где при $N=1$ или $N=2$ коэффициенты $b_{k}(x)$ удовлетворяют условиям $\left(\mathrm{B}_{1}\right)$, а коэффициент $q(x)$ определен равенством $(2,2)$. При $N \geqslant 3$ коэффициенты $b_{k}(x)$ $(k=1, \ldots, N)$ удовлетворяют условиям $\left(\mathrm{B}_{2}\right)$, а коэффициент $q(x)$ определен равенством $(36,2)$ в случае теоремы 2 и равенством $(70,2)$ в случае теоремы 3.

Наряду с задачей Коши $(77,1),(78,1)$ мы рассмотрим задачу Коши

$$
\begin{gathered}
L(x) W+(b(x), \nabla W)+q(x) W-W_{t}=0, \quad(x, t) \in \mathbb{R}_{+}^{N+1}, \\
W(x, 0)=\left|u_{0}(x)\right|, \quad x \in \mathbb{R}^{N}
\end{gathered}
$$

где $q(x)$ определен формулой $(2,2)$ при $N=1$ или $N=2$, а при $N \geqslant 3 q(x)$ определен по формуле $(36,2)$ или по формуле $(70,2)$.

Из принципа максимума [12], [39] легко получаем следующее утверждение.

Лемма 22. Для решений задач Коши $(77,1),(78,1)$ и (6), (7) справедливо неравенство

$$
|u(x, t)| \leqslant W(x, t), \quad(x, t) \in \mathbb{R}_{+}^{N+1} .
$$

Из (8) следует, что для доказательства теорем 1-3 из п. $4^{\circ} \S 1$ достаточно доказать, что существует

$$
\lim _{t \rightarrow \infty} W(x, t)=0
$$

равномерно по $x \in K$ в $\mathbb{R}^{N}$.

Фиксируем компакт $K \subset \mathbb{R}^{N}$ и выберем $r>0$ так, чтобы $\bar{B}_{r} \supset K$. Для любого $\varepsilon>0$ выберем $\delta=\delta(N, r)>0$ такое, что

$$
\delta \Gamma(x)<\frac{\varepsilon}{2}, \quad x \in \bar{B}_{r}
$$

где $\Gamma(x)$ - положительное решение соответствуюшего эллиптического уравнения (5), для которого справедливо (4) (леммы 10, 11, 19).

Рассмотрим функцию

$$
V(x, t)=\delta \Gamma(x)-W(x, t),
$$

где Г $(x)$ - функция из лемм $10,11,19$, а $W(x, t)$ - решение задачи $(6),(7)$. Так как $W(x, t)$ является ограниченной функцией, $0<W(x, t) \leqslant M$, в $\mathbb{R}_{+}^{N+1}$, а функция $\Gamma(x)$ удовлетворяет предельному равенству (4), то сушествует $R, R>r$, такое, что

$$
\left.V(x, t)\right|_{|x|=R} \geqslant 0 \text { для всех } t>0 .
$$

Ясно также, что функция $V(x, t)(11)$ удовлетворяет уравнению (неравенству)

$$
L(x) V+(b(x), \nabla V)+q(x) V-V_{t}=0(\leqslant 0), \quad|x|<R, \quad t>0,
$$

и условию

$$
V(x, 0)=\delta \Gamma(x)-\left|u_{0}(x)\right|, \quad x \in B_{R} .
$$


Введем обозначение

$$
\varphi(x) \equiv \delta \Gamma(x)-\left|u_{0}(x)\right|, \quad|x|<R .
$$

Рассмотрим задачу

$$
\begin{gathered}
L(x) p+(b(x), \nabla p)+q(x) p-p_{t}=0(\leqslant 0), \quad|x|<R, \quad t>0, \\
\left.p\right|_{|x|=R}=0, \\
p(x, 0)=\varphi^{-}(x), \quad|x|<R,
\end{gathered}
$$

где $\varphi^{-}(x)=\min _{|x|<R}(\varphi(x), 0) \leqslant 0$.

Из принципа максимума [12], [39] тогда следует, что $p(x, t) \leqslant 0$ при $|x|<R, t>0$. Из леммы 21 вытекает, что сушествует предел (3) равномерно по $x \in B_{R}$, т.е. для любого $\varepsilon>0$ сушествует $T(\varepsilon)>0$ такое, что при $t \geqslant T(\varepsilon)$ справедливо

$$
p(x, t)>-\frac{\varepsilon}{2}, \quad x \in B_{R} .
$$

Введем функцию

$$
r(x, t)=V(x, t)-p(x, t),
$$

где $V(x, t)$ - решение уравнения (13), удовлетворяющее условиям (12) и $(14)$, a $p(x, t)-$ решение задачи (16)-(18).

Ясно, что функция $r(x, t)$ удовлетворяет уравнению (неравенству) (16) при $|x|<R$, $t>0$ и условиям

$$
\left.r(x, t)\right|_{|x|=R} \geqslant 0, \quad t>0, \quad r(x, 0)=\varphi(x)-\varphi^{-}(x)=\varphi^{+}(x) \geqslant 0,
$$

где $\varphi^{+}(x)=\max _{|x|<R}(\varphi(x), 0) \geqslant 0$. Следовательно, по принципу максимума [39]

$$
r(x, t) \geqslant 0, \quad|x|<R, \quad t>0 .
$$

Учтем далее, что при $|x|<r$ имеет место неравенство (10), а при $t \geqslant T(\varepsilon)$ справедливо неравенство (19), тогда из (20), (10) и (19) получим

$$
0 \leqslant W(x, t) \leqslant \delta \Gamma(x)-p(x, t)<\varepsilon \text { при } t>T(\varepsilon)
$$

равномерно по $x \in \bar{B}_{r}$, а поэтому в силу (8) получим, что сушествует предел

$$
\lim _{t \rightarrow \infty} u(x, t)=0
$$

равномерно на $K$ в $\mathbb{R}^{N}$.

Теоремы $1-3$ из п. $4^{\circ} \S 1$ доказаны.

Рассмотрим две задачи Коши, задачу $(77,1),(78,1)$ и задачу

$$
\begin{gathered}
L(x) V_{\alpha}+\left(b(x), \nabla V_{\alpha}\right)+q_{\alpha}(x) V_{\alpha}-V_{t}=0, \quad(x, t) \in \mathbb{R}^{N}, \\
V_{\alpha}(x, 0)=\left|u_{0}(x)\right|, \quad x \in \mathbb{R}^{N},
\end{gathered}
$$

где функция $u_{0}(x)$, которая определена равенством $(78,1)$, и функция $(24)$ удовлетворяют условию роста $(81,1)$, коэффициент $q_{\alpha}(x)$ в $(23)$ определен равенством $(36,2)$, а коэффициенты $b_{i}(x)$ удовлетворяют условию $\left(\mathrm{B}_{2}\right)$. 
ЛЕмма 23. При сформулированных условиях имеет место неравенство

$$
|u(x, t)| \leqslant V_{\alpha}(x, t) .
$$

Доказательство леммы является прямым следствием принципа максимума [39], [12] и может быть опушено.

Пусть $V_{\alpha_{1}}(x, t)$ и $V_{\alpha_{2}}(x, t)$ - решения задачи Коши $(23),(24)$ с одними и теми же коэффициентами $a_{i k}(x), b_{i}(x)$, начальной функцией $\left|u_{0}(x)\right|$, но с различньми коэффишиентами $q_{\alpha_{1}}(x), q_{\alpha_{2}}(x)$, определенными в $(36,2)$ при $\alpha=\alpha_{1}$ и $\alpha=\alpha_{2}$ соответственно.

ЛЕмма 24. Если $\alpha_{1} \geqslant \alpha_{2}$, то имеет место неравенство

$$
V_{\alpha_{1}}(x, t) \leqslant V_{\alpha_{2}}(x, t) \quad \text { в } \quad \mathbb{R}_{+}^{N+1} .
$$

ДокАЗАТЕльство. Рассмотрим функцию

$$
z(x, t)=V_{\alpha_{2}}(x, t)-V_{\alpha_{1}}(x, t) .
$$

Тогда для $z(x, t)$ получим задачу

$$
\begin{gathered}
L(x) z+(b, \nabla z)+q_{\alpha_{2}} z-z_{t}=-V_{\alpha_{1}}\left(q_{\alpha_{2}}-q_{\alpha_{1}}\right) \quad \text { в } \mathbb{R}_{+}^{N+1}, \\
z(x, 0)=0, \quad x \in \mathbb{R}^{N}
\end{gathered}
$$

решение которой имеет вид

$$
z(x, t)=\int_{0}^{t} \mathrm{~d} \tau \int_{\mathbb{R}^{N}} G_{\alpha_{2}}(x, y ; t, \tau) V_{\alpha_{1}}(y, \tau)\left(q_{\alpha_{2}}(y)-q_{\alpha_{1}}(y)\right) \mathrm{d} y,
$$

где $G_{\alpha_{2}}(x, y ; t, \tau)>0$ - фундаментальное решение уравнения (23) при $\alpha=\alpha_{2}$ (см. [90]). Так как $q_{\alpha_{1}}(y) \leqslant q_{\alpha_{2}}(y)$ при $\alpha_{2} \leqslant \alpha_{1}$ и $V_{\alpha_{1}} \geqslant 0$, то $z(x, t)>0$, т.е. $(26)$ доказано.

Пусть вьполнено условие роста $(81,1)$; применяя лемму 12 , найдем $\alpha_{0}=\alpha_{0}(m$, $\left.N, \lambda_{1}\right)>0$ и решение $\Gamma_{\alpha_{0}}(x)$ уравнения $(1,2)$ (где коэффищиент $q(x)$ определен равенством $(36,2)$ при $\alpha=\alpha_{0}$, а коэффициенты $b_{i}(x)$ удовлетворяют условию $\left.\left(\mathrm{B}_{2}\right)\right)$, для которого имеет место неравенство

$$
\Gamma_{\alpha_{0}}(x) \geqslant C|x|^{m}, \quad|x|>1
$$

Фиксируем эти $m>0$ и $\alpha_{0}$.

Лемма 25. Для выбранных $m>0$ и $\alpha_{0}$ и каждого $m_{1}>m$ найдутся постоянная $\alpha_{1}=\alpha_{1}\left(m_{1}, N, \lambda_{1}\right)>\alpha_{0}$ и решение $\Gamma_{\alpha_{1}}$ уравнения $(1,2)$ такие, что виполнено неравенство

$$
\Gamma_{\alpha_{1}}(x) \geqslant C|x|^{m_{1}}, \quad x \in \mathbb{R}^{N},
$$

и для решения задачи Коши (23), (24) при $\alpha=\alpha_{1}$ справедливо неравенство

$$
V_{\alpha_{1}}(x, t) \leqslant C \Gamma_{\alpha_{1}}(x), \quad x \in \mathbb{R}^{N}
$$


ДоКАЗАТЕЛЬСТвО. Пусть $m_{1}>m$, тогда в силу следствия леммы 12 (приведенного в $\S 2$ сразу после доказательства леммы 12$)$ найдутся $\alpha_{1}>\alpha_{0}$ и решение $\Gamma_{\alpha_{1}}(x)$ уравнения $(1,2)$, для которых справедливо (29). Рассмотрим функцию

$$
z(x, t)=C_{5} \Gamma_{\alpha_{1}}(x)-V_{\alpha_{1}}(x, t),
$$

где $C_{5}>0$ будет выбрано ниже, а функция $V_{\alpha_{1}}(x, t)$ - решение задачи Коши $(23),(24)$. Функция $z(x, t)$ удовлетворяет тому же уравнению $(23)$ и начальному условию $z(x, 0)=C \Gamma_{\alpha_{1}}(x)-\left|u_{0}(x)\right|$. Так как $\left|u_{0}(x)\right|$ удовлетворяет условию роста $(81,1)$ и для $\Gamma_{\alpha_{1}}(x)$ справедливо $(29)$ с $m_{1}>m$, то сушествует $C>0$, для которого $z(x, 0) \geqslant 0$, $x \in \mathbb{R}^{N}$. Тогда из принщипа максимума [12], [39] получим, что $z(x, t) \geqslant 0$ внутри слоя $H=\left\{x, t: x \in \mathbb{R}^{N}, 0<t \leqslant T\right\}$ для любого $T>0$. Из произвольности $T>0$ следует, что неравенство (29) обосновано.

Лемма 25 доказана.

В силу леммы 23 для доказательства теоремы 4 достаточно доказать следующее утверждение.

ТЕОРема 6. Пусть для $\left|u_{0}(x)\right|$ выполнено условие $(81,1)$, коэффициенты $b_{k}(x)$ $(k=1, \ldots, N)$ удовлетворяют условиям $\left(\mathrm{B}_{2}\right)$, тогда существует постоянна $\alpha_{2}=\alpha_{2}\left(m, N, \lambda_{1}\right)>0$ такая, что решение задачи Коши $(23),(24) V_{\alpha_{2}}(x, t)$ имеет предел

$$
\lim _{t \rightarrow \infty} V_{\alpha_{2}}(x, t)=0
$$

равномерно по $x$ на каждом компакте $K \subset \mathbb{R}^{N}$.

ДокАЗАТЕЛЬСТВо. Зафиксируем компакт $K$ в $\mathbb{R}^{N}$ и выберем $r>0$ так, чтобы $\bar{B}_{r} \supset K$.

Пусть $m_{1}>m$, тогда в силу леммы 12 найдем $\alpha_{1}>0$ такое, чтобы было вьполнено (29). Для выбранного $\alpha_{1}$ в силу следстви леммы 12 найдем $\alpha_{2}>\alpha_{1}$ и найдем решение $\Gamma_{\alpha_{2}}(x)$ уравнения $(1,2)$, удовлетворяюшее неравенствам

$$
\begin{gathered}
\Gamma_{\alpha_{2}}(x)>\Gamma_{\alpha_{1}}(x) \text { при }|x| \geqslant a, \\
\Gamma_{\alpha_{2}}(x) \geqslant C\left(1+|x|^{m_{1}}\right) .
\end{gathered}
$$

Для любого $\varepsilon>0$ выберем $\delta>0$, для которого

$$
\delta \Gamma_{\alpha_{2}}(x)<\frac{\varepsilon}{2}, \quad x \in \bar{B}_{r} .
$$

Рассмотрим функцию

$$
W(x, t)=\delta \Gamma_{\alpha_{2}}(x)-V_{\alpha_{2}}(x, t),
$$

где $V_{\alpha_{2}}(x, t)$ - решение задачи Коши $(23),(24)$.

Так как $\alpha_{2}>\alpha_{1}$, то в силу (26) и (30) получим

$$
V_{\alpha_{2}}(x, t) \leqslant V_{\alpha_{1}}(x, t) \leqslant C \Gamma_{\alpha_{1}}(x), \quad t>0, \quad x \in \mathbb{R}^{N} .
$$


Из оценок $(65,2)$ и $(51,2)$, установленных в доказательстве леммы 12 и лемме 17 соответственно, получим

$$
\begin{aligned}
W(x, t) & \geqslant \delta \Gamma_{\alpha_{2}}(x)-V_{\alpha_{1}}(x, t) \geqslant \delta \Gamma_{\alpha_{2}}(x)-C \Gamma_{\alpha_{1}}(x) \geqslant \delta C_{5}|x|^{\left(\alpha_{2}-1\right) k_{2}}-C_{3}|x|^{l_{1}} \\
& =|x|^{\left(\alpha_{2}-1\right) k_{2}}\left(\delta C_{5} C_{3}|x|^{l\left(\alpha_{1}\right)-\left(\alpha_{2}-1\right) k}\right)>0
\end{aligned}
$$

при

$$
|x| \geqslant a_{1}=\left(\frac{\delta C_{5}}{C_{3}}\right)^{\frac{1}{\left(\alpha_{2}-1\right) k_{2}-l\left(\alpha_{1}\right)}}>0
$$

если $\alpha_{2}$ выбрать из условия $\left(\alpha_{2}-1\right) k_{2}>l\left(\alpha_{1}\right)$.

В силу $(36)$ сушествует $R \geqslant \max \left(r, a_{1}\right)$ такое, что справедливо неравенство

$$
\left.W(x, t)\right|_{|x|=R} \geqslant 0 \text { для } t>0 .
$$

Поэтому функшия (35) удовлетворяет соотношениям

$$
\begin{gathered}
L(x) W+(b(x), \nabla W)+q_{\alpha_{2}}(x) W-W_{t}=0, \quad(x, t) \in \mathbb{R}_{+}^{N+1}, \\
\left.W\right|_{|x|=R} \geqslant 0, \quad t>0, \\
\left.W\right|_{t=0}=\delta \Gamma_{\alpha_{2}}(x)-C\left(1+|x|^{m}\right) .
\end{gathered}
$$

Рассмотрим функции $z(x, t)=W(x, t)-p(x, t)$, где $W(x, t)-$ функция $(35)$, а $p(x, t)$ является решением задачи

$$
\begin{gathered}
L(x) p+(b(x), \nabla p)+q_{\alpha_{2}}(x) p-p_{t}=0, \quad|x|<R, \quad t>0, \\
\left.p\right|_{|x|=R}=0, \quad t>0, \\
p(x, 0)=\min \left(0, \delta \Gamma_{\alpha_{2}}(x)-C\left(1+|x|^{m}\right)\right), \quad|x|<R .
\end{gathered}
$$

В лемме 21 установлено, что существует предел (3), т.е. для любого $\varepsilon>0$ имеет место неравенство

$$
p(x, t)>-\frac{\varepsilon}{2} \text { при } t>T(\varepsilon), \quad x \in B_{R} .
$$

Функция $z(x, t)$ удовлетворяет уравнению

$$
L(x) z+(b(x), \nabla z)+q_{\alpha_{2}}(x) z-z_{t}=0, \quad|x|<R, \quad t>0,
$$

и условиям

$$
\begin{gathered}
\left.z(x, t)\right|_{|x|=R} \geqslant 0, \quad t>0 \\
z(x, 0)=\max \left(0, \delta \Gamma_{\alpha_{2}}(x)-C\left(1+|x|^{m}\right)\right) \geqslant 0 .
\end{gathered}
$$

Следовательно, по принципу максимума [12], [39] получим $z(x, t) \geqslant 0,|x| \leqslant R$, $t>0$, т.е.

$$
V_{\alpha_{2}}(x, t) \leqslant \delta \Gamma_{\alpha_{2}}(x)-p(x, t), \quad|x| \leqslant R, \quad t>0 .
$$

Применяя в (45) неравенства (34) и (44), будем иметь

$$
V_{\alpha_{2}}(x, t)<\varepsilon \text { при } t \geqslant T(\varepsilon), \quad x \in K,
$$


т.е. доказано, что существует предел (31), равномерньй по $x$ на каждом компакте $K$ в $\mathbb{R}^{N}$.

Теорема 4 доказана.

Рассмотрим задачу Коши

$$
\begin{gathered}
\Delta V_{\alpha}+\left(b(x), \nabla V_{\alpha}\right)+q_{\alpha}(x) V_{\alpha}-V_{\alpha t}=0 \quad \text { в } \mathbb{R}_{+}^{N+1}, \\
V_{\alpha}(x, 0)=\left|u_{0}(x)\right|,
\end{gathered}
$$

где $q_{\alpha}(x)$ определено по формуле $(36,2), b_{k}(x)(k=1, \ldots, N)$ удовлетворяет условиям $\left(\mathrm{B}_{2}\right), u_{0}(x)$ удовлетворяет условию $(81,1)$.

Применением принципа максимума легко устанавливается неравенство

$$
\left|u_{\alpha}(x, t)\right| \leqslant V_{\alpha}(x, t),
$$

где $V_{\alpha}(x, t)$ - решение задачи $(46),(48)$, а $u(x, t)$ - решение задачи $(77,1),(78,1)$ с $L=\Delta$.

Точно так же, как в лемме 24 , доказьвается свойство монотонного убывания по $\alpha$ решений задачи Коши (46), (47)

$$
V_{\alpha_{1}}(x, t) \leqslant V_{\alpha_{2}}(x, t), \quad \alpha_{1}>\alpha_{2} .
$$

$\mathrm{B} \mathbb{R}^{N}$ рассмотрим неравенство

$$
\Delta \Gamma_{\alpha}+\left(b(x), \nabla \Gamma_{\alpha}\right)+q_{\alpha}(x) \Gamma_{\alpha} \leqslant 0,
$$

где коэффициент $q_{\alpha}$ определен в $(36,2)$.

Лемма 26. Для $m>0, B>0 u \alpha_{0}^{2}=m(m+N-2+B)$ существует иисло $l>0$, для которого справедливо неравенство

$$
V_{\alpha_{0}}(x, t) \leqslant l \Gamma_{\alpha_{0}}(x), \quad(x, t) \in \mathbb{R}_{+}^{N+1},
$$

әде $V_{\alpha_{0}}(x, t)$ - решение задачи (46), (48), $\Gamma_{\alpha_{0}}$ - решение неравенства (50) nри $\alpha=\alpha_{0}$.

ДокАЗАТЕЛЬСТво. При $\alpha_{0}^{2}=m(m+N-2+B)$ уравнение $(83,2)$ имеет корни

$$
l_{1}=m>0, \quad l_{2}=2-m-N-B<0,
$$

и функция $(88,2)$ тогда имеет вид

$$
\Gamma_{\alpha_{0}}^{1}(|x|)= \begin{cases}n_{1}|x|^{m}+n_{2}|x|^{2-N-m-B}, & |x|>1, \\ 1, & |x| \leqslant 1,\end{cases}
$$

где

$$
n_{1}=\frac{N+B+m-2}{N+B+2 m-2}, \quad n_{2}=\frac{m}{N+B+2 m-2} .
$$

Далее, рассматривая функцию

$$
z(x, t)=l \Gamma_{\alpha_{0}}(|x|)-V_{\alpha_{0}}(x, t),
$$

точно так же, как в лемме 25, мы докажем неравенство (51).

Лемма 26 доказана.

Неравенства (48), (49) и (51) являются ключевыми для доказательства теоремы 5, и дальнейший ход доказательства теоремы 5 аналогичен доказательству теоремы 4 , и ради краткости не будем это повторять и будем считать, что теорема 5 доказана. 


\section{$\S 4$. О точности результатов п. $4^{\circ} \S 1$}

В работе [33] установлены утверждения, которые показывают точность условий теорем 1 и 2 из $\S 1$ п. $4^{\circ}$, когда коэффициенты $b_{k}$ в уравнении $(77)$ отсутствуют.

Докажем лемму 3 из $\S 1$ п. $4^{\circ}$ при $N=2$.

$\mathrm{B} \mathbb{R}^{2}$ рассмотрим уравнение

$$
\Delta \Gamma+b_{1}(x) \Gamma_{x_{1}}+b_{2}(x) \Gamma_{x_{2}}+c(x) \Gamma=0, \quad x=\left(x_{1}, x_{2}\right) \in \mathbb{R}^{2},
$$

где коэффициенты определены формулами

$$
\begin{aligned}
& c(x)= \begin{cases}-\alpha^{2} & \text { при } r \leqslant 1, \\
0 & \text { при } r>1,\end{cases} \\
& b_{k}(x)= \begin{cases}\frac{x_{k}}{r^{2}} & \text { при } r \geqslant 1, \\
0 & \text { при } r<1 .\end{cases}
\end{aligned}
$$

Будем искать решение уравнения (1) в виде функции

$$
\Gamma=\Gamma(r)>0, \quad \Gamma^{\prime}(r) \geqslant 0, \quad r>0 .
$$

Уравнение (1) с коэффициентами (2), (3) имеет вид

$$
\Gamma_{r r}+\frac{1}{r} \Gamma_{r}-\alpha^{2} \Gamma=0, \quad r<1 .
$$

Рассмотрим решение уравнения (4), удовлетворяюшее условиям

$$
\Gamma(0)=1, \quad \Gamma_{r}(0)=0,
$$

и получим, что это решение имеет вид

$$
\Gamma(r)=I_{0}(\alpha r), \quad r>0,
$$

где $I_{\nu}(x)$ - функция Бесселя порядка $\nu$ мнимого аргумента [91].

При $r>1$ уравнение (1) с коэффициентами (2), (3) имеет вид

$$
\Gamma_{r r}+\frac{2}{r} \Gamma_{r}=0
$$

Рассмотрим решение уравнения (7), удовлетворяющее условиям

$$
\left.\Gamma\right|_{r=1}=I_{0}(\alpha),\left.\quad \Gamma_{r}\right|_{r=1}=\alpha I_{1}(\alpha)
$$

Обшее решение уравнения (7) имеет вид

$$
\Gamma=\frac{C_{1}}{r}+C_{2}, \quad r>1,
$$

где постоянные определяются из (8), и мы получим

$$
\Gamma(r)=-\frac{\alpha}{r} I_{1}(\alpha)+\alpha I_{1}(\alpha)+I_{0}(\alpha) .
$$


Определим начальную функцию $u_{0}(x)$ формулой

$$
u_{0}(x)= \begin{cases}I_{0}(\alpha|x|) & \text { при }|x| \leqslant 1, \\ \Gamma(|x|) & \text { при }|x| \geqslant 1,\end{cases}
$$

где $\Gamma(|x|)$ определено в (9), и рассмотрим задачу Коши

$$
\begin{gathered}
\Delta u+b_{1}(x) u_{x_{1}}+b_{2}(x) u_{x_{2}}+c(x) u-u_{t}=0, \\
\left.u\right|_{t=0}=u_{0}(x)
\end{gathered}
$$

где коэффициенты определены в $(2),(3)$ соответственно, а $u_{0}(x)$ определено формулой (10). Тогда решение (11), (12) имеет вид

$$
u(x, t)=u_{0}(x)>0, \quad t>0 .
$$

Ясно, что $\lim _{t \rightarrow \infty} u(x, t)=u_{0}(x)>0$ и решение задачи Коши $(11),(12)$ не стабилизируется к нулю ни в одной точке $x \in \mathbb{R}^{N}$.

Лемма 3 доказана.

ДокАЗАТЕЛЬСТво ЛЕммы $5 \S 1$ П. $4^{\circ} . \mathrm{B} \mathbb{R}^{N}$ при $N \geqslant 3$ рассмотрим уравнение

$$
\Delta \Gamma+\sum_{k=1}^{N} b_{k}(x) \Gamma_{x_{k}}+c(x) \Gamma=0
$$

где

$$
\begin{gathered}
b_{k}(x)= \begin{cases}\frac{x_{k} m}{|x|^{2-\varepsilon}} & \text { при }|x|>1, m>0, \\
0 & \text { при }|x| \leqslant 1,\end{cases} \\
c(x)= \begin{cases}-\frac{\alpha^{2}}{|x|^{2}} & \text { при }|x|>1, \\
0 & \text { при }|x| \leqslant 1 .\end{cases}
\end{gathered}
$$

Будем искать решение уравнения (14) при $|x|>1$ в виде $\Gamma=\Gamma(r)$, где $\Gamma(r)>0$, $\Gamma^{\prime}(r) \geqslant 0$ при $r>0$. Учитьвая формулы (15), (16), получим, что уравнение (14) можно записать в виде

$$
\Gamma_{r r}+\left(\frac{N-1}{r}+\frac{m}{r^{1-\varepsilon}}\right) \Gamma_{r}-\frac{\alpha^{2}}{r^{2}} \Gamma=0, \quad r>1
$$

Ишем решение уравнения (17), удовлетворяющее условиям

$$
\left.\Gamma\right|_{r=1}=1,\left.\quad \Gamma_{r}\right|_{r=1}=0
$$

Сделаем в (17) замену функшии по формуле

$$
\Gamma(r)=V(r) \exp \left(-\frac{1}{2} \int_{1}^{r} p(\sigma) \mathrm{d} \sigma\right), \quad p(\sigma)=\frac{N-1}{\sigma}+\frac{m}{\sigma^{1-\varepsilon}} .
$$


Получим, что $V(r)$ удовлетворяет уравнению

$$
V^{\prime \prime}-\frac{V}{r^{2-2 \varepsilon}}\left[\frac{m^{2}}{4}+\frac{C_{1}}{r^{\varepsilon}}+\frac{C_{2}}{r^{2 \varepsilon}}\right]=0, \quad r>1,
$$

и условиям

$$
\left.V\right|_{r=1}=1,\left.\quad V^{\prime}\right|_{r=1}=\frac{p(1)}{2}=\frac{N-1+m}{2}
$$

где

$$
C_{1}=\frac{m}{2}(N+\varepsilon-2), \quad C_{2}=\alpha^{2}+\frac{(N-1)(N-3)}{4}, \quad r>0 .
$$

Рассмотрим задачу

$$
\begin{aligned}
& h^{\prime \prime}-\frac{h}{r^{2-2 \varepsilon}}\left[\frac{m^{2}}{4}+\frac{2 C_{1}+2 C_{2}}{r^{\varepsilon}}\right]=0, \\
& \left.h\right|_{r=1}=1,\left.\quad h^{\prime}\right|_{r=1}=\frac{N-1+m}{2} .
\end{aligned}
$$

Легко видеть, что

$$
h(r) \geqslant V(r) \text { при } r \geqslant 1 .
$$

Вводя обозначение

$$
Q(r)=\frac{1}{r^{2-2 \varepsilon}}\left[\frac{m^{2}}{4}+\frac{2 C_{1}+2 C_{2}}{r^{\varepsilon}}\right],
$$

перепишем (22), (23) в виде

$$
h^{\prime \prime}-h Q(r)=0,\left.\quad h\right|_{r=1}=1,\left.\quad h^{\prime}\right|_{r=1}=\frac{N-1+m}{2} .
$$

Вводя в рассмотрение функцию

$$
\alpha_{1}(r)=\frac{1}{8} \frac{Q^{\prime \prime}(r)}{Q^{3 / 2}(r)}-\frac{5}{32} \frac{\left(Q^{\prime}(r)\right)^{2}}{Q^{5 / 2}(r)},
$$

легко показать, что

$$
\int_{1}^{\infty}\left|\alpha_{1}(r)\right| \mathrm{d} r<1
$$

и, кроме того, $Q(r)>0$ при $r>1, Q^{\prime \prime}(r)$ непрерьвна при $r>1$ и

$$
\lim _{r \rightarrow \infty} \frac{Q^{\prime}(r)}{Q^{3 / 2}(r)}=0
$$

Следовательно, вьполнены все условия известной теоремы [92] о (В.К.Б.) асимптотике решений уравнения (25), т.е. сушествуют два линейно-независимых решения $y_{1,2}(r)$ уравнения (25) и

$$
\begin{aligned}
& y_{1}(r)=[Q(r)]^{-1 / 4} \exp \left\{\int_{1}^{r} \sqrt{Q(\sigma)} \mathrm{d} \sigma\right\}\left(1+\varepsilon_{1}(r)\right), \\
& y_{2}(r)=[Q(r)]^{-1 / 4} \exp \left\{-\int_{1}^{r} \sqrt{Q(\sigma)} \mathrm{d} \sigma\right\}\left(1+\varepsilon_{2}(r)\right),
\end{aligned}
$$


где $\varepsilon_{1,2}(r) \rightarrow 0$ при $r \rightarrow \infty$, и эти асимптотические формулы можно дифференцировать по $r$ :

$$
\begin{gathered}
y_{1,2}^{\prime}(r)= \pm[Q(r)]^{1 / 2} \exp \left\{ \pm \int_{1}^{r} \sqrt{Q(\sigma)} \mathrm{d} \sigma\right\}\left(1+\varepsilon_{3,4}(r)\right), \\
\varepsilon_{3,4}(r) \rightarrow 0, \quad r \rightarrow \infty .
\end{gathered}
$$

С помощью решений $y_{1}(r), y_{2}(r)$, имеющих асимптотику $(26),(27)$, построим решение задачи $(22),(23)$ и определим главньй член асимптотики при $r \rightarrow \infty$ :

$$
\begin{aligned}
& h(r)=C_{1} y_{1}(r)+C_{2} y_{2}(r), \\
& C_{1} y_{1}(1)+C_{2} y_{2}(1)=1, \\
& C_{1} y_{1}^{\prime}(1)+C_{2} y_{2}^{\prime}(1)=\frac{N+m-1}{2} .
\end{aligned}
$$

В итоге получим, что

$$
h(r)=C \sqrt{\frac{2}{m}} r^{(1-\varepsilon) / 2} \exp \left\{\frac{m}{2 \varepsilon}\left[r^{\varepsilon}-1\right]\right\}(1+O(1)) \text { при } r \rightarrow \infty .
$$

Используя (19), (24), (29), будем иметь

$$
\begin{aligned}
0<\Gamma(r) & =V(r) \frac{1}{r^{(N-1) / 2}} \exp \left\{-\frac{m}{2 \varepsilon}\left[r^{\varepsilon}-1\right]\right\} \\
& \leqslant \frac{h(r)}{r^{(N-1) / 2}} \exp \left\{-\frac{m}{2 \varepsilon}\left[r^{\varepsilon}-1\right]\right\},
\end{aligned}
$$

и так как для $h(r)$ имеет место (29), то, очевидно, получим, что правая часть (30) стремится к нулю при $r \rightarrow \infty$, т.е.

$$
\lim _{r \rightarrow \infty} \Gamma(r)=0
$$

Определим в задаче Коши

$$
\begin{gathered}
\Delta u+\sum_{k=1}^{N} b_{k}(x) u_{x_{k}}+c(x) u-u_{t}=0 \quad \text { в } \mathbb{R}_{+}^{N+1}, \\
u(x, 0)=u_{0}(x), \quad x \in \mathbb{R}^{N}
\end{gathered}
$$

коэффициенты по формулам (15), (16), а начальную функцию $u_{0}(x)$ - формулой

$$
u_{0}(x)= \begin{cases}\Gamma(|x|) & \text { при }|x| \geqslant 1, \\ 1 & \text { при }|x| \leqslant 1,\end{cases}
$$

где $\Gamma(r)$ - ограниченное (в силу (31)) решение задачи (17), (18). Ясно, что решение задачи $(32),(33)$ имеет вид $u(x, t)=u_{0}(x)>0$ и, следовательно, $\lim _{t \rightarrow \infty} u(x, t)=$ $u_{0}(x), x \in \mathbb{R}^{N}$.

Таким образом, условия $\left(\mathrm{B}_{2}\right)$ в теореме $2 \S 1$ п. $4^{\circ}$ являются точными.

Лемма 5 доказана. 
ДокАЗАТЕльство леммы $7 \S 1$ П. $4^{\circ}$. Рассмотрим в $\mathbb{R}^{N}$ эллиптическое уравнение $(14)$, в котором $b_{k}(x)$ определим формулами

$$
b_{k}(x)= \begin{cases}\frac{B x_{k}}{|x|^{2}} & \text { при }|x|>2, \\ 0 & \text { при }|x| \leqslant 2,\end{cases}
$$

а коэффищиент $c(x)$ определим формулой

$$
c(x)= \begin{cases}-\frac{\alpha^{2}}{|x|^{2}(\ln |x|)^{s}} & \text { при }|x| \geqslant 2, s>1, \\ 0 & \text { при }|x|<2 .\end{cases}
$$

При $r>1$ уравнение (14) тогда имеет вид

$$
\Gamma_{r r}+\frac{N-1+B}{r} \Gamma_{r}-\frac{\alpha^{2} \Gamma}{r^{2}(\ln r)^{s}}=0, \quad r>2 .
$$

При $s>1$ ищем ограниченное при $r>2$ решение уравнения (37), удовлетворяюшее условиям

$$
\left.\Gamma\right|_{r=2}=1,\left.\quad \Gamma_{r}\right|_{r=2}=0 \text {. }
$$

Сделаем в (37) замены $t=r^{N+B-2}, \Gamma=V(t) / t$. После очевидных преобразований получим

$$
\begin{gathered}
\Gamma_{t t}+\frac{2}{t} \Gamma_{t}-\frac{\alpha^{2}}{(N+B-2)^{s+2} t^{2}(\ln t)^{s}} \Gamma=0, \quad t>2, \\
\left.\Gamma\right|_{t=2^{N+B-2}}=1,\left.\quad \Gamma_{t}\right|_{t=2^{N+B-2}}=0 .
\end{gathered}
$$

Так как $\Gamma=V(t) / t$, то для функции $V(t)$ после подстановки выражений

$$
\Gamma_{t}=\frac{V_{t}}{t}-\frac{V}{t^{2}}, \quad \Gamma_{t t}=\frac{1}{t} V_{t t}-\frac{2}{t} V_{t}+\frac{2 V}{t^{3}}
$$

в (39), (40) получим задачу

$$
\begin{gathered}
V_{t t}-\frac{\alpha^{2}}{(N+B-2)^{2+s}} \frac{V}{t^{2}(\ln t)^{s}}=0 \\
\left.V\right|_{t=2^{N+B-2}}=2^{N+B-2},\left.\quad V_{t}\right|_{t=2^{N+B-2}}=1 .
\end{gathered}
$$

Очевидно, что

$$
\int^{\infty} \frac{t}{t^{2}(\ln t)^{s}} \mathrm{~d} t<\infty \text { при } s>1 .
$$

Следовательно, мы можем применить к $V(t)$ теорему 5 из [93; с. 135], согласно которой если для коэффищиента $Q(t)$ в уравнении

$$
V^{\prime \prime}+Q(t) V=0
$$


сходится интеграл

$$
\int^{\infty} t(Q(t)) \mathrm{d} t<\infty
$$

то любое решение $V(t) \geqslant 0$ этого уравнения имеет предел

$$
V(t) \sim d_{0}+d_{1} t, \quad t \rightarrow \infty
$$

Следовательно,

$$
\Gamma(r) \sim d_{1}+\frac{d_{0}}{r^{N+B-2}} \text { при } r \rightarrow \infty .
$$

Поэтому сушествует предел

$$
\lim _{r \rightarrow \infty} \Gamma(r)=d_{1}<\infty .
$$

Рассмотрим задачу Коши (32), (33), в которой коэффищиенты определены по формулам $(35),(36)$, и положим, что $u_{0}(x)$ определена по формуле $(34)$, в которой $\Gamma(|x|)$ обозначает решение задачи $(37),(38)$. Тогда получим, что $\lim _{t \rightarrow \infty} u(x, t)=u_{0}(x)>0$ при всех $x>0$.

Лемма 7 доказана.

Автор выражает глубокую благодарность академику В.А. Ильину, академику А.М. Ильину и профессору В.А. Кондратьеву за внимание к работе и ряд ценных советов.

\section{СПИСОК ЛИТЕРАТУРЫ}

[1] С. Н. Бернштейн. Ограничение модулей последовательных производных решений уравнений параболического типа // Докл. АН СССР. 1938. Т. 18. №7. С. 385-388.

[2] I. Petrowsky. Zur ersten Randwertaufgabe der Wärmeleitungsgleichung // Compositio Math. 1935. V. 1. № 3. P. 383-419.

[3] A. Tychonoff. Théorèmes d'unicité pour l'équation dela chaleur // Матем. сб. 1935. Т. 42. № 2. C. $199-216$.

[4] М. И. Вишик. Задача Коши для уравнений с операторными коэффициентами, смешанная краевая задача для систем дифференциальных уравнений и приближенный метод их решения // Матем. сб. 1956. Т. 39. № 1. С. 51-148.

[5] А. М. Ильин, А.С. Калашников, О.А. Олейник. Линейные уравнения второго порядка параболического типа // УМН. 1962. Т. 17. № 3. С. 3-146.

[6] О. А. Ладыженская. О единственности решени задачи Коши для параболического уравнения // Матем. сб. 1950. Т. 27. № 2. С. 175-184.

[7] В. А. Ильин. О разрешимости смешанных задач для гиперболического и параболического уравнений // УМН. 1960. Т. 15. № 2. С. 97-154.

[8] В. А. Кондратьев. Краевые задачи для параболических уравнений в замкнутых областях // Труды ММО. 1966. Т. 15. С. 400-451.

[9] В.П. Михайлов. Теорема существования и единственности решения одной граничной задачи для параболического уравнения в области с особыми точками на границе // Труды МИАН. 1967. Т. 91. С. 47-58.

[10] С. Д. Эйдельман. Параболические системы. М.: Наука, 1964.

[11] D. G. Aronson, J. Serrin. Local behavior of solutions of quasilinear parabolic equations // Arch. Ration. Mech. Anal. 1967. V. 25. № 2. P. 81-122.

[12] D. G. Aronson. Non-negative solutions of linear parabolic equations // Ann. Scuola Norm. Sup. Pisa (3). 1968. V. 22. № 4. P. 607-694.

[13] D. G. Aronson. Uniqueness of positive weak solutions of second order parabolic equations // Ann. Polon. Math. 1965. V. 16. P. 285-303.

[14] А. Фридман. Уравнения с частными производными параболического типа. М.: Мир, 1965. 
[15] А. Н. Тихонов. Об уравнении теплопроводности для нескольких переменных // Бюлл. МГУ. Матем. мех. 1938. Т. 1. №9. С. 1-49.

[16] А.Н. Колмогоров, И.Г. Петровский, Н.С. Пискунов. Исследование уравнения диффузии, соединенной с возрастанием количества вещества, и его применение к одной биологической проблеме // Бюлл. МГУ. Матем. мех. 1937. Т. 1. № 6. С. 1-26.

[17] А. И. Вольперт. О распространении волн, описываемых нелинейными параболическими уравнениями // И.Г. Петровский. Избранные труды. Дифференциальные уравнения и теория вероятностей. М.: Наука, 1987. С. 333-358.

[18] В. Д. Репников, С. Д. Эйдельман. Необходимые и достаточные условия стабилизации решения задачи Коши для параболического уравнения // Докл. АН СССР. 1966. Т. 167. № 2. C. $298-301$.

[19] В. В. ЖКиков. О стабилизации решений параболических уравнений // Матем. сб. 1977. T. 104. № 4. C. 597-616.

[20] S. Kamin. On stabilization of solution of the Cauchy problem for parabolic equations // Proc. Roy. Soc. Edinburg Sect. A. 1976. V. 76. № 1. P. 43-53.

[21] M. Krzyżański. Sur l'allure asymptotique des solutions d'équation du type parabolique // Bull. Acad. Polon. Sci. Cl. III. 1956. № 4. P. 247-251.

[22] M. Krzyżański. Sur l'allure asymptotique des solutions des problèmes de Fourier relatifs à une équation linéaire parabolique // Atti. Acad. Naz. Lincei. Rend. Cl. Sci. Fis. Mat. Natur. (8). 1960. V. 28. № 1. P. 37-43.

[23] M. Krzyżański. Sur l'allure asymptotique des potentiels de chaleur et de l'intégrale de Fourier-Poisson // Ann. Polon. Math. 1957. V. 8. № 2. P. 288-299.

[24] А. М. Ильин. О поведении решения задачи Коши для параболического уравнения при неограниченном возрастании времени // УМН. 1961. Т. 16. № 2. С. 115-121.

$[25]$ Ф. Х. Мукминов. О равномерной стабилизации решений первой смешанной задачи для параболического уравнения // Матем. сб. 1990. Т. 181. №11. С. 1486-1509.

[26] А.К. Гущин, В.П. Михайлов, Ю.А. Михайлов. О равномерной стабилизации решения второй смешанной задачи для параболического уравнения второго порядка // Матем. сб. 1985 .. Т. 128 . № 2. С. 147-168.

[27] А. К. Гущин. Стабилизация решений второй краевой задачи для параболического уравнения второго порядка // Матем. сб. 1976. Т. 101. № 4. С. 459-499.

[28] А. В. Лежнев. О поведении при больших значениях времени неотрицательных решений второй смешанной задачи для параболического уравнения // Матем. сб. 1986. Т. 129. № 2. C. $186-200$.

[29] В. Д. Репников. О связи двух типов усреднений функции класса $T_{p}\left(\mathbb{R}^{N}\right) / /$ Докл. АН CCCP. 1983. Т. 272. № 4. С. 798-801.

[30] В. Н. Денисов. О стабилизации интеграла Пуассона в классе функций, имеющих степенной рост // Дифференц. уравнения. 1985. Т. 21. № 1. С. 30-40.

[31] Р. З. Хасьминский. Эргодические свойства возвратных диффузионных процессов и стабилизация решений задачи Коши для параболических уравнений // Теория вероятн. и ее примен. 1960. Т. 5. № 2. С. 196-214.

[32] А. М. Ильин, Р.З. Хасьминский. Асимптотическое поведение решений параболических уравнений и эргодическое свойство неоднородных диффузионных процессов // Матем. сб. 1963. Т. 60. №3. С. 368-392.

[33] В.Н. Денисов. О стабилизации решения задачи Коши для параболических уравнений с младшим членом // Дифференц. уравнения. 2003. Т. 39. № 4. С. 506-515.

[34] В.Н. Денисов. О стабилизации решения задачи Коши для параболического уравнения с коэффициентом младшего порядка и с растущей начальной функцией // Труды семинара им. И. Г. Петровского. 2003. №23. С. 127-148.

[35] В.Н. Денисов. О стабилизации решения задачи Коши для параболического уравнения с младшими коэффициентами и растущей начальной функцией // Докл. РАН. 2004. Т. 397. № 4. C. $439-441$.

[36] А. К. Гущин, В.П. Михайлов, А. Л. Муравей. О стабилизации решений нестационарных граничных задач для линейных дифференциальных уравнений в частных производных // Динамика сплошной среды. 1975. № 23. С. 57-90. 
[37] А. К. Гущин. О зависимости поведения при больших значениях времени решения параболического уравнения от данных задачи // Качественная теория дифференциальных уравнений / Под ред. В. М. Матросова. М., 1988. С. 72-82.

[38] В. Н. Денисов, В. Д. Репников. О стабилизации решения задачи Коши для параболических уравнений // Дифференц. уравнения. 1984. Т. 20. №1. С. 20-41.

[39] О. А. Ладыженская, В.А. Солонников, Н. Н. Уральцева. Линейные и квазилинейные уравнения параболического типа. М.: Наука, 1967.

[40] О. А. Ладыженская, Н. Н. Уральцева. Линейные и квазилинейные уравнения эллиптического типа. М.: Наука, 1973.

[41] W. Fulks. A note on the steady state solutions of the heat equation // Proc. Amer. Math. Soc. 1956. V. 7. № 5. P. 766-770.

[42] R. Narasimhan. On the asymptotic stability of solutions of parabolic differential equations // J. Ration. Mech. Anal. 1954. V. 3. № 3. P. 303-313.

[43] З.И. Халилов. Об исследованиях асимптотической устойчивости решений граничных задач для уравнений с частными производными // Докл. АН Азерб. ССР. 1956. Т. 12. № 6. C. $375-378$.

[44] A. Friedman. Convergence of solutions of parabolic equations to steady state // J. Math. Mech. 1959. V. 8. № 1. P. 57-76.

[45] A. Friedman. Asymptotic behavior of solutions of parabolic equations of any order // Acta. Math. 1961. V. 106. № 1-2. P. 1-43.

[46] N. Eklund. Generalized super-solutions of parabolic equations // Trans. Amer. Math. Soc. 1976. V. 220. P. 235-242.

[47] Ю.Н. Черемных. Об асимптотике решений параболических уравнений // Изв. АН СССР. Сер. матем. 1959. Т. 23. № 6. С. 913-924.

[48] А. М. Ильин. Об одном достаточном условии стабилизации решения параболического уравнения // Матем. заметки. 1985. Т. 37. №6. С. 851-856.

[49] P. Besala, P. Fife. The unbounded growth of solutions of linear parabolic differential equations // Ann. Scuola Norm. Sup. Pisa (3). 1966. V. 20. P. 719-732.

[50] N. Meyers, J. Serrin. The exterior Dirichlet problem for second order elliptic partial differential equations // J. Math. Mech. 1960. V. 9. № 4. P. 513-538.

[51] M. Krzyżański. Sur le problème de Dirichlet pour l'équation linéaire du type elliptique dans un domaine non borné // Atti. Accad. Naz. Lincei. Rend. Cl. Sci. Fiz. Mat. Natur. (8). 1948. V. 4. P. 408-416.

[52] S. Simoda, M. Nagumo. Sur la solution bornée de l'équation aux dérivées partielles du type elliptique // Proc. Japan Acad. 1951. V. 27. P. 334-339.

[53] T. Kusano. On bounded solutions of elliptic partial differential equations of the second order // Funkcial. Ekvac. 1965. V. 7. P. 1-13.

[54] В.В. ЖКиков. Асимптотическое поведение и стабилизация решений параболических уравнений второго порядка с младшими членами // Труды ММО. 1983. Т. 46. С. 69-98.

[55] К. В. Валиков. О стабилизации решений задачи Коши для параболических уравнений второго порядка с младшими коэффициентами // Дифференц. уравнения. 1981. Т. 17. № 9. C. $1641-1655$.

[56] Ф.О. Порпер, С. Д. Эйдельман. Теоремы об асимптотической близости решений одномерных параболических уравнений второго порядка // Труды ММО. 1978. Т. 36. C. $85-130$.

[57] А. К. Гущин, В.П. Михайлов. О стабилизации решения задачи Коши для одномерного параболического уравнения // Докл. АН СССР. 1971. Т. 197. № 2. С. 257-260.

[58] К. В. Валиков. Близость решений задач Коши для некоторых параболических уравнений второго порядка // Дифференц. уравнения. 1987. Т. 23. № 4. С. 686-696.

[59] А. Г. Сорокина. О близости решений задачи Коши для параболических уравнений второго порядка // Матем. заметки. 1983. Т. 34. № 1. С. 113-121.

[60] Ф. О. Порпер, С. Д. Эйдельман. Теоремы об асимптотической близости решений многомерных параболических уравнений // УМН. 1980. Т. 35. № 1. С. 211-212.

[61] В. Д. Репников, С. Д. Эйдельман. Новое доказательство теоремы о стабилизации решения задачи Коши для уравнения теплопроводности // Матем. сб. 1967. Т. 73. №1. C. $155-159$. 
[62] Ю. Н. Дрожжкинов. Стабилизация решений обобщенной задачи Коши для ультапараболического уравнения // Изв. АН СССР. Сер. матем. 1969. Т. 33. № 2. С. 368-378.

[63] Ю.Н. Дрожжинов, Б. И. Завьялов. Квазиасимптотика обобщенных функций и тауберовы теоремы в комплексной области // Матем. сб. 1977. Т. 102. № 3. С. 372-390.

[64] В. Д. Репников. О равномерной стабилизации решений задачи Коши для уравнения теплопроводности с полиномиально растущей начальной функцией // Операторные методы в дифференциальных уравнениях. Воронеж: Изд-во ВГУ, 1979. С. 66-71.

[65] В.Н. Денисов. О необходимых условиях равномерной во всем $E^{N}$ стабилизации решения задачи Коши в классе начальных функций, имеющих степенной рост // Докл. АН СССР. 1982. T. 262. № 4. C. $785-786$.

[66] В.Н.Денисов. О необходимом условии равномерной стабилизации решения задачи Коши для уравнения теплопроводности // Докл. АН СССР. 1980. № 6. С. 1310-1312.

[67] В. Н. Денисов. О необходимом условии равномерной стабилизации решения задачи Коши для уравнения теплопроводности // Дифференц. уравнения. 1982. Т. 18. № 2. С. 290-305.

[68] В.Н. Денисов. К вопросу о необходимом условии стабилизации решения задачи Коши для уравнения теплопроводности, равномерной на любом компакте // Дифференц. уравнения. 1982. Т. 18. № 1. С. 7-16.

[69] А. К. Гущин, В. П. Михайлов. О стабилизации решения задачи Коши для параболического уравнения // Дифференц. уравнения. 1971. Т. 7. № 2. С. 297-311.

[70] Е. Б. Сандаков. О стабилизации решения задачи Коши для параболического уравнения высокого порядка // Дифференц. уравнения. 1977. Т. 13. №12. С. 2256-2264.

[71] Е. Б. Сандаков. О стабилизации решения задачи Коши для параболического уравнения высокого порядка с одной пространственной переменной // Дифференц. уравнения. 1975. T. 11. № 5. С. $869-882$.

[72] Ф. О. Порпер, С. Д. Эйдельман. Теоремы о близости решений параболических уравнений и стабилизации решения задачи Коши // Докл. АН СССР. 1975. Т. 221. № 1. С. 32-35.

[73] Ф. О. Порпер, С. Д. Эйдельман. Теоремы об асимптотической близости и стабилизации для решений многомерных параболических уравнений второго порядка // Методы функционального анализа в задачах математической физики / Под ред. Ю. А. Митропольского. Киев: Изд-во Ин-та матем. АН УССР, 1978. С. 81-144.

[74] А. К. Гущин. Об оценках решений второй краевой задачи для параболического уравнения второго порядка // Труды МИАН. 1973. Т. 126. С. 5-45.

[75] A. K. Guščin. On the behaviour as $t \rightarrow \infty$ of solutions of the second mixed problem for a second-order parabolic equation // Appl. Math. Optim. 1980. V. 6. № 2. P. 169-180.

[76] А. К. Гущин. О равномерной стабилизации решений второй смешанной задачи для параболического уравнения // Матем. сб. 1982. Т. 119. № 4. С. 451-508.

[77] Ф.Х. Мукминов. О поведении при $t \rightarrow \infty$ решений первой смешанной задачи для уравнения теплопроводности в неограниченных по пространственным переменным областях // Диффференц. уравнения. 1979. Т. 15. №11. С. 2021-2033.

[78] Ф. Х. Мукминов. Стабилизация решения первой смешанной задачи для параболического уравнения второго порядка // Матем. сб. 1980. Т. 111. №4. С. 503-521.

[79] Ю. К. Герасимов. О поведении решения третьей краевой задачи для параболического уравнения // Вестник Моск. ун-та. Сер. І. Матем. мех. 1962. № 5. С. 34-43.

[80] В.Г. Ушаков. О поведении решений третьей смешанной задачи для параболического уравнения второго порядка при $t \rightarrow \infty / /$ Дифференц. уравнения. 1979. Т. 15 . № 2 . C. $310-320$.

[81] В. Г. Ушаков. Стабилизация решений третьей смешанной задачи для параболического уравнения второго порядка в нецилиндрической области // Матем. сб. 1980. Т. 111. №1. C. $95-115$.

[82] Д. Гилбарг, Н. Трудингер. Эллиптические дифференциальные уравнения с частными производными второго порядка. М.: Наука, 1989.

[83] В.А. Ильин, В.А. Садовничий, Б. Х. Сендов. Математический анализ. Т. 1, 2. М.: Изд-во Моск. ун-та, 2004.

[84] G. Stampacchia. Le problème de Dirichlet pour les équations elliptiques du second ordre à coefficients discontinus // Ann. Inst. Fourier (Grenoble). 1965. V. 15. № 1. P. 189-258. 
[85] D. Gilbarg, J. Serrin. On isolated singularities of solutions of second order elliptic differential equations // J. Anal. Math. 1955/56. V. 4. P. 309-340.

[86] W. Littman, G. Stampacchia, H. F. Weinberger. Regular points for elliptic equations with discontinuous coefficients // Ann. Scuola Norm. Sup. Pisa (3). 1963. V. 17. P. 43-77.

[87] А. Г. Асланян, В. Б. Лидский. О спектре эллиптического уравнения // Матем. заметки. 1970. Т. 7. № 4. С. 495-502.

[88] Т. М. Керимов, В.А. Кондратьев. О спектре эллиптического оператора второго порядка // Матем. заметки. 1976. Т. 20. № 3. С. 351-358.

[89] М. Г. Крейн, М. А. Рутман. Линейные операторы, оставляющие инвариантным конус в пространстве Банаха // УМН. 1948. Т. 3. № 1. С. 3-95.

[90] Qi S. Zhang. Large time behavior of Schrödinger heat kernels and applications // Comm. Math. Phys. 2000. V. 210. № 2. P. 371-398.

[91] Г. Н. Ватсон. Теория бесселевых функций. М.: ИЛ, 1949.

[92] М. В. Федорюк. Асимптотические методы для линейных обыкновенных диффференциальных уравнений. М.: Наука, 1983.

[93] Р. Беллман. Теория устойчивости решений дифференциальных уравнений. М.: Эдиториал УРСС, 2003.

Московский государственньй

Поступила в редакцию университет им. М. В. Ломоносова

20.05 .2005

E-mail: V.Denisov.g23@g23.relcom.ru 